\title{
INFLUENCE OF BOTTOM TOPOGRAPHY ON CROSS-SHELF CIRCULATION FORCED BY TIME DEPENDENT WIND
}

by

\author{
Vladimir I. Osychny \\ B.Sc. Moscow State University, Moscow \\ (1986) \\ Submitted in partial fulfillment of the \\ requirements for the degree of \\ Master of Science \\ at the
}

\begin{tabular}{|c|}
\hline $\begin{array}{c}\text { MARINE } \\
\text { BIOLOGICAL } \\
\text { LABORATORY }\end{array}$ \\
\hline LIBRARY \\
\hline $\begin{array}{c}\text { WOOOS HOLE, MASS. } \\
\text { W. H. O. } .\end{array}$ \\
\hline
\end{tabular}

MASSACHUSETTS INSTITUTE OF TECHNOLOGY

and the

WOODS HOLE OCEANOGRAPHIC INSTITUTION

September 1997

(c) Vladimir I. Osychny 1997

The author hereby grants to MIT and to WHOI permission to reproduce and to distribute copies of this thesis document in whole or in part.

Signature of Author

Joint Program in Physical Oceanography .

Massachusetts Institute of Technology

Woods Hole Oceanographic Institution

August 28, 1997

Certified by ......
$\begin{array}{r}\text { Kenneth H. Brink } \\ \text { Senior Scientist } \\ \text { Thesis Supervisor }\end{array}$

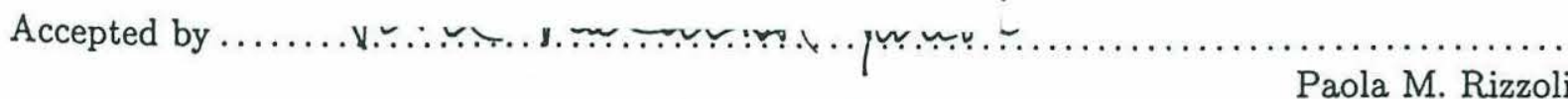

Chairman, Joint Committee for Physical Oceanography

Massachusetts Institute of Technology

Woods Hole Oceanographic Institution 


\title{
Influence of Bottom Topography on Cross-Shelf Circulation Forced by Time Dependent Wind. \\ by

\author{
Vladimir I. Osychny
}

Submitted in partial fulfillment of the requirements for the degree of Master of Science at the Massachusetts Institute of Technology and the Woods Hole Oceanographic

\section{Institution}

\begin{abstract}
A series of numerical experiments is conducted in order to examine the role of topographic irregularities in generation of subinertial cross-channel barotropic currents and to obtain quantitative estimates of the offshore flow amplitude, its ratio to the magnitude of the alongshore currents and the alongshore correlation scale.

A periodic (along the coast) channel with geometry representative of continental margins is considered. Topographic disturbances have multiple alongshore wavenumber contributions, $k_{T}$, and the amplitude proportional to $k_{T}^{-2}$ (except one experiment). The motion is forced by spatially uniform and temporally varying alongshore wind stress, which drives the background current along the channel. The background current adjusts to topographic disturbances and, in particular, Barotropic Shelf Waves (BSW) result. That gives rise to the offshore currents.

The amplitude of the cross-channel flow is shown to increase with $k_{T}$ in the long wave limit (order of $100 \mathrm{~km}$ in the model) and, in contrast, to decrease with $k_{T}$ in the short wave limit (less than $20 \mathrm{~km}$ in the model). As a rule, the strongest response is attained on the intermediate scales where lee waves form most efficiently.

Hart's (1990) quasi-geostrophic solution provided quantitative parameters to explain the scale dependence and helped to interpret the results of simulations meant to examine sensitivity of the cross-channel flow characteristics to variation of the governing parameters. It is shown that the structure of the resonant wave is established by the combination of spatial properties of the bottom bumps, and by the period and amplitude of the fluctuating background current. On the basis of this analysis, it is demonstrated how the BSW dispersion diagram can be used for diagnostic purposes.

The averaged (in time and along the channel) amplitude of subinertial cross-channel currents ranged from negligible values to about $5.5 \mathrm{~cm} / \mathrm{sec}$, indicating that, indeed, rather substantial offshore flow can be generated due to the effect of the topographic irregularities. The alongshore correlation scale was no smaller than about $5 \mathrm{~km}$ and in a few simulations (for instance, with lee-wave-favourable mean wind included) reached $30-50 \mathrm{~km}$.
\end{abstract}

Thesis Supervisor: Kenneth H. Brink

Title: Senior Scientist, Woods Hole Oceanographic Institution 


\section{Table of Contents}

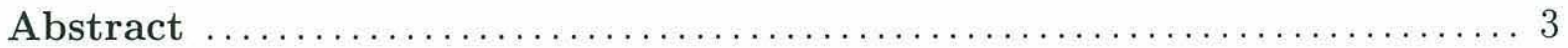

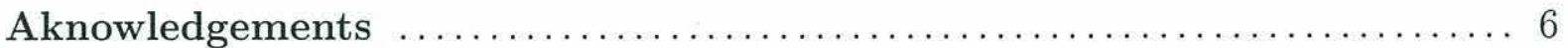

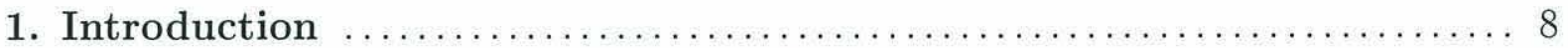

2. The Semi-Spectral Primitive Equation Model (SPEM) ............ 13

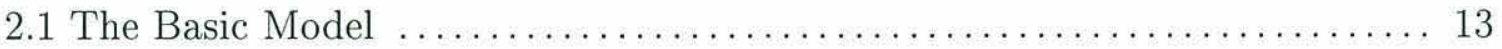

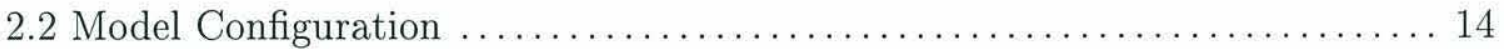

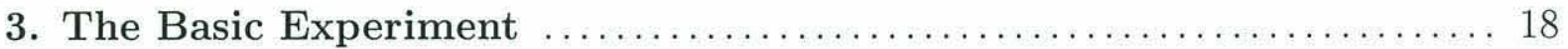

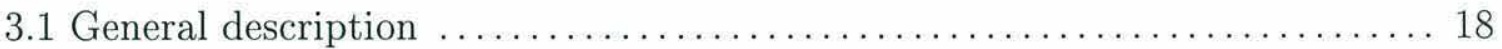

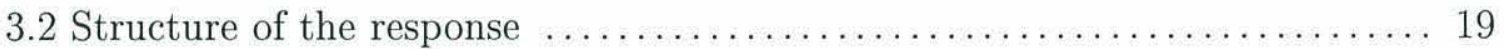

3.3 Generation of the time-mean alongshore flow $\ldots \ldots \ldots \ldots \ldots \ldots \ldots \ldots \ldots 21$

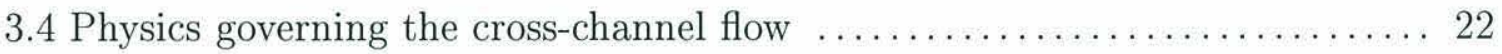

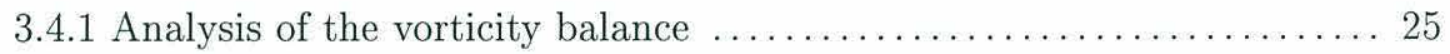

3.4.2 Structure of the cross-channel flow at different alongshore scales ..... 29

3.4.3 Cross-channel flow in the basic experiment ................... 32

4. Dependence of the Offshore Flow Characteristics on the

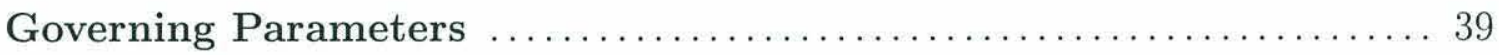

4.1 Dependence on the wind amplitude. (Experiments 24,25) $\ldots \ldots \ldots \ldots \ldots \ldots 39$

4.2 Dependence on the wind period. (Experiments 1-5) $\ldots \ldots \ldots \ldots \ldots \ldots \ldots 41$

4.3 Dependence on friction. (Experiments 16-20) $\ldots \ldots \ldots \ldots \ldots \ldots \ldots \ldots \ldots . \ldots . \ldots . \ldots 2$

4.4 Dependence on amplitude of the bumps. (Experiments 1-15) .......... 43

4.5 Sensitivity to the spectral shape of the bumps

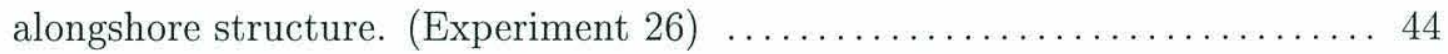

4.6 Sensitivity to a non-monochromatic wind spectrum.

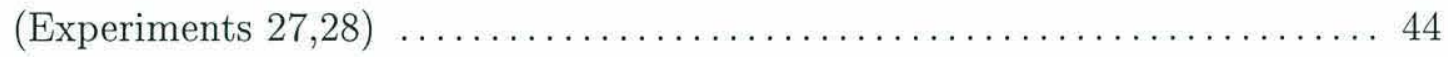

4.7 Addition of non-zero time mean wind. (Experiments 29-30) ........... 45

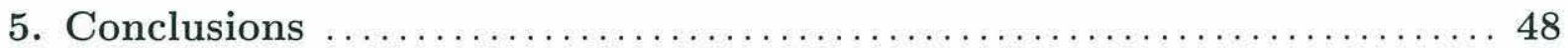

Appendix 1. Governing Parameters in Numerical Experiments . ....... 53

Appendix 2. Influence of a Steady Sheared Background Flow on the BSW Dispersion Characteristics $\ldots \ldots \ldots \ldots \ldots \ldots \ldots \ldots \ldots$

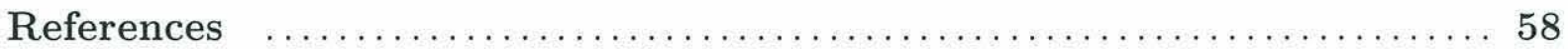

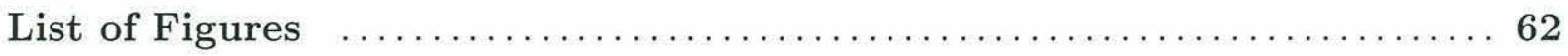




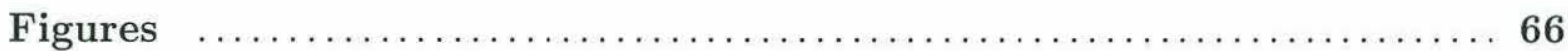




\section{Aknowledgements.}

I am thankful to my advisor, Ken Brink, for his versatile support during the course of my study in the Joint Program, for proposing this research, which was very interesting to me, for his generous patience during my freevolous "hunting for the truth" and for guidance when the hunting sometimes became almost aimless. I appreciate greatly discussions with Dave Chapman, Glenn Flierl and Vitalii Scheremet on the early stages of this research. I am grateful to Kate Hedström for being extremely helpful with all the questions regarding SPEM model and numerous computer problems. The support of Paola Rizzoli and Roger Samelson in finishing this thesis is greatly appreciated. I would like to aknowledge many teachers, whose wisdom and experience define the strength

of the Joint Program, and Education Office, which makes the time in the Program so enjoyable.

I am thankful to my fellow students, especially, to Joe LaCasce, who joyfully shared an office with me for two years.

And, of course, my very special thanks are to my wife, Toma, and my daughter, Dina, for their love, care and understanding in going through these years.

This work was supported by the Office of Naval Research, Coastal Science Program, Grant N00014-95-1-0580. 


\section{Chapter 1}

\section{Introduction.}

Low-frequency (period longer than inertial) alongshore currents in the interior of the water column over continental shelves are relatively successfully predicted in terms of the linear theory of wind forced or free coastal trapped waves, CTWs.(See, for instance, Battisti and Hickey, 1984; Chapman, 1987; Mitchum and Clarke, 1986.) To a large degree, success is achieved due to the fact that most energy of the alongshore velocity component is contained in large scales so that the long wave approximation (Gill and Schumann, 1974) can be made and CTW theory applied.

However, the same models poorly describe the cross-channel flow, which tends to have short alongshore correlation scale, e.g., less than $25 \mathrm{~km}$ according to Winant (1983) and Brink et al. (1994); 4-30 km as pointed out by Dever (1995). Brink et al. (1994) noted that observations in the different shelf zones reveal the amplitude of the offshore velocity component which is smaller but, nevertheless, not negligible (i.e.,approximately, 3 times rather than the order of magnitude smaller, as CTW theory assumes) with respect to the alongshelf current. They hypothesized that the small-scale wind might be responsible for the variability of the cross-shelf currents, although they demonstrated that the wind stress does not possess enough energy at the necessary wavelengths. Similarly, Dever (1995) emphasizes that the wind can explain the offshore flow variability on the scales no shorter than $15-30 \mathrm{~km}$.

Apparently, it is necessary to consider other physical processes leading to the generation of the offshore currents and associated with them small-scale variability. Among possible mechanisms Brink et al. (1994), for example, mention the influences of the alongshore topographic irregularities. It is the goal of the present study to explore the 
detailed physics and to obtain quantitative estimates of the time dependent (period from $1-2$ days to about $2-3$ weeks) cross-shelf flow characteristics (such as, the amplitude, its magnitude relative to the alongshelf velocity component, and the alongshore correlation scale) resulting from the disturbances in the bottom topography.

We use the fact known from observations, that typically the wind stress is a primary energy source for the subinertial flow over the shelf. In order to isolate the effect of the bottom topography, a spatially uniform alongshore wind is considered as the driving mechanism here. Most energy of such a forcing is spent here on the generation of the alongshore currents, which are, in turn, uniform along the coast provided that there are no irregularities in the alongshore direction. Under these conditions the offshore flow is negligible. However, in the presence of topographic disturbances, the flow adjusts to variations in the bottom topography and enhanced cross-shelf currents result.

Bathymetric maps of different shelf zones show alongshore topographic irregularities with many scales of variation. Accordingly, the influences of a continuum of bottom bumps (rather than of an isolated feature) represented by a reasonable alongshore wavenumber spectrum (rather than by single sinusoid, for instance) are examined. The alongshelf scales of the bottom bumps are chosen to range from the order of $10 \mathrm{~km}$ to the order of $100 \mathrm{~km}$.

Thus, in essence, we investigate the interaction of wind-driven alongshore flow with a series of the topographic disturbances. The bottom bumps can have arbitrary amplitude (not necessarily vanishingly small relative to the ambient depth). Also, the low-frequency wind-forced alongshore currents are about $10 \mathrm{~cm} / \mathrm{sec}$ over the shelf. Hence, for the characteristic length $(L \sim 10-100 \mathrm{~km})$, velocity $(V \sim 10 \mathrm{~cm} / \mathrm{sec})$ and time $(T \sim$ 10 days) scales, the ratio of the local to advective accelerations magnitude, $\frac{\varepsilon_{T}}{\varepsilon}=\frac{L}{U T}$, (Pedlosky, 1987), ranges from $10^{-1}$ to $10^{0}$. Therefore, it is expected that non-linearity or, at least, the interaction of the background (alongshore) current with the flow disturbances caused by the topographic bumps, plays a significant role in the dynamics and should be taken into account.

The problem, in general, is rather complicated and in order to make some progress, attention is limited to a purely barotropic case. (The possible restrictions in the applicability of the barotropic analysis to a stratified flow are discussed in Chapter 5.)

There exists an extensive literature addressing issues related to the effect of topo- 
graphic irregularities on barotropic oceanic flow both in general circulation theory and in coastal studies. For instance, Charney and De Vore (1979), Hart (1979), Pedlosky (1981) and Rambaldi and Flierl (1981) consider the possibility of multiple equilibria and blocking resulting from the non-linear interaction of a resonant topographic Rossby wave with steady eastward flow. However, the response in such physical systems is steady or very slowly varying (on a time scale corresponding to the development of nonlinearity). In contrast, Martell and Allen (1979), while studying the generation of barotropic shelf waves (BSW's), which are similar to topographic Rossby waves, by isolated topography, describe properties of the waves excited in a time variable (linearly increasing) background current. Note that time dependence of the forcing is an important feature over the shelves where a considerable amount of energy is supplied to the flow by fluctuating winds with periods ranging from a few days to a few weeks.

Recognizing that, Haidvogel and Brink (1986) used a numerical model to obtain quantitative estimates of the time-mean alongshore flow resulting from topographic drag exerted by zero-mean oscillating alongshelf currents in an environment representative of a continental margin (exponential offshore depth increase, magnitude of friction, wind stress parameters, etc.). The rectified flow originates from differences in the response between two halves of a forcing period: first, when the wind-driven alongshore currents and BSW propagate in the same direction, and second, when they oppose each other and lee waves form producing an extra drag on the flow. Samelson and Allen (1987) and Hart (1990) further develop the problem of alongshore flow rectification by investigating quasi-geostrophic solutions.

Technically, we adopt the Haidvogel and Brink (1986) approach. In particular, the study is based on analysis of results of numerical experiments carried out with different values of the governing parameters. Also, we use a configuration similar to that of Haidvogel and Brink (1986) to describe representative coastal conditions. However, the goal of the present investigation is to explore the physics and to obtain quantitative characteristics of the subinertial cross-shelf currents rather than to examine a rectified alongshore flow. Accordingly, we consider alongshore lengths of the topographic irregularities which are shorter than those used by Haidvogel and Brink (1986): $10-150 \mathrm{~km}$ instead of $30-300 \mathrm{~km}$ (or larger). In addition, in most of the numerical experiments carried out for the purposes of our study, the bottom bumps have multiple scales of 
variation in contrast to monochromatic (single sinusoid) alongshore structure most often used by Haidvogel and Brink (1986). Further, our method of analysis is different.

A brief overview of the numerical model used in this investigation along with a discription of its particular configuration is given in Chapter 2. In Chapter 3, the basic experiment is introduced. First, the major features of the response are outlined. Then, we analyze the generation of the time-mean alongshore currents. The main part of Chapter 3 is devoted to a detailed analysis of the cross-shore flow in the middle of the shelf. A Fourier decomposition of terms in the vorticity balance helps to recognize physical processes governing the response on different alongshore scales. Further, we consider how these processes contribute to the generation of the time-mean and fluctuating components of the cross-shore flow. Then, based on Hart's (1990) quasi-geostrophic solution, it is shown how the BSW dispersion diagram can be used to diagnose the most energetic scale of the cross-shore currents. The results of the basic experiment analysis are exploited in Chapter 4 to explain sensitivity of the offshore flow characteristics to variation of the governing parameters, such as the amplitude and period of the wind stress, the amplitude and the alongshore structure of the bottom bumps, friction, etc. Finally, some conclusions and discussion close the thesis. 


\section{Chapter 2}

\section{The Semi-Spectral Primitive Equation Model (SPEM).}

\subsection{The Basic Model.}

This study is based on the results of numerical experiments conducted using the semispectral primitive equation model (SPEM). The detailed formulation, numerical solution technique and testing of the SPEM has been provided by Haidvogel et al. (1991) and Hedström (1994), so only a brief overview of the model is presented here.

The primitive equations for an incompressible fluid, after making the hydrostatic and Boussinesq approximations, may be written as:

$$
\begin{aligned}
u_{t}+(\vec{u} \cdot \nabla) u-f v & =-\phi_{x}+F^{u}+D^{u}, \\
v_{t}+(\vec{u} \cdot \nabla) v+f u & =-\phi_{y}+F^{v}+D^{v}, \\
0 & =-\phi_{z}-\frac{\rho g}{\rho_{0}} \\
\rho_{t}+(\vec{u} \cdot \nabla) \rho & =F^{\rho}+D^{\rho}, \\
(u)_{x}+(v)_{y}+(w)_{z} & =0,
\end{aligned}
$$

where

$$
\begin{aligned}
(u, v, w) & =(x, y, z) \text { components of velocity vector } \vec{u} \\
f & =\text { Coriolis parameter; } \\
\phi & =\text { perturbation pressure }\left(\frac{p}{\rho_{0}}\right)
\end{aligned}
$$




$$
\begin{aligned}
g & =\text { acceleration of gravity } ; \\
\nabla & =\text { gradient operator; } \\
F^{u}, F^{v}, F^{\rho} & =\text { forcing terms } \\
D^{u}, D^{v}, D^{\rho} & =\text { dissipative terms. }
\end{aligned}
$$

The total density is

$$
\rho_{\text {total }}(x, y, z, t)=\rho_{0}+\rho(x, y, z, t) \text {. }
$$

A rigid lid approximates the surface of the ocean. This eliminates surface gravity waves and enables the use of a streamfunction. The model allows other lateral, bottom and surface boundary conditions to vary, dependent on the physical problem under consideration.

The governing equations are discretized using traditional, centered second-order finite differences in the horizontal. The vertical structure of the variables in the present version of the model is represented as an expansion in a set of Chebyshev polynomials.

The numerical solution for each time step advances through several consecutive procedures. First, the time rate of change of depth-averaged vorticity is determined exploiting the values of all variables at the starting time level. Then, an updated value of vorticity is computed using a leapfrog-trapezoidal time-differencing method. The associated streamfunction field is obtained from the known vorticity distribution as a solution of a generalized elliptic equation with specified boundary conditions. The depth-averaged components of the velocity are found directly from the streamfunction. Internal modes are calculated by time stepping the momentum equations after removing the depthaveraged components. Next, the density field is determined by advancing the density equation in time. Finally, at the new time level, the vertical velocity and the baroclinic pressure field are diagnosed through integrating the continuity equation and hydrostatic relation respectively.

\subsection{Model Configuration.}

We concentrate on studying influences of alongshore irregularities in the bottom topography on purely barotropic flow. For this purpose, the total density is set equal to a constant. 
The continental margin is represented as a cyclic coastal channel of length $L^{x}$ and width $L^{y}$ on an $f$-plane (Fig. 1). Simple straight coastal and offshore walls bound the channel. The background depth increases exponentially offshore and topographic bumps, variable in both cross- and along-channel directions, are superimposed over the background topography:

$$
\begin{aligned}
H(x, y) & =H^{b}(y)+H^{p}(y) \cdot X(x) \\
& =h_{b} \cdot e^{\alpha y}+h_{0} \sin \left(\frac{\pi}{L^{y}} y\right) \cdot \sum_{n=1}^{N} h_{n} \sin \left(\frac{2 n \pi}{L^{x}} x+\varphi_{n}\right) .
\end{aligned}
$$

In all the experiments, the channel width $L^{y}=90 \mathrm{~km}$, the depth at the coast $h^{b}=20 \mathrm{~m}$ and the $e$-folding scale of the background topography $\alpha=5.2 \cdot 10^{-5} \mathrm{~m}^{-1}$. That makes the depth at the offshore boundary equal to approximately $2200 \mathrm{~m}$. The length of the channel $L^{x}=150 \mathrm{~km}$ in most experiments. In fact, only during testing of the model performance were different channel lengths selected.

The flow is forced by a spatially uniform alongshore wind stress which varies sinusoidally in time:

$$
\left\{\begin{array}{l}
\tau^{x}=\tau_{o}+\sum_{m} \tau_{m} \sin \left(\frac{2 \pi}{T_{m}} t+\theta_{m}\right), \\
\tau^{y}=0 .
\end{array}\right.
$$

In most experiments $\tau_{o}$, the time mean wind stress, is zero.

The effect of vertical friction is parameterized according to a linear bottom stress law, in which the bottom resistance coefficient is assumed to be constant. The lateral friction is represented by a biharmonic term and serves only to suppress small-scale computational noise. A comparison of model results for an early stage flow development shows that biharmonic friction (biharmonic friction coefficient $10^{5} \mathrm{~m}^{4} / \mathrm{sec}$ is used) does not appreciably influence the solution at the scales of interest (larger than a few kilometers).

Both wind stress and friction are applied as a body force.

During testing of model performance, the along channel structure of bottom topography consisted of a single sinusoid. However, the main body of experiments has been accomplished with a spectrum of bumps constructed from 15 sinusoids with alongshore wavelength from $10 \mathrm{~km}$ up to $150 \mathrm{~km}$ and randomly selected phases. It has been anticipated that for the surveyed set of model parameters and physical situations, the shortest significant alongshore scale of the excited flow would be close to the shortest along channel wavelength of topographic bumps. Thus, in order to describe wave dynamics properly, 
grid resolution has been set to $\Delta x=0.625 \mathrm{~km}$ ( 16 grid steps per $10 \mathrm{~km}$, which is the shortest wavelength of the bumps) in the alongshore direction and $\Delta y=0.5 \mathrm{~km}$ in offshore direction. Further refinement of spatial resolution did not substantially alter the results discussed below. The time step was of the order of $10 \mathrm{~min}$. and varied depending on the particular experiment to be performed. Overall, if the Courant-Friederichs-Lewy condition

$$
|u|_{\max } \cdot\left(\frac{\Delta t}{\Delta x}\right) \leq 1, \quad|v|_{\max } \cdot\left(\frac{\Delta t}{\Delta y}\right) \leq 1,
$$

has been satisfied, further improvement of the time step would not significantly affect the results.

The vertical resolution was $N^{v}=2$ Chebyshev polynomials analogously to the choice of Haidvogel et al. (1991) in a similar simulation. The results remained depth-independent to machine accuracy as expected because the fluid was homogeneous and the surface (wind) and bottom (friction) stresses were applied as a body force.

The lateral boundary conditions are no normal flow and no slip at the solid walls and periodicity in the along channel direction. The latter, allows description of the channel as an infinite one with the topographic irregularities repeating on a length $L^{x}$.

A typical model experiment starts from a state of rest $(\vec{u}=0)$. The governing equations are integrated in time for 100 days. After $t=60$ days the response in water shallower than $800 \mathrm{~m}$ does not change from one period of forcing to another, so that the results for $t=60-100$ days are used for analysis. 


\section{Chapter 3}

\section{The Basic Experiment.}

\subsection{General description.}

The character of the flow in the model depends on several dynamic and topographic parameters. A number of numerical experiments have been carried out to illuminate the dependence. A description of the model runs is summarized in Appendix 1.

In order to appreciate better the kind of flow we study, a detailed discussion of one simulation is provided first. For that purpose, experiment 3 is chosen (further, we refer to it as to the basic experiment) since the the governing parameters in it are, first of all, typical for the subinertial wind-driven flow over the shelf and, then, 1 ) the wind stress amplitude and period are in the middle of the surveyed range, so that they do not represent low/high frequency or weak/strong forcing limits; 2 ) the amplitude of bumps is the lowest and the friction is the strongest, so that the influences of non-linearity are the weakest and therefore, the dynamics is easier to understand. In later sections, the results of other runs have been compared with those from the basic one.

The bottom topography used in the basic experiment is depicted in Fig. 2 a. As noted in Chapter 2, the alongshore pattern of topographic irregularities is constructed as a sum of 15 sinusoids with wavelengths varying from 10 to $150 \mathrm{~km}$ and phases selected randomly. In the basic experiment, the amplitude associated with each sinusoid is inversely proportional to its wave number, so that the spectrum of the bottom bumps corresponds to $k_{T}^{-2}$ law ( $k_{T}$ is an alongshore wave number of the topographic irregularities). The total rms amplitude of the bottom perturbation at the alongshore section in the middle of the channel in this case is $11.4 \mathrm{~m}$. 
The spatially uniform alongshore wind stress has an amplitude $\tau_{1}=\frac{\hat{\tau}_{1}}{\rho_{o}}=10^{-4} \mathrm{~m}^{2} / \mathrm{sec}^{2}$ (equivalent to $10^{-1} \mathrm{~N} / \mathrm{m}^{2}$ ) and varies sinusoidally in time with a period of 10 days. The bottom resistance coefficient $r=3 \cdot 10^{-4} \mathrm{~m} / \mathrm{sec}^{-1}$, so that the associated $e$-folding frictional time scale, $\left(\frac{H}{r}\right)$, ranges between less than a day and more than 80 days, increasing with depth. Consequently circulation reaches an equilibrium sooner in shallow water.

The flow is forced from a state of rest and computed for 100 days. The results are studied for the last two periods of forcing, $t=80-100$ days.

It is useful for the analysis to express the total flow as the sum of the background current and a perturbation to it. The background flow is a special solution of the barotropic equations:

$$
\begin{aligned}
u_{t}+(\vec{u} \cdot \nabla) u-f v & =-\phi_{x}+\frac{\tau^{x}}{H}-\frac{r}{H} u, \\
v_{t}+(\vec{u} \cdot \nabla) v+f u & =-\phi_{y}-\frac{r}{H} v, \\
(u H)_{x}+(v H)_{y} & =0 .
\end{aligned}
$$

For the case of purely periodic (in time), spatially uniform wind forcing and alongshoreuniform topography, $H^{b}(y)=h_{b} \cdot e^{\alpha y}$, the background solution is:

$$
\left\{\begin{aligned}
U(y, t) & =\frac{\tau^{x}}{H^{b}(y)} \cdot \frac{1}{\left(\frac{r}{H^{b}(y)}\right)^{2}+\omega^{2}} \cdot\left[-\omega \cos (\omega t)+\frac{r}{H^{b}(y)} \sin (\omega t)\right], \\
V & =0
\end{aligned}\right.
$$

where $\omega$ is the wind stress frequency. Then, the perturbation flow is the difference between the total and the background flows and is caused by the existence of topographic irregularities.

\subsection{Structure of the response.}

First, we consider how the flow develops throughout a period and what are the major components of the response, and then we try to understand the physics that governs the essential components of the response.

A sequence of snapshots of the total streamfunction field (Fig. 3) shows the flow in the channel during the last period of forcing. The sinusoidal (in time) background current 
dominates the circulation. The topographic bumps exhibit their influences through the meandering structures in the flow.

Complete history records of both the cross- and along-channel velocity components have been collected at the alongshore sections shown by dotted lines in Fig. 2a. The mean depth at the along-channel section situated approximately $17 \mathrm{~km}$ offshore is about $48.8 \mathrm{~m}$. This section is meant to represent a mid-shelf location. It is the circulation in its vicinity that is of primary concern here.

Evolution of the along-channel flow at the mid-shelf section for the last period of forcing is shown on a time-space diagram (Fig. 4). Again, the resemblance to the background flow is quite close. However, along-channel disturbances are present. Besides that, a difference in the absolute values between maximum and minimum velocities suggests a non-zero time mean flow.

To analyze the perturbation dynamics, it is convenient to use the cross-channel velocity component since it filters out the background current which is directed only along the channel. A time-space diagram of the offshore flow in the deep part of the basin ( $H \approx 2000 \mathrm{~m}$, Fig. 5) indicates consistent propagation of the disturbances in the alongchannel direction. The speed of propagation is of the order of $10 \mathrm{~cm} / \mathrm{sec}$ and is presumably rather weakly affected by the background flow, which is only of the order of $1 \mathrm{~cm} / \mathrm{sec}$ at this depth. The sense of the propagation corresponds to that of Barotropic Shelf Waves (BSW), although it is difficult to identify it with any single mode of BSW at some particular alongshore wavelength.

The diagram of the offshore velocity (Fig.6) for the mid-shelf section reveals a much more complicated structure. For $t=90-96.5$ and $99-100$ days the disturbances travel northward (toward positive $x$ ) at variable speeds. During that time the alongshore flow increases, roughly, from $-15 \mathrm{~cm} / \mathrm{s}$ to its maximum and then diminishes to zero. As it further decreases from $0 \mathrm{~cm} / \mathrm{s}$ to minimum $(t=96.5-99$ days), the disturbances propagate southward. In addition to travelling disturbances, for $t=92.5-99$ days the field acquires a quasi-stationary (non-propagating) component which is still evolving in time locally. A comparison of two diagrams, for the total cross-channel velocity (Fig. 6a) and the time-dependent portion of it (Fig.6b), suggests that a travelling wave dominates during the alongshore flow transition from being maximum southward (negative) to northward (positive), $t=90-93$ and 99-100 days. After the flow reaches a certain positive magni- 
tude (about $15 \mathrm{~cm} / \mathrm{s}$ ), a circulation trapped by topography develops. Further evolution is characterized by the combination of the travelling BSW-like disturbances and those that are quasi-stationary in space.

An example of a time series of the velocity components at a single point $(x=50 \mathrm{~km})$ on the mid-shelf section (Fig. 7) demonstrates temporal changes in the flow more clearly. The flow contains a non-zero time mean, a low frequency component with the period of forcing and higher frequency oscillations with periods of the order of one day. Note that the higher frequency components take place only when the background flow is toward the north, in the direction of free wave phase propagation.

\subsection{Generation of the time-mean alongshore flow.}

The spatial structure of the time-mean velocity components is shown in Fig. 8 and Fig. 11a. The rectified flow is most pronounced over the shelf in the vicinity of the region of the maximum ratio of the topographic perturbation to background topography $\left(H^{\prime}(x, y) / H^{b}(y)\right)$ (see Fig. 2b). The time mean alongshore current is of the order of $1 \mathrm{~cm} / \mathrm{s}$ over the mid-shelf and reaches its maximum of approximately $2.2 \mathrm{~cm} / \mathrm{s}$ near shore over the two steepest northern slopes.

Generation of an alongshore time mean flow in similar conditions has been described by Haidvogel and Brink (1986). It is associated with the topographic drag asymmetry in the oscillatory background current. The asymmetry arises due to excitation of the shelf lee waves only by the southward background current. Then, the current pumps its energy into the lee waves and, thus, slows down. In contrast, shelf waves propagate freely and do not impose any extra drag while the background flow is northward in the direction of the shelf wave propagation. Consequently, the time mean northward flow results after averaging over a period. This process is highlighted on the time-space diagram of the difference between the total alongshore velocity component and the background flow at the mid-shelf section (Fig. 9): the difference is mostly positive during southward background flow $(t=90-91.5$ and $96.5-100$ days $)$.

Haidvogel and Brink (1986) have demonstrated how the residual alongshore flow re- 
lates to topographic irregularities by analyzing the $(x-t)$ averaged $x$-momentum balance:

$$
\frac{\partial}{\partial y} \overline{(H u v)}^{x t}+r \bar{u}^{x t}+{\overline{H \phi_{x}}}^{x t}=0
$$

where $\overline{(~}^{x t}$ denotes averaging in $x$ and $t$. The terms of this equation are shown in Fig. 10a and demonstrate that the primary integrated balance is between topographic stress and bottom friction, similar to the basic experiment of Haidvogel and Brink (1986).

It is interesting to look at how the balance evolves in time (Fig. 10 b,c,d,e). If the averaging is done along the channel only, the momentum balance becomes

$$
{\overline{(H u)_{t}}}^{x}+\frac{\partial}{\partial y} \overline{(H u v)}^{x}+r \bar{u}^{x}+{\overline{H \phi_{x}}}^{x}=0 \text {. }
$$

The local rate of change of momentum is estimated as a residual term. The time evolution of the advective term, $\frac{\partial}{\partial y} \overline{(H u v)}^{x}$, most clearly demonstrates that a non-zero mean flow over a period arises from accumulation of momentum of the same sign during the half of a period when the background current is to the south (negative), $t=90-91.5$ days and $t=96.5-100$ days, and lee waves can form. During the other half, the $\frac{\partial}{\partial y} \overline{(H u v)}^{x}$ term is weaker and its sign alternates, so that an average over this half is negligible.

\subsection{Physics governing the cross-channel flow.}

The structure of the time-mean cross-channel flow (Fig. 11a) is closely reflected in the pattern of its standard deviation (Fig. 11b). Such a resemblance suggests that knowledge of the physics which governs the time-mean offshore flow may be helpful in understanding what determines the gross characteristics (amplitude and the along-channel correlation scale) of the time variable component of the cross-channel flow.

The time-averaged offshore flow seems to be a good indicator of the alongshelf irregularities in bottom topography: positive values (offshore current) are found on the southern slopes of ridges while negative values (onshore current) are located on the northern flanks. The magnitude of the rectified cross channel flow varies from about -3.6 to $+2.2 \mathrm{~cm} / \mathrm{s}$. In terms of vorticity, such a structure of the flow corresponds to the existence of a negative (anticyclonic) anomaly over ridges and a positive (cyclonic) anomaly over canyons. It is apparently associated with the along channel advection of water columns over the irregular bottom topography. 
The effect of such a mechanism is most clearly seen when the ambient topography is flat and the along-channel background current is uniform, so that there is no gradient of the ambient vorticity. Imagine that in these conditions the northward background flow, $U>0$, passes an isolated ridge (Fig. 12a). Then, a fluid column originally at the point $A$ is advected to $B$ and compressed due to topography. In the steady case, in order to preserve potential vorticity, $D_{t}\left(\frac{\varsigma+f}{H}\right) \approx 0$, an anticyclonic (negative) relative vorticity, $\zeta=v_{x}-u_{y}$, is generated over a ridge indicating a presence of the onshore flow over the northern slope and offshore flow over the southern slope of the ridge.

In the present study, the background current is not steady. Instead, it varies sinusoidally in time. For the sake of argument, imagine that the change happens stepwise, so that $U=U_{1}=$ const $>0$ during the first half of a period and $U=U_{2}=-U_{1}$ during the second half of a period. In addition neglect all the transients, i.e., take the period to be long enough for the flow to reach a steady state during each half period. Then observe that the offshore current is not sensitive to the direction of the background flow, i.e., both $U_{1}$ and $U_{2}$ give rise to the same steady cross-channel circulation (Fig. 12a,b), which, therefore, represents offshore flow, averaged over a period. Call this type of adjustment the relative vorticity response for the future use.

However, the presence of cross-channel variations in the background topography substantially changes the physical properties of the system.

First of all, if an ambient current is not too strong or the along-channel length scale of topography is large enough, then the water columns may be advected along isobaths (in essence, around a bump rather than over it) producing negligible (with respect to f) relative vorticity. Hence, the lowest order flow is purely geostrophic flow. Now, consider the above mentioned case of the stepwise evolution of the background current. Then, if the total flow follows isobaths, the cross-channel current at any given location is antisymmetric in time (compare Fig. 12c and 12d). Thus, no cross shelf flow averaged over a period results and its standard deviation is comparable with the amplitude of the cross-channel current during one of the halves of a period. Call this type of adjustment the geostrophic response.

In the more general case, a combination of the relative vorticity change with the lateral advection of ambient vorticity allows barotropic shelf waves. The BSW's, in essence, contain and balance the tendency of flow to follow isobaths and its ability to 
cross them. Thus, physically, BSW's fall in between two steady limits, the short and the long wavelength, represented by the relative vorticity and the geostrophic responses respectively. It has been demonstrated above that the cross-channel flow resulting from the relative vorticity response gives rise to a time-mean component while that originated from the geostrophic response produces a time-fluctuating component. In contrast to the dynamics in the limits, the BSW, in general, is not necessarily a steady feature,i.e., it can either propagate or form lee waves depending on the background flow. Then, how would the BSW's influence the time-mean and the fluctuating components of the cross-channel current?

Again, for simplicity, take the stepwise in time and the uniform across the channel background current. Assume, that this ambient flow generates some resonant BSW and neglect a possible contribution from any other wave. Then, during the first half of a period, when $U>0$, the BSW propagates freely with an increased speed due to Doppler shifting. Subsequently, at any fixed location the currents generated due to the BSW alternate in time and do not contribute substantially to the averaged (over a period) cross-channel flow even if the amplitude of the BSW is amplified due to resonance. During the second half of a period, $U<0$ and a lee wave is formed. Then, at a given location the cross-channel flow is of the same direction throughout the half a period. Its amplitude somewhat increases as the lee wave gains more energy from the background current. Accordingly, a non-zero contribution of the BSW to the mean (over a period) flow comes from the generation of the lee waves while the magnitude of the fluctuating component of the cross-channel flow, which we estimate by taking the standard deviation over a period, depends on the amplitude of the BSW during the both parts of a period.

Obviously, the physics in the basic experiment is even more complicated. The background current varies sinusoidally in time and is sheared across the channel, and several length scales occur due to the complicated structure of the topographic irregularities. Subtle effects of friction and nonlinearity pose several questions, the answers to which are not clear apriori:

1. What is the forcing of perturbation relative vorticity and how does it vary with alongshore scale?

2. What is the physics of the response to forcing at the different length scales and how is it reflected in the structure of the time-mean and the fluctuating components of 
the cross-channel flow?

3. Are there any simple tools to diagnose the dependence of the cross-channel flow characterisics on the parameters of the background current and a structure of topography?

Looking for the answers to these questions, we evaluate a vorticity balance first (3.4.1). Then, based on the results of the numerical experiments in which the along-channel structure of the bumps was relatively simple, we consider how the time mean and the fluctuating components of the cross-channel flow are established at the different $x$-scales (3.4.2). Finally, the cross-channel flow structure in the basic experiment is analysed in details using the quasi-geostrophic solution due to Hart (1990) and the BSW dispersion diagram (3.4.3).

\subsubsection{Analysis of the vorticity balance.}

In order to assess the importance of the competitive physical processes in a generation of the cross-channel velocity, consider the vorticity balance.

After splitting the flow into a variable (in time and across the channel) background current $U$ defined by equation (4) and an along-shore dependent perturbation to it $\vec{u}$, the vorticity equation may be written:

$$
\begin{aligned}
\partial_{t}\left(\frac{\zeta^{\prime}}{H}\right)+\partial_{t}\left(\frac{\zeta}{H}\right)+U \partial_{x}\left(\frac{f}{H}\right)+U \partial_{x}\left(\frac{\zeta}{H}\right)+ & U \partial_{x}\left(\frac{\zeta^{\prime}}{H}\right) \\
+(\vec{u} \cdot \nabla) \frac{f}{H}+(\vec{u} \cdot \nabla) \frac{\zeta}{H}+(\vec{u} \cdot \nabla) \frac{\zeta^{\prime}}{H} & = \\
& \quad-\frac{\tau^{x}}{H} \partial_{y}\left(\frac{1}{H}\right)+\frac{r}{H} \partial_{y}\left(\frac{U}{H}\right)-\frac{r}{H}\left(\nabla \times \frac{\vec{u}}{H}\right)
\end{aligned}
$$

where

$$
\begin{aligned}
\zeta_{\text {total }}=\zeta(y, t)+\zeta^{\prime}(x, y, t) & \text { total relative vorticity; } \\
\zeta(y, t)=-U_{y} & \text { relative vorticity due to the background current; } \\
\zeta^{\prime}(x, y, t)=v_{x}-u_{y} & \text { perturbation relative vorticity due to } \\
& \text { the topographic disturbances in the flow. }
\end{aligned}
$$


All the terms of the equation (3.7) have been calculated for one period of forcing $(t=90-100$ days $)$ at the mid-shelf section, where the standard deviations of the offshore flow and along-channel gradients in topography reach their maxima.

The dominant terms in the averaged (over a period) vorticity balance (Table 1) are the alongshore advection of perturbation relative vorticity by the background current and the advection of background vorticity by flow disturbances:

$$
{\overline{U \partial_{x}\left(\frac{\zeta^{\prime}}{H}\right.}}^{t}+\overline{(\vec{u} \cdot \nabla) \frac{f}{H}}^{t} \approx 0 .
$$

Therefore, generation of the time mean structure of vorticity and, thus, of the time mean offshore currents in the basic experiment can not be explained by only the relative vorticity response (in (3.8) represented by the first term) as it has been proposed in the beginning of section 3.4 .

Table 1. The standard deviation (taken in the along-channel direction) of time mean terms in the vorticity balance (3.7) in $10^{-12} \mathrm{~m}^{-1} \mathrm{sec}^{-2}$.

\begin{tabular}{||c|c|c|c|c|c||}
\hline \hline$\partial_{t}\left(\frac{\zeta^{\prime}}{H}\right)$ & $\partial_{t}\left(\frac{\zeta}{H}\right)$ & $U \partial_{x}\left(\frac{f}{H}\right)$ & $U \partial_{x}\left(\frac{\zeta}{H}\right)$ & $U \partial_{x}\left(\frac{\zeta^{\prime}}{H}\right)$ & \\
\hline 0.00 & 0.00 & 0.00 & 0.18 & 1.51 & \\
\hline \hline$(\vec{u} \cdot \nabla) \frac{f}{H}$ & $(\vec{u} \cdot \nabla) \frac{\zeta}{H}$ & $(\vec{u} \cdot \nabla) \frac{\zeta^{\prime}}{H}$ & $\frac{\tau^{x}}{H} \partial_{y}\left(\frac{1}{H}\right)$ & $\frac{r}{H} \partial_{y}\left(\frac{U}{H}\right)$ & $\frac{r}{H}\left(\nabla \times \frac{\vec{u}}{H}\right)$ \\
\hline 1.31 & 0.03 & 0.26 & 0.00 & 0.00 & 0.46 \\
\hline \hline
\end{tabular}

Evolution of terms throughout a period is represented by their along-channel mean value with a range given by a standard deviation at each time step (Fig.13,14). Thus, Fig. 13 and 14 show the magnitude of terms in the vorticity equation (3.7) for a period of forcing. Their comparison helps to extract the primary time-dependent balance. In order to do that, first, rearrange equation (3.7) by collecting all the terms representing either forcing or damping of perturbation relative vorticity on the right-hand side:

$$
\begin{aligned}
\partial_{t}\left(\frac{\zeta^{\prime}}{H}\right) & +U \partial_{x}\left(\frac{\zeta^{\prime}}{H}\right)+(\vec{u} \cdot \nabla) \frac{f}{H}+(\vec{u} \cdot \nabla) \frac{\zeta}{H}+(\vec{u} \cdot \nabla) \frac{\zeta^{\prime}}{H}= \\
& -\partial_{t}\left(\frac{\zeta}{H}\right)-U \partial_{x}\left(\frac{f}{H}\right)-U \partial_{x}\left(\frac{\zeta}{H}\right)-\frac{\tau^{x}}{H} \partial_{y}\left(\frac{1}{H}\right)+\frac{r}{H} \partial_{y}\left(\frac{U}{H}\right)-\frac{r}{H}\left(\nabla \times \frac{\vec{u}}{H}\right)
\end{aligned}
$$


Now, note that

$$
\partial_{t}\left(\frac{\zeta}{H}\right)=-\frac{\tau^{x}}{H} \partial_{y}\left(\frac{1}{H}\right)+\frac{r}{H} \partial_{y}\left(\frac{U}{H}\right)
$$

essentially represents the background current balance. Indeed, a sum of these terms calculated based on the model results is approximately zero (Fig. 15a). Then, observe that $(\vec{u} \cdot \nabla) \frac{\varsigma}{H}$ is negligible relative to the other left-hand side terms and so is $U \partial_{x}\left(\frac{\zeta}{H}\right)$ in comparison with the other forcing terms (Fig. 13).

Thus, the primary time dependent balance for the perturbations is:

$$
\partial_{t}\left(\frac{\zeta^{\prime}}{H}\right)+U \partial_{x}\left(\frac{\zeta^{\prime}}{H}\right)+(\vec{u} \cdot \nabla) \frac{f}{H} \approx-U \partial_{x}\left(\frac{f}{H}\right)-\left[(\vec{u} \cdot \nabla) \frac{\zeta^{\prime}}{H}+\frac{r}{H}\left(\nabla \times \frac{\vec{u}}{H}\right)\right] .
$$

Nonlinearity, $(\vec{u} \cdot \nabla) \frac{\zeta^{\prime}}{H}$, and the damping of perturbation relative vorticity, $\frac{r}{H}\left(\nabla \times \frac{\vec{u}}{H}\right)$, are not negligible, although, on average, they are less than any other term. Moreover, the standard deviation of their sum (Fig. 15b) is larger than that of either of them taken separately. Comparison with all the other terms in (3.11) taken together (linearized, inviscid balance) depicted in Fig. 15c suggests that nonlinearity acts mainly as an additional damping of vorticity in the system.

The primary balance (3.11), while much simpler than the full vorticity equation (3.7), still describes several distinctive physical mechanisms dependent on the magnitude of parameters involved.

In an attempt to distinguish the dominant processes at the different alongshore scales, Fourier analysis of all terms in the equation (3.7) has been carried out assuming that the balance holds at every length scale. As a result, an amplitude of each term has been calculated at every time step throughout a period of forcing.

Incidentally, a Fourier decomposition confirms that the background current balance (3.10) dominates the vorticity equation at the largest length scale: mode 0 (no alongshore variations). The terms constituting that balance become of secondary importance at all other scales and, moreover, still cancel each other out. Therefore, they are excluded from the further analysis of the perturbation relative vorticity.

Overall, for alongshore modes higher than 0 , evolution of perturbation vorticity is well approximated by equation (3.11). The importance of each term at the different scales may be appreciated through a comparison of their time-mean amplitudes plotted in Figure 16. 
First of all, note that despite the strong dependence of the amplitude of the topographic irregularities on the along-channel length scale (the $x$-spectrum of the bumps is proportional to $\left.k_{T}^{-2}\right)$, the amplitude of the main forcing term, $U \partial_{x}\left(\frac{f}{H}\right)$, does not vary much from one mode to another. In other words, the forcing itself does not depend on the alongshore scale and, subsequently, any variation of the cross-channel flow is an intrinsic feature of the response. The fact that the forcing changes weakly with $k$ could be anticipated from an estimate of the weighting factor, $\left(\frac{1}{H}\right)_{x}$. It might be shown that, if the streamfunction is used, that factor appears as $H_{x}$ in the vorticity equation and at the any given $y=y_{o}$ is:

$$
H_{x}=H^{p}\left(y_{o}\right) \cdot \sum_{n=1}^{N}\left(\frac{2 n \pi}{L^{x}}\right) h_{n} \cos \left(\frac{2 n \pi}{L^{x}} x+\varphi_{n}\right) .
$$

Since the amplitude of each topographic mode, $h_{n}$, is inversely proportional to its number, $n$, the magnitude of this factor does not depend on the alongshore scale:

$$
H_{x} \propto H^{p}\left(y_{o}\right)\left(\frac{2 \pi}{L^{x}}\right) \sum_{n=1}^{N} \cos \left(\frac{2 n \pi}{L^{x}} x+\varphi_{n}\right) .
$$

Thus, the production of vorticity, $U \partial_{x}\left(\frac{f}{H}\right)$, is about the same at all along-channel scales equal to or shorter than $150 \mathrm{~km}$.

According to the results of the calculation, the forcing produces three main kinds of adjustment, depending on the alongshore scale (Fig. 16).

At the long scales, $150 \mathrm{~km}$ and $75 \mathrm{~km}$, the leading term is advection of ambient vorticity by the flow disturbances, $(\vec{u} \cdot \nabla) \frac{f}{H}$. If the relative vorticity part, $\left(\partial_{t}+U \partial_{x}\right)\left(\frac{\zeta^{\prime}}{H}\right)$, were completely neglected, the geostrophic balance, which expresses a tendency of flow to follow isobaths, results in:

$$
U \partial_{x}\left(\frac{f}{H}\right)+(\vec{u} \cdot \nabla) \frac{f}{H}=\left(\vec{u}_{\text {total }} \cdot \nabla\right) \frac{f}{H} \approx 0 .
$$

It is expected then, that at the long scales, the cross-channel flow is close to that which is antisymmetric in time at any given point (a property of the geostrophic response).

Another extreme covers scales of $21.4 \mathrm{~km}$ (mode 7) and shorter on which the change in relative vorticity overwhelms the advection of the background vorticity by the perturbations. In the limit of negligible $(\vec{u} \cdot \nabla) \frac{f}{H}$ such a balance states the relative vorticity response, described in the beginning of the chapter: 


$$
\left(\partial_{t}+\bar{U} \partial_{x}\right)\left(\frac{\zeta^{\prime}}{H}\right) \approx \bar{U} \partial_{x}\left(\frac{f}{H}\right) .
$$

The main feature of this type of adjustment is generation of the time-mean cross-channel flow.

At the intermediate scales, $50-25 \mathrm{~km}$ (modes 3-6), both the relative vorticity and the geostrophic terms are of the similar magnitude. Therefore, it is anticipated that at these scales, BSW dynamics plays the most noticeable role in a determination of a $v$-structure.

\subsubsection{Structure of the cross-channel flow at different alongshore scales.}

The properties of the cross-channel velocity field generated at different scales by the distinct physical mechanisms are demonstrated by results of three numerical experiments in which the topographic bumps are represented by an alongshore structure simpler than in the basic experiment.

Short scale (relative vorticity) response. (Experiment 23.)

An example of the response on the short scale is given for the case in which the topographic perturbation is a single sinusoid of $10 \mathrm{~km}$ wavelength (mode 15). At this scale the evolution of relative vorticity dominates the vorticity balance and the response should not be sensitive to the direction of the background flow. Indeed, the time-mean component, which is negative/positive over the northern/southern flanks of the underwater ridges, overwhelms the total cross-channel velocity field (Fig.17a). Taken over a period and averaged over a channel length the standard deviation of the fluctuating component of $v$ does not exceed $20 \%$ of the mean.

Thus, the short scale response contributes mainly to the mean field and much less to oscillations in time.

Long scale (geostrophic) response. (Experiment 21.)

Results of a numerical experiment in which the bumps are constructed from a sum of mode 1 and 2 (alongshore wavelength 150 and $75 \mathrm{~km}$ ) demonstrate properties of the longscale adjustment. A time-space diagram of $v$ (Fig.18a) shows the geostrophic tendency of flow to follow isobaths and to produce the antisymmetric (in time) cross-channel current, 
manifested through the change of sign of $v$ at a given location between the two halves of a period: when $U>0$ and when $U<0$.

Nevertheless, the $v$-structure is not perfectly antisymmetric.

First, the amplitude of the cross-channel flow is larger when the background current is negative, presumably, due to excitation of lee waves. A Fourier analysis of the timemeans of $v$ taken over two distinct parts of a period, $t=93-95$ days (quasi-stationary regime during no-lee-wave part) and $t=98-100$ days (quasi-stationary regime during lee-wave part), shows (Fig. 18c) that the difference in amplitude between the two parts is considerably larger for the second alongshore mode. Therefore, in this experiment the BSW's of the shorter $(75 \mathrm{~km})$ wavelength are more efficient in producing the cross-channel flow.

Second, the antisymmetry is disturbed by the along-channel phase shift in the $v$ structure between the two parts of a period. (To see the shift, compare, for instance, the alongshore position of extrema of $v$ during the two distinct parts of a period in Figure 18a. Remember that the cross-channel flow switches sign, so that the position of a maximum during $t=93-95$ days should be compared with a minimum during $t=98-100$ days.) The magnitude of the shift may be estimated through finding a lag corresponding to a maximum of the absolute value of the cross-correlation function between the above mentioned time-means of $v$. The correlation coefficient has a maximum absolute value when it is negative, -0.98 , and the mean taken during the lee-wave phase $(t=98-100$ days $)$ is shifted southward by $9.4 \mathrm{~km}$. The origin of the shift is associated with influence of friction as explained by Hart (1990).

Note that both the difference in amplitude and the along-channel shift in $v$ structure produce the non-zero time-averaged offshore flow. In the given case, both factors are larger for the wavelength $75 \mathrm{~km}$. Thus, a contribution of this length scale to the timeaveraged cross-channel flow is stronger than that of $150 \mathrm{~km}$.

The time variable component of the offshore current at long scales results mainly from the antisymmetric (geostrophic) part of the solution.

\section{Intermediate scale response ( $B S W$ dynamics).}

On intermediate scales, the terms representing change of the flow due to generation of relative vorticity and due to advection of the ambient vorticity are of a similar magnitude. To demonstrate what kind of cross-channel flow is established from such a type of 
adjustment, an example with topographic disturbances composed from a sum of modes 4,5 and 6 (alongshore wavelength of $37.5,30$ and $25 \mathrm{~km}$ respectively) is used.

Again, during the no-lee wave phase, the extrema of cross-channel velocity are found over the slopes of the ridges in bottom topography (Fig.19a). Qualitatively, the structure of $v$ is in agreement both with the geostrophic tendency of the flow to follow isobaths and with an offshore flow originating from the relative vorticity response (when $U>0$ both mechanisms give rise to a $v$ of the same direction).

During the lee-wave part of a period, the offshore velocity has a larger amplitude (Fig.19c). The largest difference in amplitude between the two means is for mode 4 (37.5 $\mathrm{km}$ wavelength). Thus, the BSW's produce the cross-channel flow most efficiently on the scale $37.5 \mathrm{~km}$, which is the longest one in this experiment. (In contrast to the long scale response, where the largest contribution came from the shortest wavelength.)

In addition, the whole structure is shifted southward in comparison with the no-lee wave part of a period. The correlation coefficient between the time-means taken during $t=93-95$ days and $t=98-100$ days is -0.98 with the lee-wave part being shifted southward by $11.3 \mathrm{~km}$. Note that such a distance shift, which is comparable to that observed in the long scale response, translates into an appreciable (in contrast to the long scale response) phase shift due to decreased wavelength.

Both the mean and fluctuating component of the cross-channel circulation at the intermediate scales have significant amplitude (Fig.20). Besides, the resemblance of their alongshore structure with the $v$-field during the lee-wave part suggests that a dominant role in their determination belongs to the considerably stronger (2-3 times) amplitude of $v$ at that stage relative to the no-lee wave part of a period. That is, the standard deviation of $v$ calculated for a whole period is dominated by the more intensive increase of the offshore flow amplitude during the lee-wave part. Again, this is in contrast to the long scale response, where the fluctuating component is produced mainly by the change of the cross-channel flow direction (rather than change of its amplitude), i.e., by the antisymmetric in time (geostrophic) part of the solution.

The calculation of the mean (over a period) autocorrelation function of the fluctuating component of $v$ further supports the dominant importance of lee-waves in the crosschannel velocity structure. The autocorrelation function has a significant maximum of 0.62 at $37.5 \mathrm{~km}$ lag which we recognize as a correlation scale of the oscillating part of the 
offshore current. Note, that this length coincides with the most energetic scale during the lee wave phase of a period.

Also, note that when the topographic bumps have a more complicated alongshore structure, the maximum of the autocorrelation function is not so well established and a definition of the alongshore correlation scale becomes obscure. For a comparison of responses between different experiments we adopt an e-folding scale of the autocorrelation function as a correlation scale.

\subsubsection{Cross-channel flow in the basic experiment.}

So far we have recognized physically distinct alongshore scales and analyzed how the timemean and fluctuating components of the cross-channel flow are established at those scales taken separately. Next, the offshore velocity is considered in the basic experiment, in which the topographic perturbation is represented by a mix of modes 1-15 and, therefore, different mechanisms act simultaniously. Then, a discussion of the quasi-geostrophic solution due to Hart (1990) is provided and, based on Hart's theory, the structure of the cross-channel flow in the basic experiment is interpreted.

A Fourier analysis of the cross-channel flow in the basic experiment shows a different spectral shape during the no-lee-wave and the lee-wave parts of a period (Fig.21a). When $U>0$ (no-lee-wave phase, $t=91.5-96.5$ days) energy of the cross-channel flow monotonically increases as alongshore wavelength increases (ignore the amplified power at intermediate scales $50-25 \mathrm{~km}$ (mode $3-6$ ) during $t=91.5-93$ days as a remnant of the lee-wave phase activity). Throughout the lee-wave part $(t=90-91.5$ and $t=96.5-100$ days) the offshore velocity spectrum has a maximum at intermediate scales. Overall, most of the total variance of $v$ is concentrated in the modes 1-6 $(150-25 \mathrm{~km})$. Observe that at all these scales the amplitude starts to increase as $U$ becomes negative. From the beginning $(t=96.5$ days $)$ it happens more powerfully at $50 \mathrm{~km}$ wavelength (mode 3 ). Only during $t=90-92.5$ days the next scale, $37.5 \mathrm{~km}$, becomes more energetic. Thus, the lee waves of alongshore scales from $150 \mathrm{~km}$ to $25 \mathrm{~km}$ are excited simultaneously although with a different efficiency in the sense of generating a cross-channel flow.

It follows from the discussion in the previous section that the long-scale modes 1 
and 2 provide most of their energy to the fluctuating component of $v$, while modes 3-6 contribute both to the fluctuating and time mean parts of the cross-channel flow. Figure 21c shows that, in general, the ratio of the energy of the oscillating component of $v$ to the total power of $v$ increases with along-channel scale. Nevertheless, the most energetic absolute contribution to the total offshore velocity, to its fluctuating component as well as to the time mean over a period, is supplied by the alongshore mode $3(50 \mathrm{~km})$ (Fig. 21b), which is the most energetic wavelength during the lee-wave phase of a period.

Is it then possible to recognize the most energetic scale a priori, i.e., based on the known background current and a structure of the topographic irregularities ? And why does the spectrum of the cross-channel flow have the shape described above ?

Here we rely on the analysis of a similar quasi-geostrophic problem due to Hart (1990). He considered an equivalent $\beta$-plane flow over irregular bottom topography forced by the time-periodic axisymmetric background current in a cylinder. Hart's solution is adopted for a case of the straight channel with a linearly increasing (in $y$ ) ambient bottom topography. The background current is sinusoidal in time, but in contrast to the basic case, is uniform across the channel.

Then, the quasi-geostrophic vorticity equation can be written in non-dimensional form as (Pedlosky, 1987)

$$
\left[\partial_{t}+U(t) \partial_{x}\right] \nabla^{2} \phi+\beta \phi_{x}+\epsilon J\left(\phi, \nabla^{2} \phi\right)+J\left(\phi, \eta_{B}\right)=-\hat{r} \nabla^{2} \phi-\frac{U}{\epsilon} \partial_{x} \eta_{B}
$$

where $\psi=-U_{o} y \sin (t)+\epsilon \phi$ is the geostrophic streamfunction such that $\psi_{y}=-u_{\text {total }}$, $\psi_{x}=v$; the time scale is advective time $U_{o} / L, U_{o}$ is an amplitude of the velocity and $L$ is a characteristic horizontal length; $\beta$ is the ambient vorticity gradient represented by the cross-channel gradient of the background topography and scaled by a typical value of the relative vorticity gradient $U_{o} / L^{2}$; friction acts through an Ekman layer of typical thickness $\delta_{E}: \hat{r}=\frac{1}{R o} \frac{\delta_{E}}{D}$, while $D$ is a characteristic depth of the channel; $J$ is a Jacobian operator. The topographic perturbation has the same structure as in the basic experiment and its magnitude is chosen to be of the order of $\epsilon: \eta_{B}=\epsilon h_{o} \cos (k x) \sin (l y)$.

It has been noticed in the previous sections (dicussion after equation (3.11)) that nonlinearity in the basic experiment acts essentially as an additional friction. Therefore, attention is limited to the linearized dynamics. Formally, the terms of the order of $\epsilon^{2}$ are 
neglected in (3.12) and the vorticity equation becomes:

$$
\left[\partial_{t}+U_{o} \sin (t) \partial_{x}\right] \nabla^{2} \phi+\beta \phi_{x}=-\hat{r} \nabla^{2} \phi-U_{o} \sin (t) h_{o} \partial_{x}[\cos (k x) \sin (l y)]
$$

Similar to Hart, look for solution

$$
\phi=R e\left[F(t) \sin (l y) e^{i k x}\right]
$$

Then, the problem is to find a complex amplitude $F$ from the first order equation:

$$
\frac{d F}{d t}+\hat{r} F-i \frac{\beta k}{K^{2}} F\left[1-\frac{U_{o}}{\beta / K^{2}} \sin (t)\right]=-i \frac{U_{o} k}{K^{2}} h_{o} \sin (t)
$$

where $K^{2}=k^{2}+l^{2}$ is a total wavenumber. Note also, that $\omega_{R}=\frac{\beta k}{K^{2}}$ and $c_{R}=\frac{\beta}{K^{2}}$ are the frequency and phase speed of the topographic Rossby wave.

After the decaying component, necessary to satisfy the initial conditions, is dropped, the solution is

$$
F(t)=-\frac{2 \frac{U_{o} k}{K^{2}}}{\omega_{R} \frac{U_{o}}{c_{R}}} h_{o} e^{i \omega_{R} \frac{U_{o}}{c_{R}} \cos (t)} \sum_{\nu}^{\infty} \nu e^{-i \pi \nu / 2} J_{\nu}\left(\omega_{R} \frac{U_{o}}{c_{R}}\right) \frac{\gamma \sin (\nu t)-\nu \cos (\nu t)}{\nu^{2}+\gamma^{2}},
$$

where $\gamma=\hat{r}-i \omega_{R}$ and $J_{\nu}$ is a Bessel function of order $\nu$. In general, the solution exhibits quite a complicated time evolution and the reader interested in all the details is referred to Hart (1990). We concentrate on several features important for us and,first of all, on evaluation of the dependence of amplitude of $v$ on the alongshore wavenumber $k$.

The largest (resonant) amplitude of $F$ occurs for integer values of $\omega_{R}$, i.e., when the frequency of the excited topographic Rossby wave corresponds to one of harmonics of the fundamental (forcing) frequency. The response is strongest at the fundamental frequency and somewhat diminishes for the higher harmonics. In addition, the amplitude of $F$ depends also on the ratio $U_{o} / c_{R}$, which would determine a resonance if the background flow were steady.

At the resonant frequency, $\nu=\omega_{R}=$ integer number, the amplitude of $F$ varies as

$$
|F| \sim \frac{\frac{U_{o} k}{K^{2}}}{\omega_{R} \frac{U_{o}}{c_{R}}} h_{o} \cdot J_{\nu}\left(\omega_{R} \frac{U_{o}}{c_{R}}\right)=\frac{h_{o}}{K^{2}} J_{\nu}\left(U_{o} k\right) .
$$

If $h_{o}=\hat{h}_{o} / k$ as it is in the basic experiment, then the amplitude of the cross-channel velocity changes as

$$
|v| \sim k F \sim \frac{\hat{h}_{o}}{K^{2}} J_{\nu}\left(U_{o} k\right)
$$


For long alongshore wavelengths (larger than the cross-channel length scale, $k / l<1$ ), $K^{2} \sim l^{2}$, so that:

$$
|v| \sim J_{\nu}\left(U_{o} k\right) .
$$

Note that $J_{\nu}$ grows monotonically for argument less than 1 . Thus, in the long scale limit, the amplitude of the cross-channel flow increases with wavenumber. Physically, in this limit, flow follows isobaths and at the same $U_{o}$ possesses a larger cross-channel component for larger curvature of topography, i.e., for shorter alongshore length scale.

In contrast, at short along-channel lengths (such that $k^{2} / l^{2} \gg 1$ ):

$$
|v| \sim \frac{\hat{h}_{o}}{k^{2}}
$$

Hence, in this limit, the amplitude of the cross-channel velocity diminishes with $k$. That happens due to the fact that, as the alongshore scale of the topographic perturbation decreases, the water columns become advected over the bumps too quickly, so that they do not have enough time to "feel" the variation in bottom topography.

Note that in the amplitude equation (3.14), these two limits result from consideration of the ratio $U_{o} / c_{R}$ : in the short wave limit (relative vorticity response) $U_{o} / c_{R} \gg 1$ and advection of relative vorticity dominates; in the long wave limit (geostrophic response) $U_{o} / c_{R} \ll 1$ and advection of the background vorticity by the perturbation flow prevails. The intermediate scales (BSW response) are those for which $U_{o} / c_{R} \sim 1$. Since the dynamics at the intermediate scales contains the features of both limits, namely, $|v|$ increases with $k$ at long lengths and decreases at short scales, it is concluded that the maximum of the cross-channel amplitude occurs on the intermediate scales. For instance, Hart (1990) shows that the largest amplitude of the time-averaged solution, which is directly related to the generation of lee waves, occurs at the resonant frequencies and at $U_{o} / c_{R}=1$ (see Fig. 3 and 4 of Hart, 1990). (Mathematically, one should find a maximum of (3.16).) Note, that qualitatively, a dependence of $|v|$ on $k$ described above is consistent with the time mean spectrum of the cross-channel flow in the basic experiment.

Physically, the whole situation may be outlined as follows. The topographic Rossby waves are excited by the oscillating (in time) background current at alongshore wavenumbers determined by the bottom bumps and at different frequencies, preferentially, at those corresponding to harmonics of the fundamental (forcing) period. Consequently, the excited waves have different phase speeds and are advected by the background flow with 
different velocities. The largest amplitude is obtained by the waves which have $U_{o} / c_{R}$ of the order of 1 , since they remain stationary when the background current 1) is the most powerful comparatively to other parts of a period (more energy available to transfer to the wave), and 2) retains this critical value for the longest duration (time derivative of the sinusoidal-in-time background current is the lowest, when the flow is close to a maximum amplitude).

How does this discussion apply to the results of the basic experiment?

Consider a BSW dispersion diagram (Fig. 22). It has been shown that parameters of the excited waves are controlled by the combination of 1) spatial structure of the topographic irregularities, and 2) by the amplitude and frequency of the background flow. In the basic experiment, the $x$-structure of the bumps coincides with that of the BSW's, so that the BSW's are generated at the alongshore wavenumbers of the corresponding topographic disturbances. The $y$-structure of bumps, $\sin \left(\frac{\pi}{L^{y}} y\right)$, favors the excitation of the first cross-channel mode of the BSW, $e^{\frac{\alpha}{2} y} \sin \left(\frac{\pi}{L^{y}} y\right)$. The period of forcing is 10 days, so that its subsequent harmonics are $5,3.3,2.5,2, \ldots$ days. Note that the resonant harmonics are more densely distributed in the upper part of the dispersion diagram. Consequently, the set of the resonant harmonics is richer (more resonant frequencies available at particular $k$ ) for the along-channel scales longer than $30 \mathrm{~km}$.

The last important parameter is the ratio $U_{o} / c_{R}$. We take $c_{R}$ to be a phase speed of the first cross-channel mode BSW. A choice of $U_{o}$ is not well determined since in contrast to Hart's problem, where the background current is uniform across the channel, $U$ is $y$-sheared in the basic experiment. The amplitude of $U=U_{o}$ varies across the channel from about $0 \mathrm{~cm} / \mathrm{sec}$ near the deep boundary to approximately $25 \mathrm{~cm} / \mathrm{sec}$ in the vicinity of the shallow coast. As a brief study shows (see Appendix 2), the sheared flow affects dispersion characteristics and the cross-channel structure of the BSW in a rather complicated manner. For purposes of a simple-as-possible qualitative analysis, it is assumed that we can still use a uniform (across the channel) background flow and the corresponding $y$-structure of the BSW. However, the choice of the background flow amplitude, $U_{o}$, becomes somewhat arbitrary. We select $U_{o}$ which is of the order of the phase velocity of the first cross-channel BSW mode of the most energetic alongshore wavelengths ( 37.5 and $50 \mathrm{~km}$ ), and observe that such a choice is comparable to the amplitude of the background current in the vicinity of the maximum amplitude of the 
main forcing, $U \partial_{x}\left(\frac{f}{H}\right)$, in the vorticity equation (3.7). Across the channel, that maximum occurs near $y$ corresponding to the midshelf section. Based on this argument, the value of $U_{o}$ in all other experiments is taken to be equal to the amplitude of the background current at the midshelf section. In the basic experiment $U_{o} / c_{R}$ is of the order of 1 for the first cross-channel mode of the BSW's of 50,37.5, 30 and $25 \mathrm{~km}$ along-channel scale. The shorter waves are advected back and forth rather quickly $\left(U_{o} / c_{R}\right.$ is large) and can not gain appreciable amplitude. At long scales $(150$ and $75 \mathrm{~km}$ ) the energy is gained through the quasi-static geostrophic-like response and the generation of the cross-channel modes higher than the first, so that $U_{o} / c_{R} \sim 1$.

In summary, the most energetic alongshore scale of $v$ in an ideal case is found on the BSW dispersion diagram in a point of the 4 lines crossing: 1) a vertical line corresponding to one of the along-channel wavenumbers, $k_{T}$, present in the $x$-structure of the bumps; 2) a curve representing first BSW cross-channel mode as that which most closely corresponds to the cross-channel structure of the bumps; 3) a horizontal line depicting one of the resonant harmonics determined by the wind period; 4) an inclined straight line, $U_{o} k$, showing both an amplitude of the advective velocity, $U_{o}$, and the phase speed of the BSW and, thus, representing the ratio $U_{o} / c_{R}$, which should be approximately 1 . 


\section{Chapter 4}

\section{Dependence of the Offshore Flow Characteristics on the Governing Parameters.}

The magnitude and alongshore correlation scale of the time-variable offshore current $\left(v^{t}\right)$ as well as the ratio of amplitudes of the two components (cross- and along-channel) of the time dependent flow in the middle of the shelf are of the primary concern in this study. In this chapter, their sensitivity to the governing parameters of the model, namely, the amplitude and period of the wind, the amplitude and alongshore structure of the topographic irregularities, and friction, is discussed. In addition, other parameter combinations are then considered.

We estimate the amplitude of the time-variable cross-channel flow by its standard deviation, $\sigma\left(v^{t}\right)$, calculated for each point for a period of forcing and then averaged along the channel. Accordingly, the ratio of amplitudes of the two components of the time-dependent flow $\left(u^{t}\right.$ and $\left.v^{t}\right)$ is evaluated by the ratio of their standard deviations, $\sigma\left(v^{t}\right) / \sigma\left(u^{t}\right)$. Note that the amplitude of the alongshore velocity corresponds essentially to a standard deviation of the background flow. Finally, the alongshore correlation scale of $v^{t}$ is measured by the $e$-folding scale of its mean (over a period) along-channel autocorrelation function.

\subsection{Dependence on the wind amplitude. (Experiments 24,25).}

A variation of the wind amplitude causes a change in the amplitude of the background current, $U_{o}$. As equation (3.4) shows, $U_{o} \propto \tau_{1}$.

The response of the cross-channel flow to modification in $U_{o}$ is twofold. 
First, not surprisingly, an enhanced ambient flow produces proportionally larger crosschannel flow. Mathematically, this follows from (3.16) where non-dimensional $|v|$ should be multiplied by $U_{o}$ in order to receive a dimensional value. Hence, the amplitude of $v$ depends linearly on $U_{o}$. Indeed, the results of experiments show that the time-dependent cross-channel flow increases with the background current (Fig. 23a). The rate of increase varies across the channel but, in agreement with an estimate based on quasi-geostrophic theory, the rate of change of the time dependent cross-channel flow with $U_{o}$ is approximately constant (linear dependence) in the middle of the shelf $(y=10-30 \mathrm{~km})$, where $v^{t}$ reaches maximum magnitude.

Second, by affecting the ratio $U_{o} / c_{R}$, a variation in the amplitude of the background current influences the distribution of energy of $v^{t}$ between the alongshore scales and, thus, the alongshore correlation scale. For instance, if the two limits (short and long wavelength) are reached for some fixed values of $U_{o} / c_{R}$, then the ranges of the alongshore wavenumbers for which these limits hold are dependent on $U_{o}$ and shift toward smaller(larger) $k$ as $U_{o}$ increases(decreases), since $\frac{U_{o}}{c_{R}} \propto U_{o} k^{2}$. Similarly, the alongchannel wavelength containing most of the cross-channel flow energy increases with $U_{o}$ since $\frac{U_{o}}{c_{R}}=1$ for larger $c_{R}$, i.e., for smaller $k$. Therefore, it is expected that the alongshore correlation scale of the cross-channel flow increases as the background flow becomes stronger. The rate of the alongshore scale increase, again, can be estimated from the fact that for some particular value of $U_{o} / c_{R}$,

$$
k \propto \sqrt{1 / U_{o}}
$$

The results of the experiments 24,25 (Fig. 23c) are consistent with the statement about the increase of the $v^{t}$ along-channel correlation scale and, to some extent, support the estimated rate of that increase.

Finally, Figure 23b demonstrates that in the middle of the shelf $(y=10-30 \mathrm{~km})$ the ratio of amplitudes of the time-variable cross- and along-channel components is of the order of $10-20 \%$ for $\tau_{o}=0.5 \cdot 10^{-4} \mathrm{~m}^{2} / \mathrm{sec}^{2}$ and diminishes with increasing $\tau_{o}$. As we have seen, a stronger wind forces both along- and cross-channel flow of larger amplitude. Apparently (since $\sigma\left(v^{t}\right) / \sigma\left(u^{t}\right)$ diminishes with $\tau_{o}$ ), the rate of increase is larger for the time-dependent alongshore component. 


\subsection{Dependence on the wind period. (Experiments 1-5).}

A variation in the wind period is associated with a change of both the frequency and the amplitude of the background current.

The effect of $U_{o}$ has been considered above. In agreement with that discussion, the amplitude of $v^{t}$ increases with the period of wind (Fig. 24a) since $U_{o}$ also becomes stronger. The increase is not linear though, due to non-linear dependence of the background flow amplitude on the wind frequency in equation (3.4).

Again, in the middle of the shelf $(y=10-30 \mathrm{~km})$, the amplitude of $v^{t}$ diminishes relative to $u^{t}$ as $U_{o}$ becomes larger, i.e., as the wind period increases (Fig. 24b).

The alongshore correlation scale of $v^{t}$ increases (Fig. 24c) as the wind period varies from 5 to 20 days in accordance with the growth of $U_{o}$. However, that scale diminishes as the wind period increases from 2 to 5 days while $U_{o}$ still becomes stronger.

Such a behaviour of the alongshore correlation scale is related to the fact that, besides the effect of increase in amplitude of the ambient current, a change of the wind period restructures the set of the resonant harmonics. A dispersion diagram (Fig. 22) shows that as the frequency of forcing increases, the range of alongshore wavenumbers, for which a resonance is possible, narrows. Clearly, a decrease of the fundamental period from 20 to 5 days is associated with a cut off of the BSW's of short alongshore lengths, which are not important in the response anyway due to large values of $U_{o} / c_{R}$ for them. Hence, in this period range, the tendency in the alongshore correlation scale is determined by the change of $U_{o}$ and, in particular, by the decrease of the most energetic (in terms of $v$ ) scale, described by $k \propto \sqrt{1 / U_{o}}$. However, further decrease of the wind period (from 5 to 2 days) cuts off waves of all alongshore scales shorter than $50 \mathrm{~km}$ and, among them, those with $\frac{U_{o}}{c_{R}} \sim 1$ (most energetic otherwise). Essentially, the response becomes made of just longer waves and the correlation scale increases.

Thus, as long as the along-channel scales for which $\frac{U_{o}}{c_{R}} \sim 1$ remain within a range of wavenumbers available for response, despite the decrease of the wind period (for instance, from 20 to 5 days), the alongshore correlation scale diminishes (since $U_{o}$ diminishes and $\frac{U_{o}}{c_{R}} \sim 1$ for larger $k$ ). Otherwise, a decrease of the wind period (for instance, from 5 to 2 days) causes an increase in alongshore correlation scale of the cross-channel flow.

One more observation. On the dispersion diagram we consider a forcing frequency, $\omega_{F}$, as the lowest possible for the response, and the highest frequency, $\omega_{A}$, corresponding to 
that of intersection of the $U_{o} k$ with the vertical $k=k_{T}$ and the first cross-channel mode curve, as a Doppler shift of the possibly excited BSW. These two frequencies characterize the rate of local change and the strength of the alongshore advection, respectively. As long as $\omega_{F}$ is of the order of $\omega_{A}$ or less, lee waves can be efficiently generated and dominate the response. However, when $\omega_{F}$ is much larger than $\omega_{A}$ (as in experiment with 2 days period, for instance) lee waves can not be formed. The dynamics is completely linear, i.e., even the background current - perturbation flow interaction is unimportant. In that sense, this, say, high-frequency limit is similar to the long wavelength limit.

Finally, we note that the similar dependence of the cross-channel flow characteristics on the wind period is observed also in experiments with other, larger, values of $h_{o}$, the amplitude of the topographic irregularities (experiments 6-15),suggesting that dispersion properties of the BSWs, in particular, the narrowing of the alongshore wavenumber range with decreasing period and growth of $c_{R}$ with decrease of $k$, are qualitatively the same for the inspected values of $h_{o}$.

\subsection{Dependence on friction. (Experiments 16-20).}

The dependence of the characteristics of $v^{t}$ on the friction is tested for different periods by making simulations with a decreased value of the friction coefficient relative to the basic case. We have chosen a new value of $r=1.5 \cdot 10^{-4} \mathrm{~m} / \mathrm{sec}$ which is a half of the basic one.

The results for all five periods qualitatively are the same, so that we discuss them only for the basic period, $T=10$ days.

In essence, the dependence is determined by the variation of $U_{o}$. As the friction diminishes, $U_{o}$ becomes larger. Then, in agreement with an increase of $U_{o}$, the amplitude and the alongshore correlation scale of $v^{t}$ grow (Fig. $25 \mathrm{a}, \mathrm{c}$ ).

The ratio $\sigma\left(v^{t}\right) / \sigma\left(u^{t}\right)$ is about $10-20 \%$ in the middle of the shelf and does not change considerably between the two values of $r$ (Fig. 25 b). 


\subsection{Dependence on amplitude of the bumps. (Experiments 1-15).}

In order to study the dependence of the cross-channel flow characteristics on the bump amplitude $\left(h_{o}\right)$, in addition to simulations 1-5 in which the basic value of $h_{o}=11.4 \mathrm{~m}$ has been used, experiments 6-10 have been run with $h_{o}=30 m$ and experiments 11-15 with $h_{o}=45 \mathrm{~m}$.

Again, the dependence is qualitatively the same at all tested wind periods. Therefore, attention is limited to experiments with the basic wind period, $T=10$ days.

Hart's quasi-geostrophic theory implies that the amplitude of the cross-channel flow linearly depends on the amplitude of the bumps (see equation (3.16)). Our results show (Fig. 26 a) that, indeed, the magnitude of $v^{t}$ increases with $h_{o}$, although the functional dependence is not linear, presumably, due to the non-quasi-geostrophic effects.

Since the background flow, which dominates the variation of the along-channel current, is the same in these experiments, while the amplitude of the cross-channel flow grows with $h_{o}$, the ratio $\sigma\left(v^{t}\right) / \sigma\left(u^{t}\right)$ increases with $h_{o}$ and becomes about 0.3-0.6 in the middle of the shelf (Fig. $26 \mathrm{~b}$ ). Moreover, the ratio reaches values larger than 1 for the wind periods of 2 and 5 days.

The alongshore correlation scale of $v^{t}$ somewhat decreases with $h_{o}$ (Fig. $26 \mathrm{c}$ ), demonstrating that relatively more energy is contained at shorter alongshore scales. Probably, an increase of $h_{o}$ affects the ratio $U_{o} / c_{R}$ for a given wavenumber $k$. First, as $h_{o}$ becomes larger, the time mean background flow intensifies (Haidvogel and Brink, 1986). Accordingly, the amplitude of the time dependent flow, $U_{o}$, diminishes and $U_{o} / c_{R}$ becomes approximately 1 for a smaller $k$. Second, possibly, the topographic bumps of large amplitude may affect the dispersive properties of the BSWs so that for a given $k$ the waves become faster as $h_{o}$ increases causing the necessary change in $U_{o} / c_{R}$. (We did not elaborate on this. For instance, Brink, 1980 studied the influences of topographic irregularities on dispersive properties of the BSW, addressing the problem of the BSW scattering by the bottom bumps. His calculations show that the BSW dispersion characteristics and, in particular, their phase speed change linearly with $h_{o}$.) 


\subsection{Sensitivity to the spectral shape of the bumps alongshore structure. (Experiment 26).}

In experiment 26 a different spectral shape of the alongshore structure of the topographic irregularities ( $k_{T}^{-3}$ instead of the $k_{T}^{-2}$ in the basic case) is used. The total variance of the bumps is preserved, so that the amplitude of the topographic disturbances of the first alongshore mode (150 $\mathrm{km}$ wavelength) is amplified while at all other scales the amplitude is decreased.

Accordingly, the alongshore correlation scale of $v^{t}$ increases (Fig. 27 c), i.e., larger portion of the fluctuating cross-channel flow energy is contained in the long $x$-scales.

Meanwhile, the amplitude of the time dependent offshore current decreases (Fig. 27 a)indicating that $v^{t}$ is produced less effectively on the larger alongshore scales, presumably, due to less effective generation of lee waves.

\subsection{Sensitivity to a non-monochromatic wind spectrum. (Ex- periments 27,28$)$.}

\section{Two frequency wind.}

In experiment 27 the idealization of monochromatic wind spectra is relaxed and the wind time dependence is represented by two frequency components of different amplitudes: $75 \%$ of the wind energy is supplied by the 14 days period and $25 \%$ by the 4 days period. The total wind stress variance is the same as in the experiment 4 with monochromatic (14 days period) wind. (Results of experiment 4 are used for comparison here.)

The studied characteristics of the cross-channel flow remain almost unchanged relative to the monochromatic wind. Note although, that standard deviation of $v^{t}$ somewhat decreases and correlation scale slightly increases in the two-frequency case (Fig. 28 a,c). Why does this happen?

Apparently, the amplitude of $U_{o}$, corresponding to 14 days period, decreases from about $25 \mathrm{~cm} / \mathrm{sec}$ to about $20 \mathrm{~cm} / \mathrm{sec}$ as a result of providing less wind energy at this frequency. The decrease in $U_{o}$ is associated with diminishing of the amplitude of the resulting cross-channel flow and of the alongshore correlation scale. Meantime, by supplying a portion of the energy at a shorter (4 days) period, we completely "cut off" the 
alongshore wavelengths smaller than about $20 \mathrm{~km}$ from those available for response. As a result, the correlation scale should increase. In addition, $U_{o}$ produced from the input of $25 \%$ of the wind energy through the 4 days period is rather small (about $3.5 \mathrm{~cm} / \mathrm{sec}$ ), so that lee waves are generated inefficiently and the $v^{t}$ amplitude decreases.

A combination of these two effects, as results show, amounts to the decrease in the cross-channel flow amplitude and increase of its correlation scale.

\section{Real wind.}

In experiment 28 the low-frequency wind stress data (with time-mean subtracted) for 120 days of observations from the CODE-2 are used to force the circulation in the channel with the geometry the same as in the basic experiment. The main purpose of this experiment is to see the quantitative characteristics of the cross-channel flow resulted from application of the real wind and, in particular, with a continuous spectrum of frequencies. The last 40 days of the wind stress time series, along with the $x$-averaged along-channel flow component, which can be used as the background current estimate, are depicted in Figure 29. Note, that relative to the wind stress, the amplitude of approximately 2 days period oscillations of $\bar{U}^{x}$ is smaller, so that a larger portion of the $\bar{U}^{x}$ energy is contained in longer periods (roughly, $5-20$ days). It follows from the previous analysis, that for such periods the amplitude of the background flow plays a decisive role in determining the magnitude and the correlation scale of the cross-channel flow. In this experiment, $\bar{U}^{x}$ is about $35 \mathrm{~cm} / \mathrm{sec}$ and of the other simulations is closest to experiment 25 with wind period 10 days and the amplitude $2 \cdot 10^{-4} \mathrm{~m}^{2} / \mathrm{sec}^{2}$. Presumably, due to this reason all the characteristics of $v^{t}$ are quite similar in these two experiments (compare Figure 30 with Figure 23).

\subsection{Addition of non-zero time mean wind. (Experiments 29,30).}

Experiments 29,30 were carried out with the wind containing non-zero time mean and oscillations with a 10 day period. The total wind stress variance, again, was the same as in the basic experiment. The time mean wind supplied $3 / 4$ of the total energy while the oscillations accounted for $1 / 4$ of the total energy. Calculations were made for 140 days and the results are provided for the last 20 days when the circulation in the middle of the shelf did not change noticably between consecutive two periods of forcing.

A negative time mean component of wind (experiment 29) favors the excitation of 
lee waves. Consequently, a vigorous time mean cross-channel circulation develops which can be seen in the $t-x$ diagram of the total offshore velocity (Fig. 31a). Averaged in time and along the channel, the alongshore flow is about $21 \mathrm{~cm} / \mathrm{sec}$ on the mid-shelf section. Then, according to the BSW dispersion diagram (Fig. 22), lee waves of the first cross-channel mode have alongshore scales 37.5 and $50 \mathrm{~km}$. Figure 31a suggests that the alongshore scale of the total cross-channel flow is comparable to that estimate.

The $t-x$ diagram of the fluctuating component of $v$ (Fig. 31b) reveals quite a regular pattern: the positive and negative "cells" 1) are of approximately the same amplitude, 2) alternate in time following oscillations in the background flow and 3) have alongchannel scale coincident with that of the total cross-channel flow. In fact, the mean autocorrelation function of $v^{t}$ (Fig. 32) has a well determined maximum at $40.6 \mathrm{~km}$ which can be regarded as the alongshore scale of the time dependent cross-channel flow and is consistent with the estimate of the $x$-scale of the excited lee waves.

A careful comparison of the two $t-x$ diagrams, for the total cross-channel flow and for its time dependent component (Fig. 31a,b), shows that anomalies of $v$ are situated at the along-channel locations where the offshore flow changes sign. In essence, these locations correspond to the centers (or axes) of the positive or negative topographic vortices (or meanders in vorticity field). In the background flow which opposes to propagation of the BSW's, the vortices are shifted southward relative to topography as has been described by Hart (1990) and mentioned in the discussion of the long scale response (section 3.4.2). For a fixed value of friction, the shift is larger (smaller) for a stronger (weaker) southward background current, so that the position of the vorticies undulates and gives rise to the oscillations in cross-channel flow.

If the mean wind is positive (Experiment 30), lee waves do not form. As a result, the cross-shelf currents are much weaker (Figure 33a) than that caused by the negative time mean wind. The magnitude of the fluctuating component of $v$ is essentially negligible (Figure 33b). In essence, the results of this experiment, once again demonstrate the importance of lee waves in the generation of cross-channel flow of considerable amplitude. 


\section{Chapter 5}

\section{Conclusions.}

The goal of this study was to explore the physics and to obtain quantitative characteristics of topographic influencies on time dependent wind-driven offshore flow.

First, we analyzed the results of the basic experiment to clarify what kind of physics determines the response in the system as a whole and, then, at the different alongshore scales. It has been shown that the long scale (geostrophic) response contributes mostly to the time dependent component of $v$ and is relevant for the 150 and $75 \mathrm{~km}$ alongshore scales in the basic experiment. In contrast, the adjustment at short scales is due to changes in the relative vorticity of water columns advected over a rough bottom and gives rise mainly to the time mean cross-channel flow. In the model, the short scale response takes place at alongshore lengths smaller than, approximately, $20 \mathrm{~km}$. In most cases, the portion of the offshore flow generated at the short scales is negligible. As a rule, the most energetic cross-channel currents are produced at intermediate scales $(25-50 \mathrm{~km}$ in the model) due to efficient excitation of lee waves.

An analysis of the vorticity equation revealed some similarity of the problem at hand to a quasi-geostrophic model studied by Hart (1990). Hart's solution has been adopted and allowed fruitful diagnostic application to the BSW dispersion diagram to determine the separation of different scales and to interpret the results of the model experiments. Summarizing, the topographic irregularities establish a "potential" spatial structure of the response (first cross-channel mode BSW's with the alongshore wavenumbers corresponding to $k_{T}$ ) which is finally chosen by the wind-driven background current. The wind stress determines the period and amplitude of $U$, which, in turn, sets the resonant harmonics and the most energetic alongshore scale of $v^{t}$ that usually, but not necessarily 
always, corresponds to the wavelength at which $U_{o} / c_{R}=1$. In essence, the separation of scales into long, short and intermediate is based on comparison of the magnitude of the geostrophic term (advection of the background vorticity by perturbation flow) with the change in relative vorticity which is established by the ratio $U_{o} / c_{R}$ :

$\frac{U_{o}}{c_{R}} \ll 1$ determines the long scale (geostrophic) limit at which the amplitude of $v$ increases with $k$. Almost all energy of $v$ at these scales is in the fluctuating component of the cross-shelf velocity and this contribution is rather substantial.

$\frac{U_{o}}{c_{R}} \gg 1$ corresponds to the short scale (relative vorticity) limit with amplitude of $v$ diminishing with $k$. These scales contribute mainly to the time mean component of $v$. Our calculations show that the amplitude of $v$ at these scales is negligible.

$\frac{U_{o}}{c_{R}} \sim 1$ characterizes the intermediate scales where lee wave dynamics is most pronounced. The most energetic alongshore scale of $v$ is found in the vicinity of $U_{o} / c_{R}=1$ and has the largest contribution both to the time mean and fluctuating component of the cross-channel flow.

Analysis of the basic experiment shows that lee waves were effectively excited at the length scale ranging from approximately 25 to $50 \mathrm{~km}$. Then, with $U_{o} \approx 20 \mathrm{~cm} / \mathrm{sec}$, the intermediate scales are determined by, roughly, $0.3 \leq \frac{U_{o}}{c_{R}} \leq 3$.

In general, the magnitude of the time variable offshore current increases with the wind amplitude and period, with decreasing friction (in essence, due to growth in the background flow amplitude in all three cases) and with increasing amplitude of the topographic irregularities. The largest value of $v^{t}$, about $5.5 \mathrm{~cm} / \mathrm{sec}$, among all simulations, was reached in experiment 15 with $h_{o}=45 \mathrm{~m}$ (more than $50 \%$ of the mean depth) and the wind period 20 days. (For comparison, Martell and Allen (1979), using digitized topographic data for the Oregon shelf from Peffley and O'Brien (1976), calculated an amplitude of approximately $40 \mathrm{~m}$ for the alongshore wavelength about $30 \mathrm{~km}$ and mean depth $100 \mathrm{~m}$.) In this experiment and in an experiment with $h_{o}=30 \mathrm{~m}, v^{t}$ constituted $40-60 \%$ of the alongshore current amplitude in the middle of the shelf, indicating the possibility of generation of considerable temporally varying offshore flow due to irregular bottom topography. In most of the experiments, however, the ratio $\sigma\left(v^{t}\right) / \sigma\left(u^{t}\right)$ was $10-20 \%$ with exception of the higher values, $20-30 \%$, for the shortest period of forcing, $T=2$ days, when the alongshore current is rather weak. 
The along-channel correlation scale of $v^{t}$, determined as an $e$-folding distance of its autocorrelation function, varied between about $5.5 \mathrm{~km}$ (experiment 24, small wind amplitude) and $11 \mathrm{~km}$ (experiment 26, spectrum of the bumps varies as $k_{T}^{-3}$ ). Again, for comparison, Dever (1995), using velocity observations over the Northern California shelf, estimates the correlation scale of the time variable cross-shelf current between 4 and $30 \mathrm{~km}$. In addition, he finds some support for a statement that the larger of those scales, $15-30 \mathrm{~km}$, is determined by the corresponding spatial wind variations. He left smaller correlation scales unexplained.

Experiment 29, with non-zero negative mean wind included, as well as experiments with simple structure of the topographic irregularities, demonstrate that in certain circumstances, the $v^{t}$ autocorrelation function can exhibit a well-determined maximum close to the most energetic alongshore scale of $v^{t}$. However, if the energy of the cross-channel flow spreads out between several alongshore lengths, the secondary maximum of the autocorrelation function becomes insignificant or disappears.

Dependence of the characteristics of the time variable offshore current on several governing parameters has been considered.

We emphasize that our results, obtained for a continuum of bottom bumps, supplement, rather than oppose, results that consider the influences of isolated topographic features on the flow (e.g., among coastal studies: Martell and Allen, 1979; Wilkin and Chapman, 1990; in general circulation: Thompson, 1990). For example, our results are hardly applicable to the Coastal Ocean Dynamics Experiment (CODE), the site for which (Fig. 34) was chosen, among other reasons, due to relatively straight (in the alongshore direction) bottom topography. Meanwhile, the abrupt changes in the coast line orientation and in the bottom topography evident both to the South and to the North of the CODE area can be interpreted as isolated topographic features. The length scale of the influence of such a feature depends, in particular, on the size of the feature itself and on the frictional decay scale. In a quasi-geostrophic case, assuming that the dynamics is dominated by a resonant lee wave, the wavelength corresponds to $L^{2}=\frac{U_{o} D}{f H_{y}^{b}}$. The frictional scale, $L^{f}$, comes from assumption that $\hat{r}=1$ in (3.12). Then $L^{f}=\frac{U_{o}}{f} \frac{D}{\delta_{E}}$, or $L^{f}=\frac{H_{y}^{b}}{\delta_{E}} L^{2}$. For the representative values of $\delta_{E} \sim 10 \mathrm{~m}, L^{f}$ ranges from $10 \mathrm{~km}$ $\left(H_{y}^{b} \sim 10^{-3}, L \sim 10 \mathrm{~km}\right)$ to about $300 \mathrm{~km}\left(H_{y}^{b} \sim 10^{-2}, L \sim 100 \mathrm{~km}\right)$ showing that 
disturbances excited by an isolated topographic feature can play a noticeable role in the dynamics of quite distant regions.

In addition, it is not well understood how the results might change in the presence of stratification. For instance, Samelson and Allen (1987) note that a typical $N$, the Brunt-Väisälä frequency, over the shelf ranges from $10^{-3}$ to $5 \cdot 10^{-2} \mathrm{sec}^{-1}$. Then the vertical decay scale of linear bottom-trapped disturbances estimated as $\frac{f L}{N}$ even for the shortest wavelength considered here $(10 \mathrm{~km})$ and $N=5 \cdot 10^{-2} \mathrm{sec}^{-1}$ is $200 \mathrm{~m}$. Therefore, in this sense, the barotropic analysis may not be a bad approximation especially for depths shallower than $200 \mathrm{~m}$ and/or weaker stratification. On the other hand, however, the dispersion properties of the Coastally Trapped waves differ from those of BSW's quantitatively and, for strong stratification, even qualitatively (e.g., Chapman, 1983) so that, for instance, the generation of lee waves may not be possible at all. Also, in the presence of stratification, new possibilities arise for instabilities to develop. Then the results of the barotropic analysis presented here can be strongly affected by stratification. 


\section{Appendix 1. Governing parameters in numerical experiments.}

\begin{tabular}{|c|c|c|c|c|c|c|}
\hline & $\begin{array}{l}\text { Wind } \\
\text { period, } \\
\text { days }\end{array}$ & $\begin{array}{l}\text { Wind stress } \\
\text { amplitude, } \\
10^{-4} \mathrm{~m}^{2} \mathrm{sec}^{-2}\end{array}$ & $\begin{array}{c}\text { Bumps } \\
\text { amplitude, } \\
h_{o}, m \\
\end{array}$ & $\begin{array}{c}\text { Alongshore } \\
\text { wavenumbers } \\
\text { in bumps }\end{array}$ & $\begin{array}{c}\text { Alongshore } \\
\text { spectrum } \\
\text { of bumps }\end{array}$ & $\begin{array}{c}\text { Friction } \\
\text { coeff. } \\
r, m \cdot \sec ^{-1}\end{array}$ \\
\hline 1 & 2 & 1 & 11.4 & $1-15$ & $k^{-2}$ & $3 \cdot 10^{-4}$ \\
\hline 2 & 5 & 1 & 11.4 & $1-15$ & $k^{-2}$ & $3 \cdot 10^{-4}$ \\
\hline 3 & 10 & 1 & 11.4 & $1-15$ & $k^{-2}$ & $3 \cdot 10^{-4}$ \\
\hline 4 & 14 & 1 & 11.4 & $1-15$ & $k^{-2}$ & $3 \cdot 10^{-4}$ \\
\hline 5 & 20 & 1 & 11.4 & $1-15$ & $k^{-2}$ & $3 \cdot 10^{-4}$ \\
\hline 6 & 2 & 1 & 30 & $1-15$ & $k^{-2}$ & $3 \cdot 10^{-4}$ \\
\hline 7 & 5 & 1 & 30 & $1-15$ & $k^{-2}$ & $3 \cdot 10^{-4}$ \\
\hline 8 & 10 & 1 & 30 & $1-15$ & $k^{-2}$ & $3 \cdot 10^{-4}$ \\
\hline 9 & 14 & 1 & 30 & $1-15$ & $k^{-2}$ & $3 \cdot 10^{-4}$ \\
\hline 10 & 20 & 1 & 30 & $1-15$ & $k^{-2}$ & $3 \cdot 10^{-4}$ \\
\hline 11 & 2 & 1 & 45 & $1-15$ & $k^{-2}$ & $3 \cdot 10^{-4}$ \\
\hline 12 & 5 & 1 & 45 & $1-15$ & $k^{-2}$ & $3 \cdot 10^{-4}$ \\
\hline 13 & 10 & 1 & 45 & $1-15$ & $k^{-2}$ & $3 \cdot 10^{-4}$ \\
\hline 14 & 14 & 1 & 45 & $1-15$ & $k^{-2}$ & $3 \cdot 10^{-4}$ \\
\hline 15 & 20 & 1 & 45 & $1-15$ & $k^{-2}$ & $3 \cdot 10^{-4}$ \\
\hline 16 & 2 & 1 & 11.4 & $1-15$ & $k^{-2}$ & $1.5 \cdot 10^{-4}$ \\
\hline 17 & 5 & 1 & 11.4 & $1-15$ & $k^{-2}$ & $1.5 \cdot 10^{-4}$ \\
\hline 18 & 10 & 1 & 11.4 & $1-15$ & $k^{-2}$ & $1.5 \cdot 10^{-4}$ \\
\hline 19 & 14 & 1 & 11.4 & $1-15$ & $k^{-2}$ & $1.5 \cdot 10^{-4}$ \\
\hline 20 & 20 & 1 & 11.4 & $1-15$ & $k^{-2}$ & $1.5 \cdot 10^{-4}$ \\
\hline 21 & 10 & 1 & 11.4 & 1,2 & $k^{-2}$ & $3 \cdot 10^{-4}$ \\
\hline 22 & 10 & 1 & 11.4 & $4-6$ & $k^{-2}$ & $3 \cdot 10^{-4}$ \\
\hline 23 & 10 & 1 & 30 & 15 & & $3 \cdot 10^{-4}$ \\
\hline 24 & 10 & 0.5 & 11.4 & $1-15$ & $k^{-2}$ & $3 \cdot 10^{-4}$ \\
\hline 25 & 10 & 2 & 11.4 & $1-15$ & $k^{-2}$ & $3 \cdot 10^{-4}$ \\
\hline 26 & 10 & 1 & 11.4 & $1-15$ & $k^{-3}$ & $3 \cdot 10^{-4}$ \\
\hline 27 & 4,14 & $1 / 4,3 / 4$ of total energy & 11.4 & $1-15$ & $k^{-2}$ & $3 \cdot 10^{-4}$ \\
\hline 28 & CODE-2 & $\mathrm{rms} 1.25$ & 11.4 & $1-15$ & $k^{-2}$ & $3 \cdot 10^{-4}$ \\
\hline 29 & negative mean, 10 & $3 / 4,1 / 4$ of total energy & 11.4 & $1-15$ & $k^{-2}$ & $3 \cdot 10^{-4}$ \\
\hline 30 & positive mean, 10 & $3 / 4,1 / 4$ of total energy & 11.4 & $1-15$ & $k^{-2}$ & $3 \cdot 10^{-4}$ \\
\hline
\end{tabular}




\section{Appendix 2. Influence of a steady sheared background flow on the BSW dispersion characteristics.}

Consider a problem of BSW interaction with a steady nonuniform (across the channel) background current over an exponential bottom topography. The vorticity equation for the free, inviscid BSW can be written as:

$$
\left[\partial_{t}+U(y) \partial_{x}\right]\left[\frac{\zeta^{\prime}}{H^{b}(y)}\right]+v\left[\frac{f+\bar{\zeta}}{H^{b}(y)}\right]_{y}=0,
$$

where $\zeta$ and $\zeta^{\prime}$ are the background and perturbation relative vorticity respectively (as in equation (3.7)). Introduce a streamfunction, $\psi_{y}=-u H^{b}, \psi_{x}=v H^{b}$, and look for a harmonic solution: $\psi(x, y, t)=\phi(y) e^{i k(x-c t)}$. Then, the vorticity equation (A1) becomes:

$$
[U(y)-c]\left[\phi_{y y}-\phi_{y} \frac{H_{y}^{b}}{H^{b}}-k^{2} \phi\right]+\phi\left[-U_{y y}-\left(f-U_{y}\right) \frac{H_{y}^{b}}{H^{b}}\right]=0,
$$

which may be written as

$$
[U(y)-c] q^{\prime}+\phi \bar{q}_{y}=0 .
$$

where $\bar{q}$ and $q^{\prime}$ are the background and perturbation total vorticity respectively, and solved numerically.

This is a standard normal mode barotropic instability problem. For some typical shelf conditions it has been solved analytically by Tareev (1971). We calculated the phase speed and the structure of the normal modes for several $y$-profiles of the background current $U(y)$ determined by equation (3.4) corresponding to some particular time. First of all, it turned out that the necessary condition for instability to occur, $\bar{q}_{y}=0$ within a channel, is not satisfied, so that the waves generated by the present sheared background flow are stable.

Two examples of the BSW dispersion diagram for the first 5 cross-channel modes are depicted in Figure A.1 for $U(y)$ at $t=3.9$ days and $t=8.9$ days when an averaged (across the channel) ambient current reaches its maximum (no-lee-wave phase) and minimum (lee-wave phase) respectively. For comparison, the dispersion diagrams of BSW's in a uniform ambient flow (y-averaged value of $U(y)$ is used) are shown. Clearly, the frequency (and so phase speed) of the BSW's increases in a sheared background flow no matter what 
sense of that shear is, positive or negative. The shear itself does not significantly affect a magnitude of the ambient vorticity gradient: terms $U_{y y}$ and $U_{y} \frac{H_{y}^{b}}{H^{b}}$ are at least an order of magnitude less than $f \frac{H_{y}^{b}}{H^{b}}$. Rather, a structure of the normal modes changes depending on the shape of the shear and allows them to select a virtual advective velocity which is different from that averaged across the channel. That choice becomes an intrinsic property of the system once a background current profile is provided.

However, in a time variable background flow like that determined by (3.4) the shear constantly evolves throughout a period and so does the structure of the normal modes. Then, it is unclear whether is it possible to apply an analysis similar to that in Section 3.4.3 which is based on assumption of the uniform background current and uses the property that the cross-channel structure of the BSW does not depend on $U$. 


\section{References}

Battistiti, D.S., and B.M. Hickey, 1984. Application of remote wind-forced coastal trapped wave theory to the Oregon and Washington coasts. J. Phys. Oceanogr., 14, 887-903.

Brink, K.H., 1980. Propagation of barotropic continental shels waves over irregular bottom topography. J. Phys. Oceanogr., 10, 765-778.

Brink, K.H., J.H. LaCasce, and J.D. Irish, 1994. The effect of short-scale wind variations on shelf currents. J. Geophys. Res., 99, 3305-3315.

Chapman, D.C., 1983. On the influence of stratification and continental shelf and slope topography on the dispersion of subinertial coastally trapped waves. J. Geophys. Res., 13, 1641-1652.

Chapman, D.C., 1987. Application of wind-forced, long, coastal-trapped wave theory along the California coast. J. Geophys. Res., 92, 1798-1816.

Charney, J.G., and J.G. DeVore, 1979. Multiple flow equilibria in the atmosphere and blocking. J. Atmos. Sci., 36, 1205-1216.

Dever, E.P., 1995. Subtidal cross-shelf circulation on the Northern California shelf. Ph.D. dissertation, Joint Program in Oceanography/Applied Ocean Science and Engineering, Massachusetts Institute of Technology, Cambridge, Mass., Woods Hole Oceanographic Institution, Woods Hole, Mass., 157 pp.

Gill, A.E., and E.H. Schumann, 1974. The generation of long shelf waves by wind. J. Phys. Oceanogr., 4, 83-90.

Haidvogel, D.B., and K.H. Brink, 1986. Mean currents driven by topographic drag over the continental shelf and slope. J. Phys. Oceanogr., 16, 2159-2172.

Haidvogel, D.B., J.L. Wilkin, and R. Young, 1991. A Semi-spectral primitive equation ocean circulation model using vertical sigma and orthogonal curvilinear horizontal coordinates. J. Comput. Phys., 94, 151-185. 
Hart, J.E., 1979. Barotropic quasi-geostrophic flow over anisotropic mountains. J. Atmos. Sci., 36, 1736-1746.

Hart, J.E., 1990. On oscillatory flow over topography in a rotating fluid. J. Fluid Mech., 67, 437-455.

Hedström, K.S., 1994. User's manual for a Semi-spectral primitive equation ocean circulation model. Version 3.9. Tech. Rep. \#93-23, Institute of Marine and Coastal Sciences, Rutgers University, 131 pp.

Lentz, S.J., 1987. A description of the 1981 and 1982 spring transitions over the Northern California shelf. J. Geophys. Res., 92, 1545-1567.

Martell, C.M., and J.S. Allen, 1979. The generation of continental shelf waves by alongshore variations in bottom topography. J. Phys. Oceanogr., 9, 696-711.

Mitchum, G.T., and A.J. Clarke, 1986. Evaluation of frictional, wind forced long wave theory on the West Florida shelf. J. Phys. Oceanogr., 16, 1029-1037.

Pedlosky, J., 1981. Resonant topographic waves in barotropic and baroclinic flows. J. Atmos. Sci., 12, 2626-2641.

Pedlosky, J., 1987. Geophysical fluid dynamics. Springer-Verlag, 710pp.

Peffley, M.B., and J.J. O'Brien, 1976. A three-dimensional simulation of coastal upwelling off Oregon. J. Phys. Oceanogr., 6, 164-180.

Rambaldi, S., and G.R. Flierl, 1981. Form drag instability and multiple equilibria in the barotropic case. Il Nuovo Cimento, 6, 505-522.

Samelson, R.M., and J.S. Allen,1987. Quasi-geostrophic topographically generated mean flow over a continental margin. J. Phys. Oceanogr., 17, 2043-2064.

Tareev, B.A., 1971. Gradient-vorticity waves on the continental shelf. Izv. Akad. Nauk SSSR, Fiz. Atmos. Okeana, 7, 431-436 (in Russian).

Wilkin, J.L., and D.C. Chapman, 1990. Scattering of coastal-trapped waves by irregularities in coastline and topography. J. Phys. Oceanogr., 20, 396-421. 
Winant, C.D., 1983. Longshore coherence of currents on the Southern California shelf during the summer. J. Phys. Oceanogr., 13, 54-64. 


\section{List of Figures.}

Figure 1. A sketch depicting the coastal channel geometry. (From Haidvogel and Brink, 1986.)

Figure 2. (a) Bottom topography $(m)$ in the channel with parameters corresponding to the Basic experiment (3). Dashed lines depict sections in the mid-shelf and near the deep boundary where data have been collected; (b) ratio of the topographic disturbance to the background depth (\%).

Figure 3. Evolution of the streamfunction field through a period of forcing: (a) $t=$ 90 days; (b) $t=93$ days; (c) $t=95$ days; (d) $t=98$ days. Solid lines correspond to positive values; dashed lines - to negative values. (Basic experiment.)

Figure 4. Alongshore flow on the mid-shelf section for a period of forcing $(\mathrm{cm} / \mathrm{sec})$. Solid lines correspond to positive values; dashed lines - to negative values; dotted lines - to $0 \mathrm{~cm} / \mathrm{sec}$; dash-dot lines - to axes of the most pronounced topographic ridges or canyons. The same is true for other $t-x$ diagrams, unless otherwise stated. (Basic experiment.)

Figure 5. Cross-channel flow $(\mathrm{cm} / \mathrm{sec})$ in the deep part of the channel, depth about $2000 \mathrm{~m}$. (Basic experiment.)

Figure 6. (a) Cross-channel flow $(\mathrm{cm} / \mathrm{sec})$ in the middle of the shelf. (b) Fluctuating component of the cross-channel flow (total - time mean) at the same location. (Basic experiment.)

Figure 7. Flow components $(\mathrm{cm} / \mathrm{sec})$ on the mid-shelf section at $x=50 \mathrm{~km}$ : (a) crosschannel current; (b) along-channel current. Dashed line corresponds to the background current. (Basic experiment.)

Figure 8. (a) Time-mean alongshore currents $(\mathrm{cm} / \mathrm{sec})$; (b) standard deviation of alongshore currents $(\mathrm{cm} / \mathrm{sec})$. (Basic experiment.)

Figure 9. Difference between the total alongshore flow and the background current $(\mathrm{cm} / \mathrm{sec})$ in the middle of the shelf. (Basic experiment.) 
Figure 10. (a) Components of averaged (over a period and over the channel) $x$-momentum balance $\left(10^{-4} \mathrm{~m}^{2} / \mathrm{sec}^{2}\right)$. Terms of the averaged along the channel $x$-momentum balance $\left(10^{-3} m^{2} / \sec ^{2}\right)$ : (b) ${\overline{H \phi_{x}}}^{x}$, (c) $r \bar{u}^{x}$, (d) $\frac{\partial}{\partial y} \overline{(H u v)}^{x}$, (e) ${\overline{(H u)_{t}}}^{x}$. (Basic experiment.)

Figure 11. (a) Mean over a period offshore currents $(\mathrm{cm} / \mathrm{sec})$; (b) standard deviation of cross-channel currents $(\mathrm{cm} / \mathrm{sec})$. (Basic experiment.)

Figure 12. Sketchs of the relative vorticity response with (a) positive and (b) negative background flow; and of the geostrophic response with (c) positive and (d) negative background flow.

Figure 13. Mean (over the channel length) with a range given by \pm standard deviation terms in the vorticity balance (3.7) at the mid-shelf section for one period of forcing: (a) $\partial_{t}\left(\frac{\zeta^{\prime}}{H}\right),($ b $) \partial_{t}\left(\frac{\zeta}{H}\right),($ c $) U \partial_{x}\left(\frac{\zeta}{H}\right),(\mathrm{d}) U \partial_{x}\left(\frac{\zeta^{\prime}}{H}\right),($ e $) U \partial_{x}\left(\frac{f}{H}\right),(\mathrm{f})(\vec{u} \cdot \nabla) \frac{\varsigma}{H}$. (Basic experiment.)

Figure 14. The same as in Figure 13 but for (a) $(\vec{u} \cdot \nabla) \frac{\zeta^{\prime}}{H}$, (b) $(\vec{u} \cdot \nabla) \frac{f}{H}$, (c) $\frac{\tau^{x}}{H} \partial_{y}\left(\frac{1}{H}\right)$, (d) $\frac{r}{H} \partial_{y}\left(\frac{U}{H}\right),\left(\right.$ e) $\frac{r}{H}\left(\nabla \times \frac{\vec{u}}{H}\right),($ f)imbalance.

Figure 15. The same as in Figure 13 but for different physically meaningful combinations of terms: (a)the background current balance (3.10), (b)the balance associated with linear, non-viscous interaction of the background current with perturbation flow and topographic disturbances: $\left(\partial_{t}+U \partial_{x}\right)\left(\frac{\zeta^{\prime}}{H}\right)+(\vec{u} \cdot \nabla) \frac{f}{H}=-U \partial_{x}\left(\frac{f}{H}\right),(\mathrm{c})$ sum of non-linear term and friction due to perturbation: $(\vec{u} \cdot \nabla) \frac{\zeta^{\prime}}{H}+\frac{r}{H}\left(\nabla \times \frac{\vec{u}}{H}\right)$.

Figure 16. Time-mean amplitudes of alongshore Fourier components of terms in vorticity balance (3.11): (a) $\partial_{t}\left(\frac{\zeta^{\prime}}{H}\right),\left(\right.$ b) $U \partial_{x}\left(\frac{\zeta^{\prime}}{H}\right),($ c $) U \partial_{x}\left(\frac{f}{H}\right),(\mathrm{d})(\vec{u} \cdot \nabla) \frac{\zeta^{\prime}}{H},(\mathrm{e})(\vec{u} \cdot \nabla) \frac{f}{H}$, (f) $\frac{r}{H}\left(\nabla \times \frac{\vec{u}}{H}\right)$.

Figure 17. (a) The $t-x$ diagram of the cross-channel flow $(\mathrm{cm} / \mathrm{sec})$ from experiment 23 demonstrating short scale (relative vorticity) response; (b) a wavelength of topographic perturbation which is constructed from single sinusoid in this experiment. Both (a) and (b) taken from the mid-shelf section.

Figure 18. (a) the same as in Figure 17a but for experiment 21 (long wave response demonstration); (b) topographic perturbation due to a sum of mode 1 and 2; (c) 
amplitude of $v$ Fourier components averaged over $t=98-100$ days (lee wave phase) - solid line, $t=93$ - 95 days (no-lee wave phase) - dashed line and over whole period - dash-dot line.

Figure 19. The same as in Figure 18 but for experiment 22 in which bottom bumps represented by the intermediate scales disturbances: modes 4,5 and 6 (alongshore wavelengths $37.5,30$ and $25 \mathrm{~km}$, respectively).

Figure 20. For experiment 22 (intermediate scale response): (a) time mean offshore currents $(\mathrm{cm} / \mathrm{sec})$; (b) standard deviation of cross-channel currents $(\mathrm{cm} / \mathrm{sec})$.

Figure 21. Basic experiment, mid-shelf section:(a) evolution of $v$ alongshore spectrum for a period; (b) mean (over a period) alongshore spectrum of the total crosschannel flow (solid line) and the fluctuating component (dashed line); (c) portion of the fluctuating energy component of total $v$ (mean over a period alongshore number spectrum).

Figure 22. Barotropic Shelf Wave dispersion diagram for the background (exponential) topography.

Figure 23. Dependence of the cross-channel flow characteristics on the wind amplitude (experiments 3,24 and 25): (a) standard deviation of the fluctuating component of $v$ as a function of cross-channel distance; (b) its ratio to the amplitude of the alongshore fluctuating component vs. $y$; (c) alongshore correlation distance $(e-$ folding scale of the $v^{t}$ autocorrelation function) at the mid-shelf section.

Figure 24. The same as in Figure 23 but for experiments 1-5: dependence on the wind period.

Figure 25. The same as in Figure 23 but for experiments 16-20: dependence on friction.

Figure 26. The same as in Figure 23 but for experiments 3,8 and 13: dependence on the bump amplitude.

Figure 27. The same as in Figure 23 but for experiments 3 and 26: sensitivity to the bump spectral shape. On the $x$-axis 2 corresponds to topography with $k_{T}^{-2}$ spectrum, while 3 to $k_{T}^{-3}$ spectrum. 
Figure 28. The same as in Figure 23 but for experiments 4 (14 days wind period) and 27 (two-frequency wind: 4 and 14 days period). On the $x$-axis 1 corresponds to experiment $4 ; 2$ - to experiment 27.

Figure 29. (a) last 40 days of CODE-2 low-frequency wind stress data used in experiment 28; (b) $x$-averaged alongshore flow resulted from the forcing by the CODE-2 wind.

Figure 30. The same as in Figure 23 but for experiments 25 and 28 . On the $x$-axis 1 corresponds to experiment 25 (10 days wind period with $2 \cdot 10^{-4} \mathrm{~m}^{2} / \mathrm{sec}^{2}$ to experiment $4 ; 2$ - to experiment 28 (CODE-2 wind data).

Figure 31. The $t-x$ diagram for (a) the total cross-shelf flow; (b) its fluctuating component. Experiment 29: $75 \%$ of wind energy is supplied by the negative mean wind stress, $25 \%$ - by the wind with 10 days period.

Figure 32. Autocorrelation function of the fluctuating component of $v$ for experiment 29. There is a well-determined maximum at $x \approx 40.6 \mathrm{~km}$ corresponding to the most energetic scale of $v$.

Figure 33. The same as in Figure 31 but for experiments 30 with positive mean wind.

Figure 34. Map of the CODE region. (From Lentz, 1987.)

Figure A.1 BSW dispersion diagrams for the sheared background flow determined by equation (3.4), dashed lines with circles, and for the uniform (averaged in $y$ value of $U$ at some particular time) across the channel flow, solid lines: (a) the background flow profile and its $y$-average value are taken at $t=93.9$ days when $\bar{U}^{y}=9.8 \mathrm{~cm} / \mathrm{sec}$ (maximum, no-lee wave phase); (b) (a) the background flow profile and its $y$-average value are taken at $t=98.9$ days when $\bar{U}^{y}=-9.8 \mathrm{~cm} / \mathrm{sec}$ (minimum, lee wave phase). 
Figures 


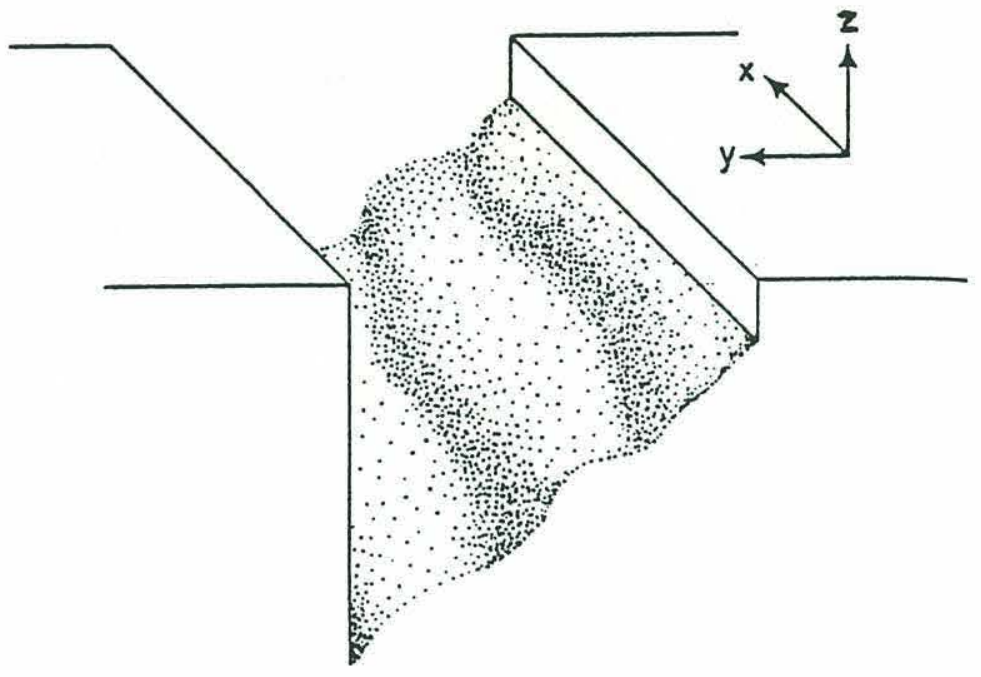

Figure 1. A sketch depicting the coastal channel geometry. (From Haidvogel and Brink, 1986.) 
(a)

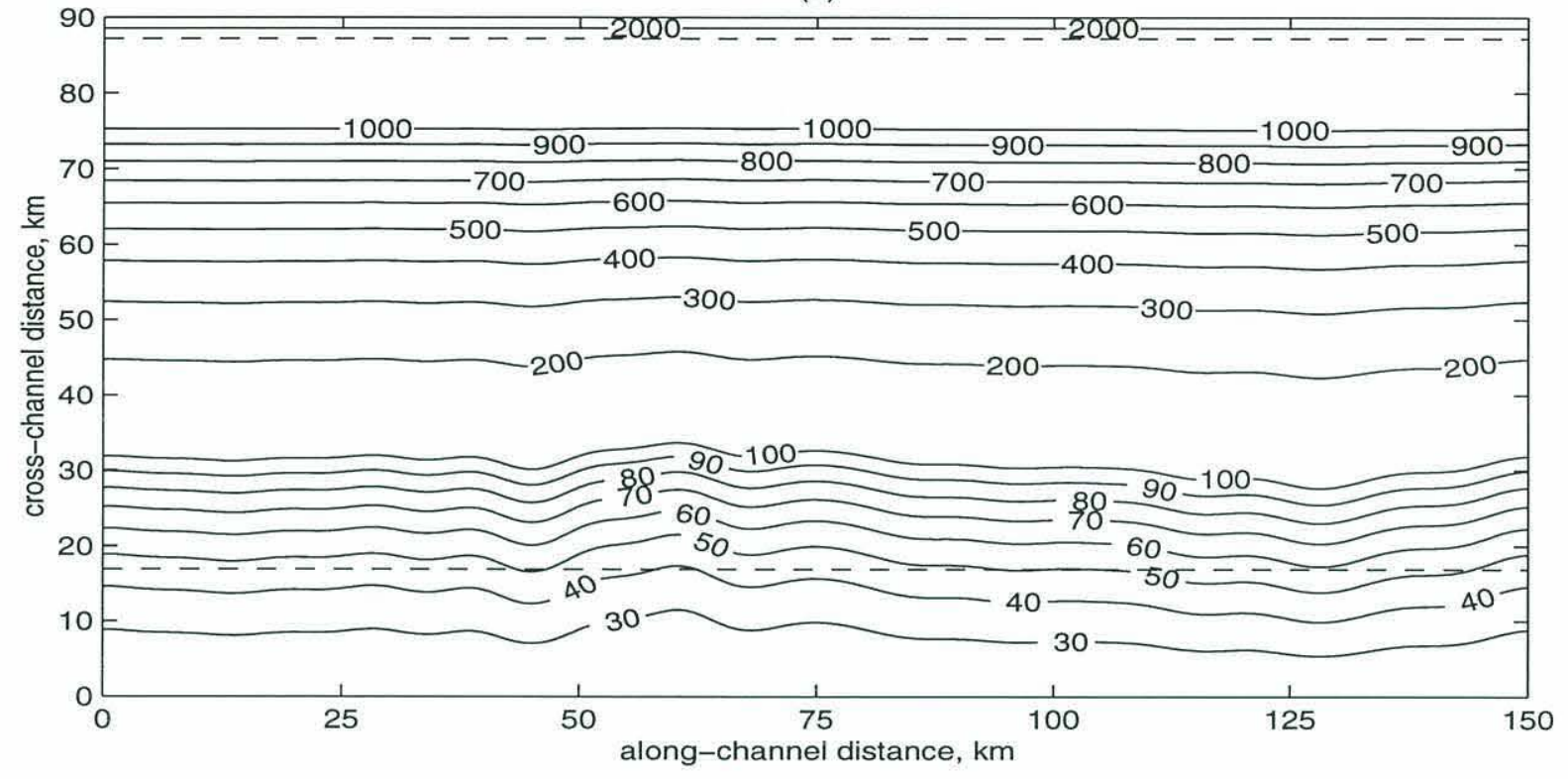

(b)

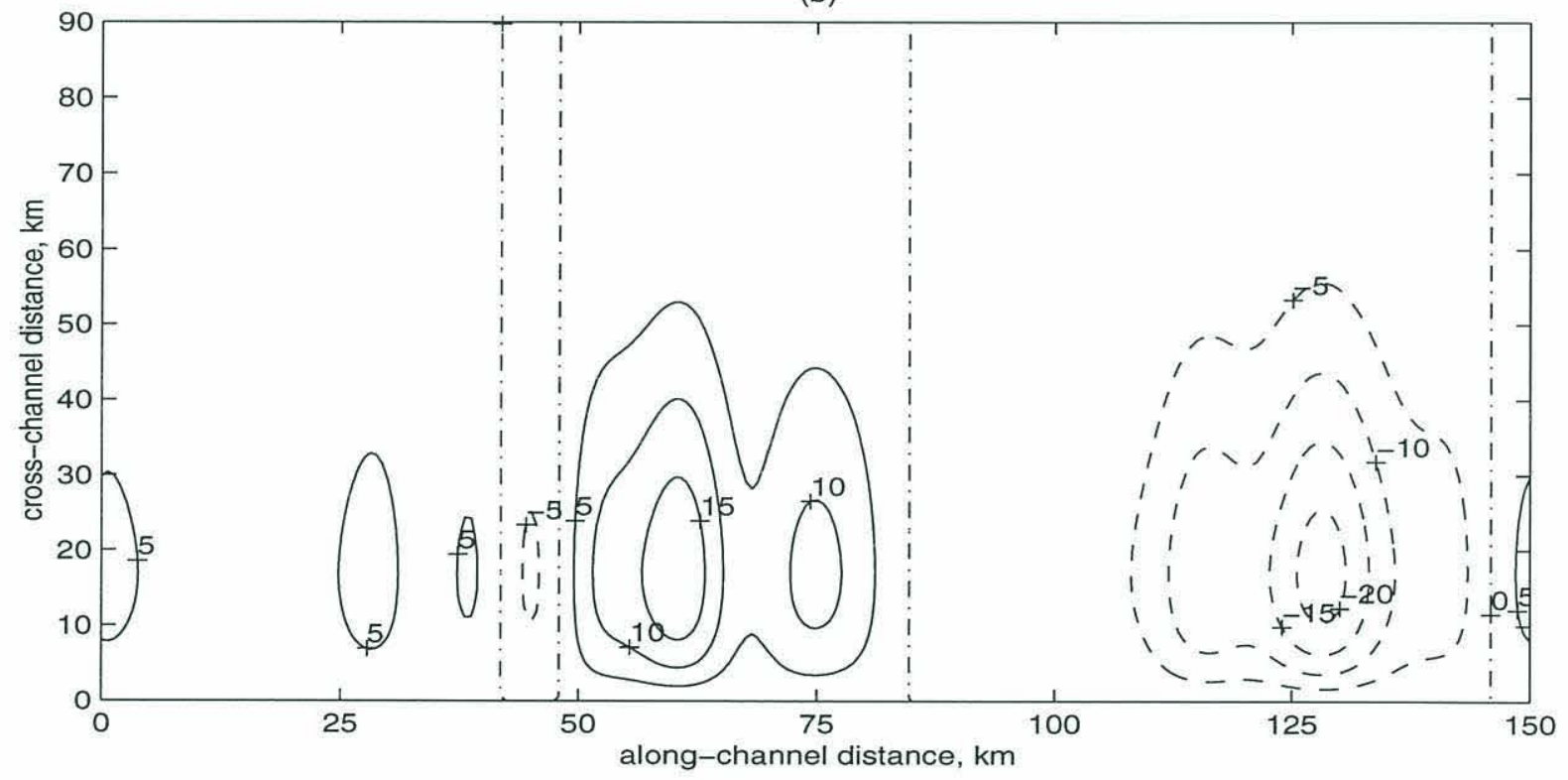

Figure 2: (a) Bottom topography $(m)$ in the channel with parameters corresponding to the Basic experiment (3). Dashed lines depict sections in the mid-shelf and near the deep boundary where data have been collected; (b) ratio of the topographic disturbance to the background depth (\%). 

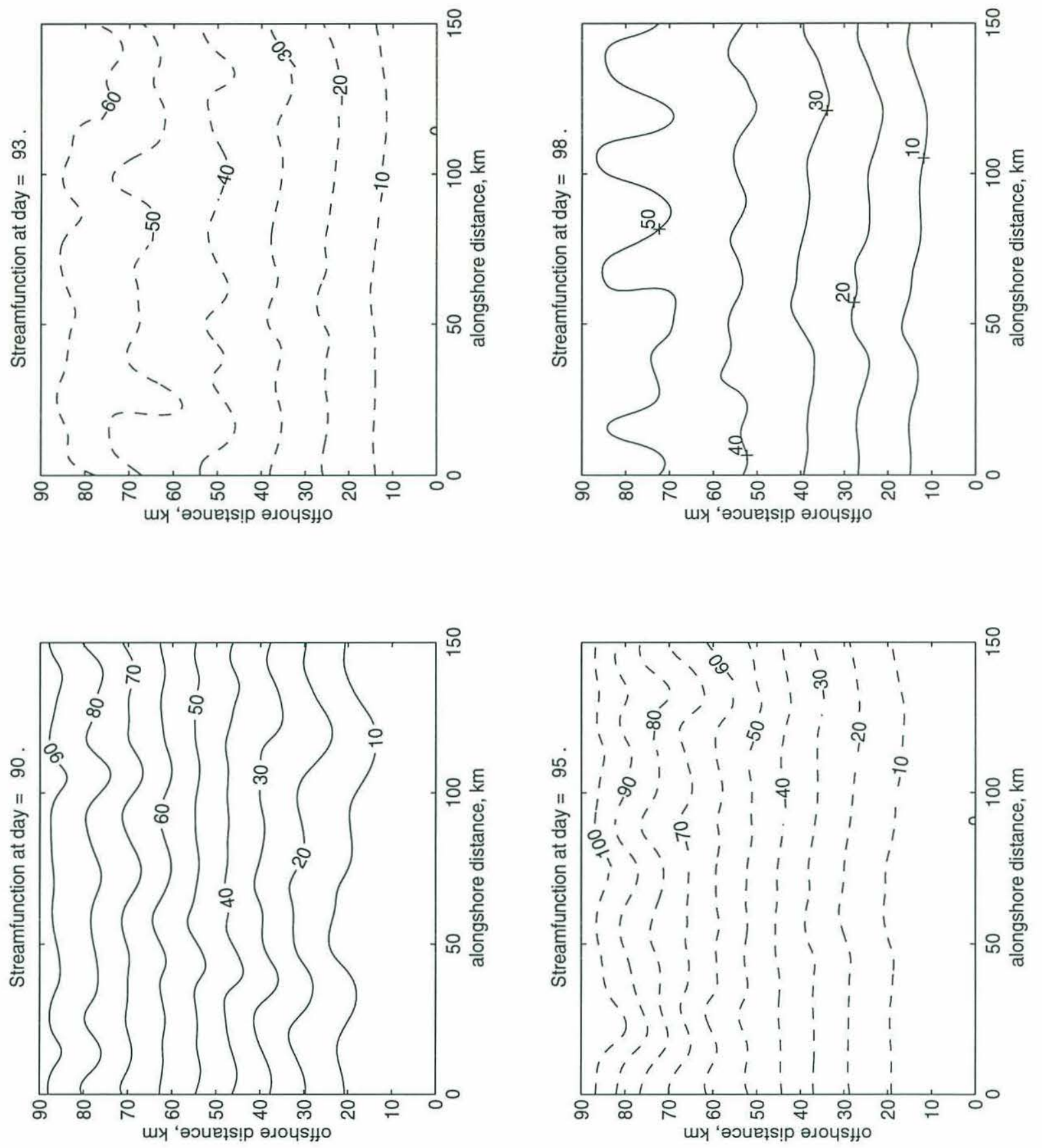

Figure 3: Evolution of the streamfunction field through a period of forcing: (a) $t=90$ days; (b) $t=93$ days; (c) $t=95$ days; (d) $t=98$ days. Solid lines correspond to positive values; dashed lines to negative values. (Basic experiment.) 


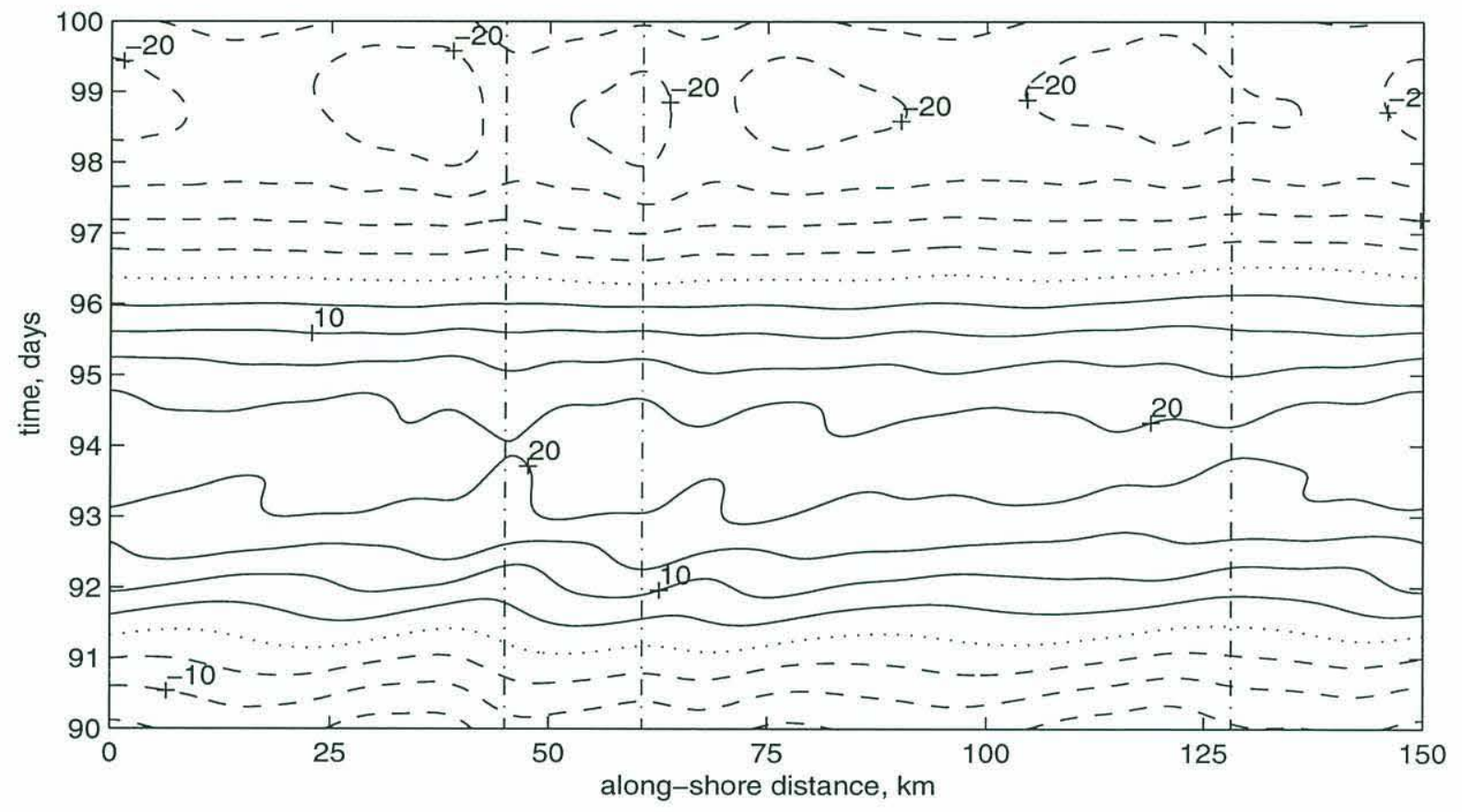

Figure 4: Alongshore flow on the mid-shelf section for a period of forcing $(\mathrm{cm} / \mathrm{sec})$. Solid lines correspond to positive values; dashed lines - to negative values; dotted lines - to $0 \mathrm{~cm} / \mathrm{sec}$; dash-dot lines - to axes of the most pronounced topographic ridges or canyons. The same is true for other $t-x$ diagrams, unless otherwise stated. (Basic experiment.) 


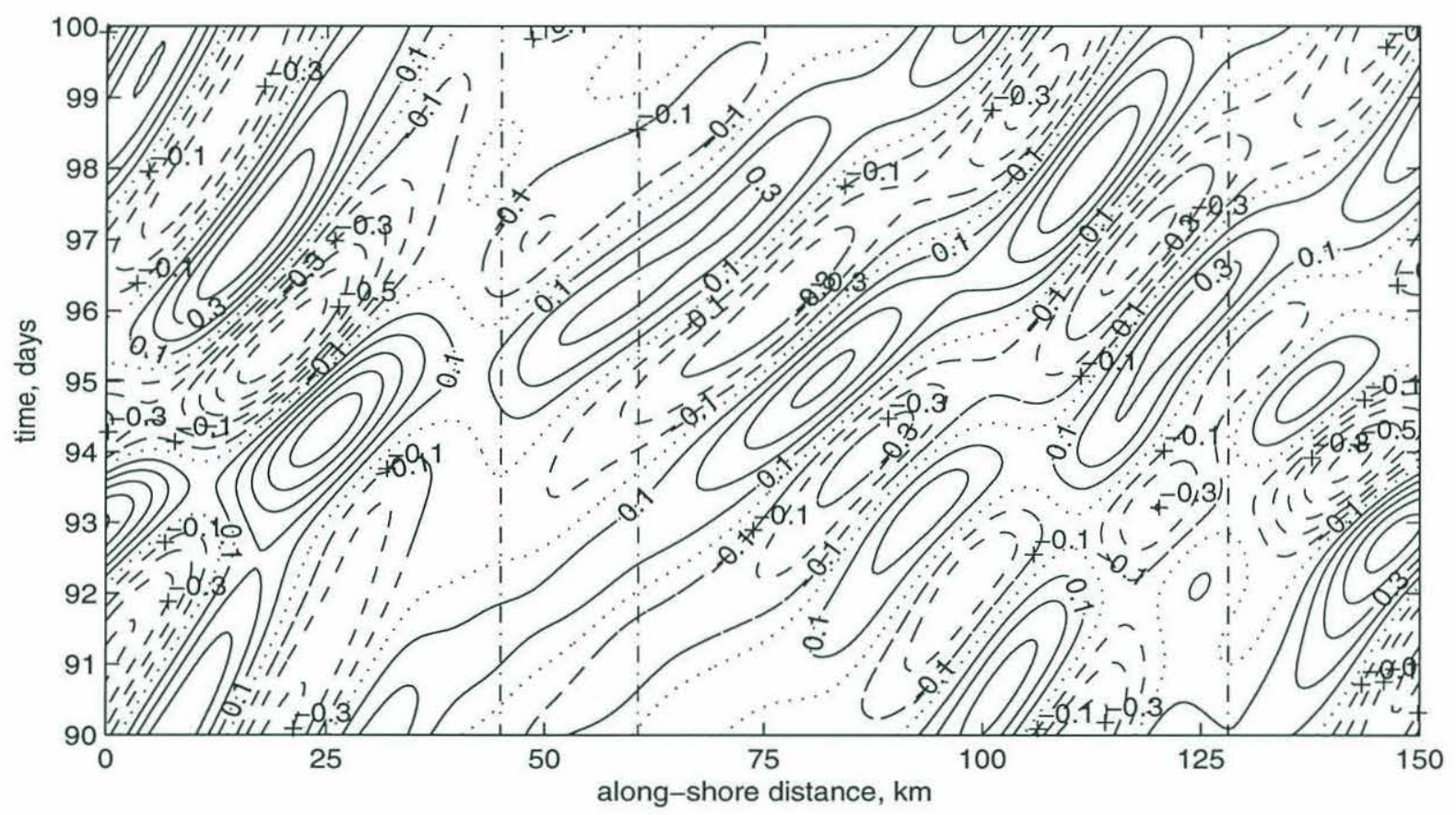

Figure 5: Cross-channel flow $(\mathrm{cm} / \mathrm{sec})$ in the deep part of the channel, depth about $2000 \mathrm{~m}$. (Basic experiment.) 
(a)

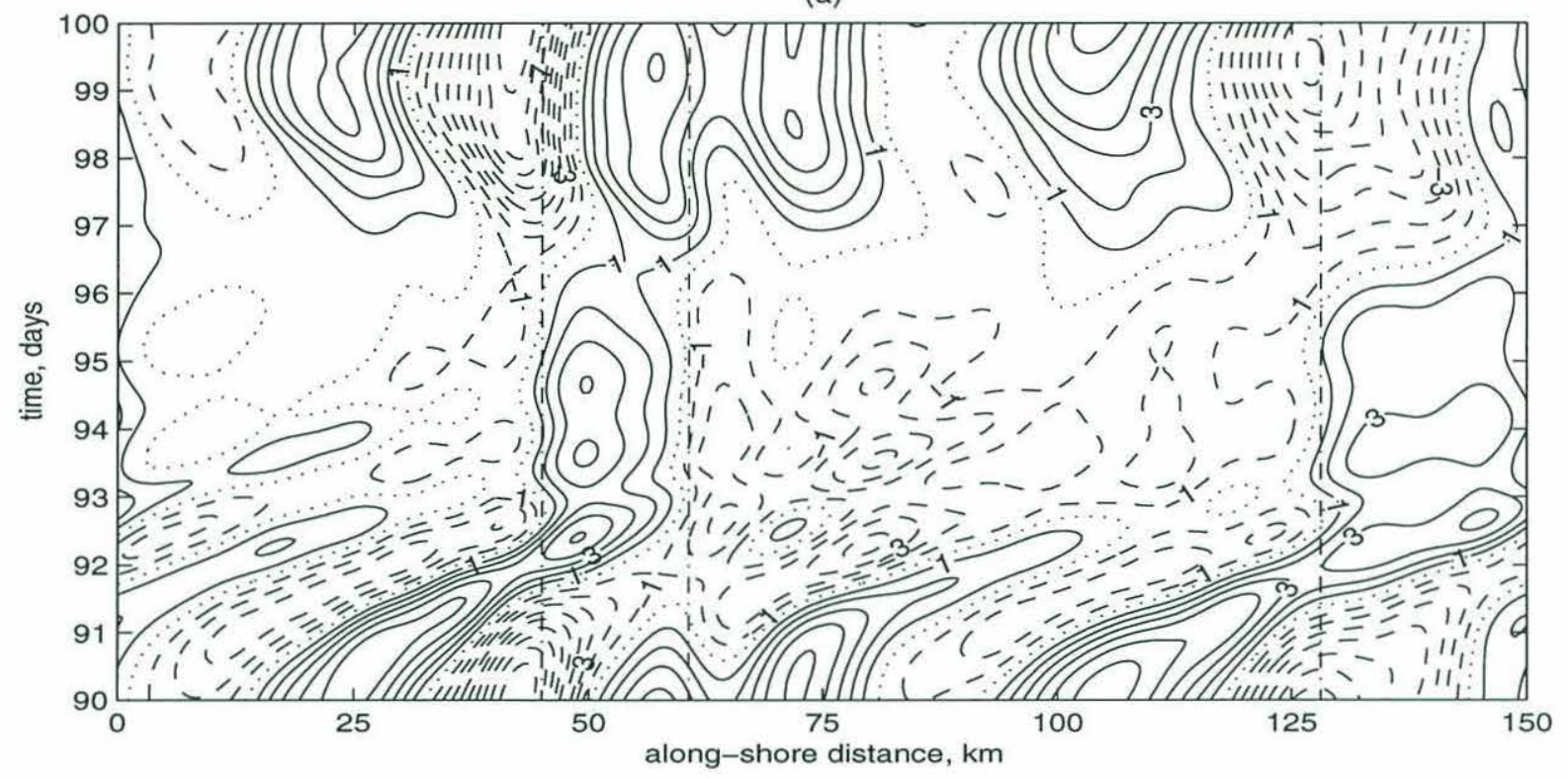

(b)

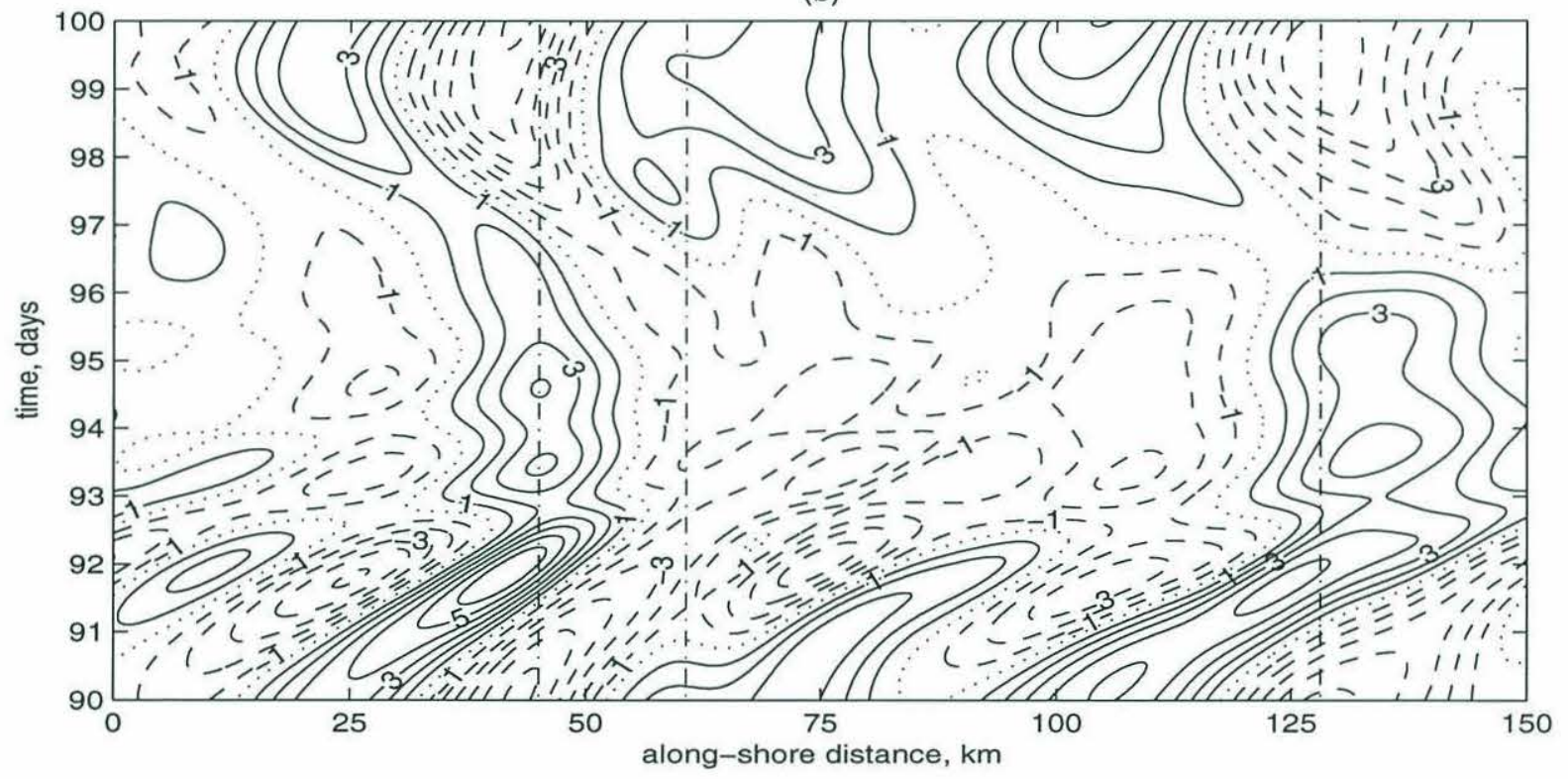

Figure 6: Flow components $(\mathrm{cm} / \mathrm{sec})$ on the mid-shelf section at $x=50 \mathrm{~km}$ : (a) cross-channel current; (b) along-channel current. Dashed line corresponds to the background current. (Basic experiment.) 
(a)

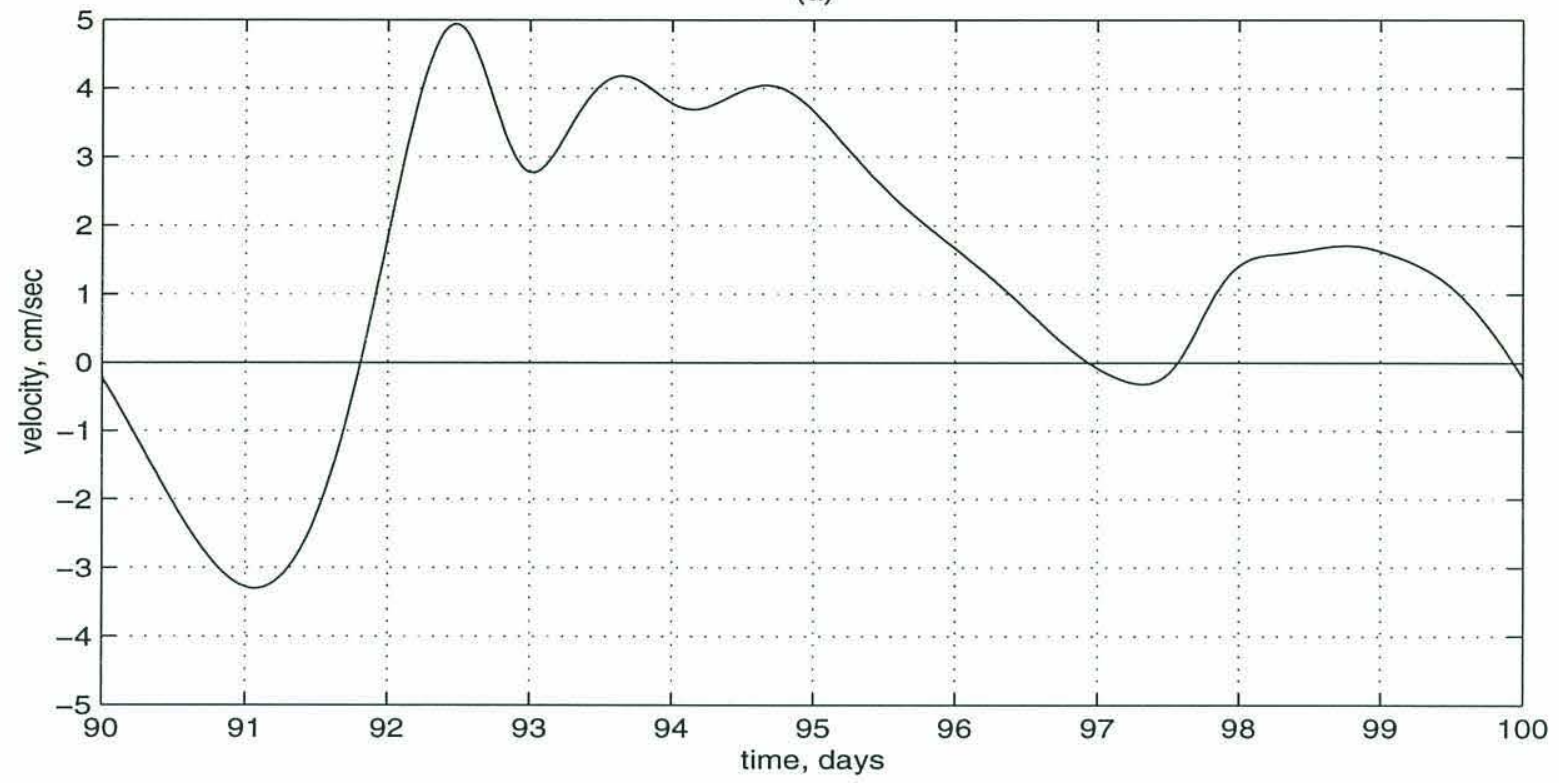

(b)

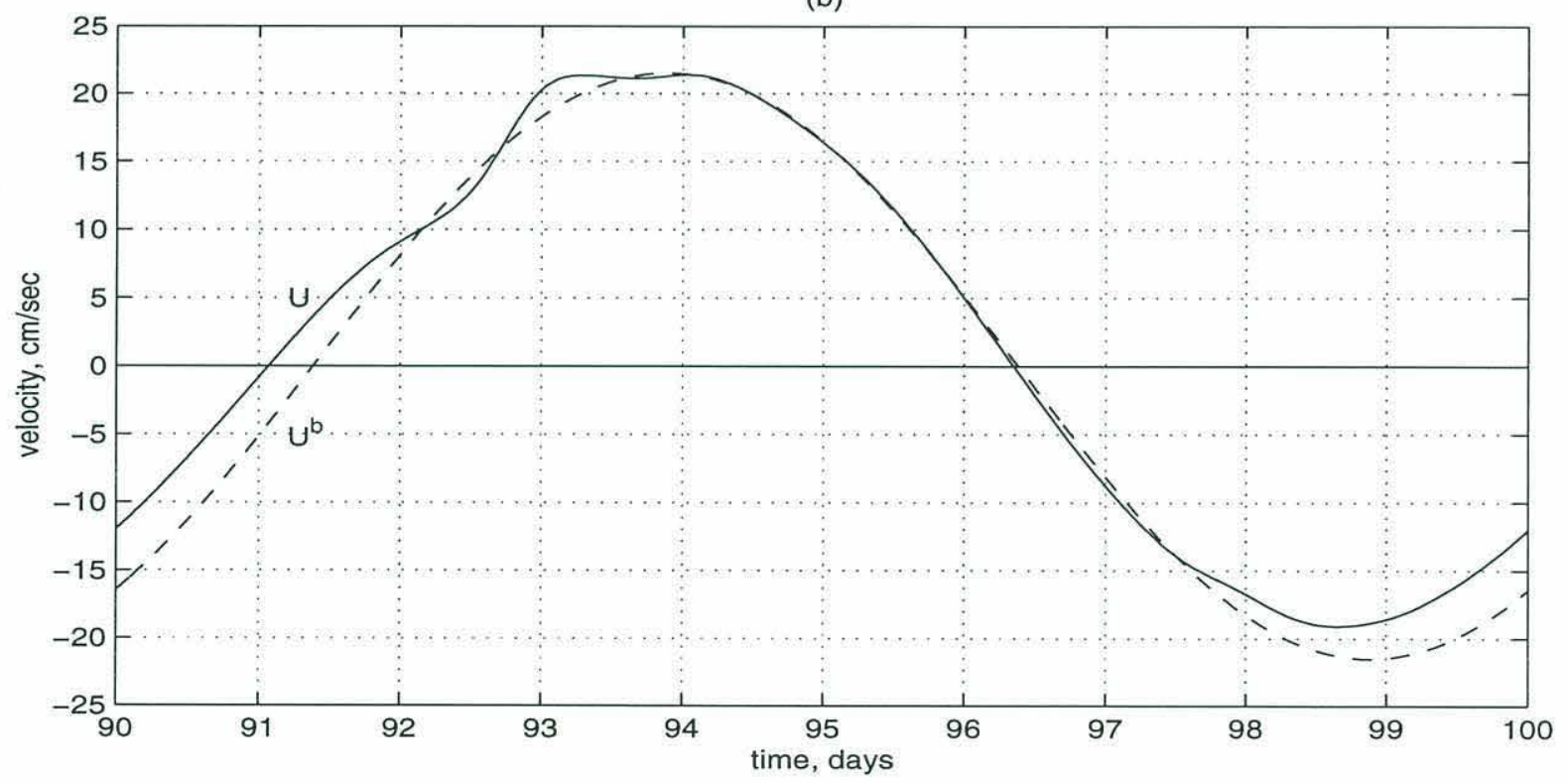

Figure 7: Flow components $(\mathrm{cm} / \mathrm{sec})$ on the mid-shelf section at $x=50 \mathrm{~km}$ : (a) cross-channel current; (b) along-channel current. Dashed line corresponds to the background current. (Basic experiment.) 
(a)

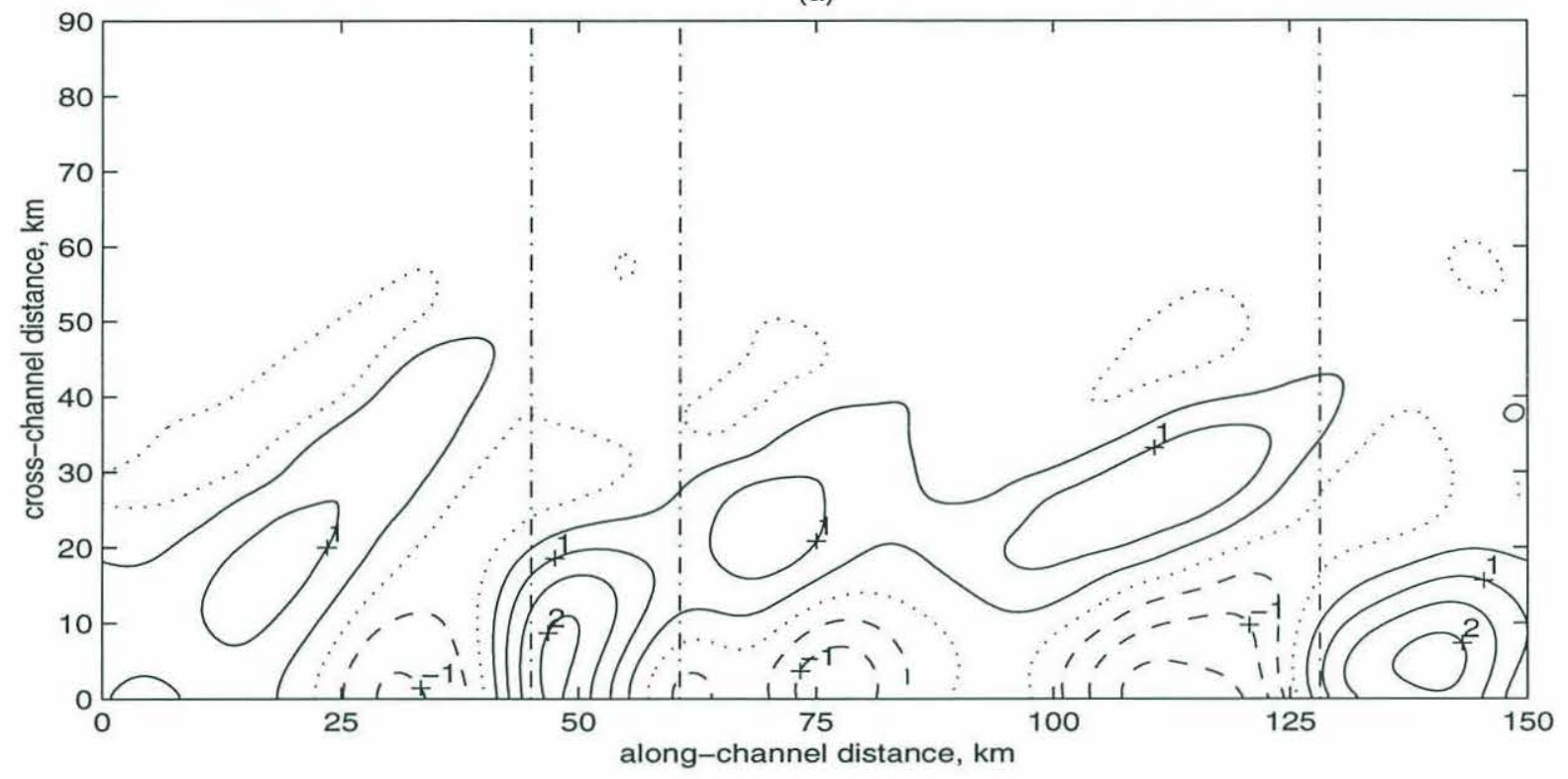

(b)

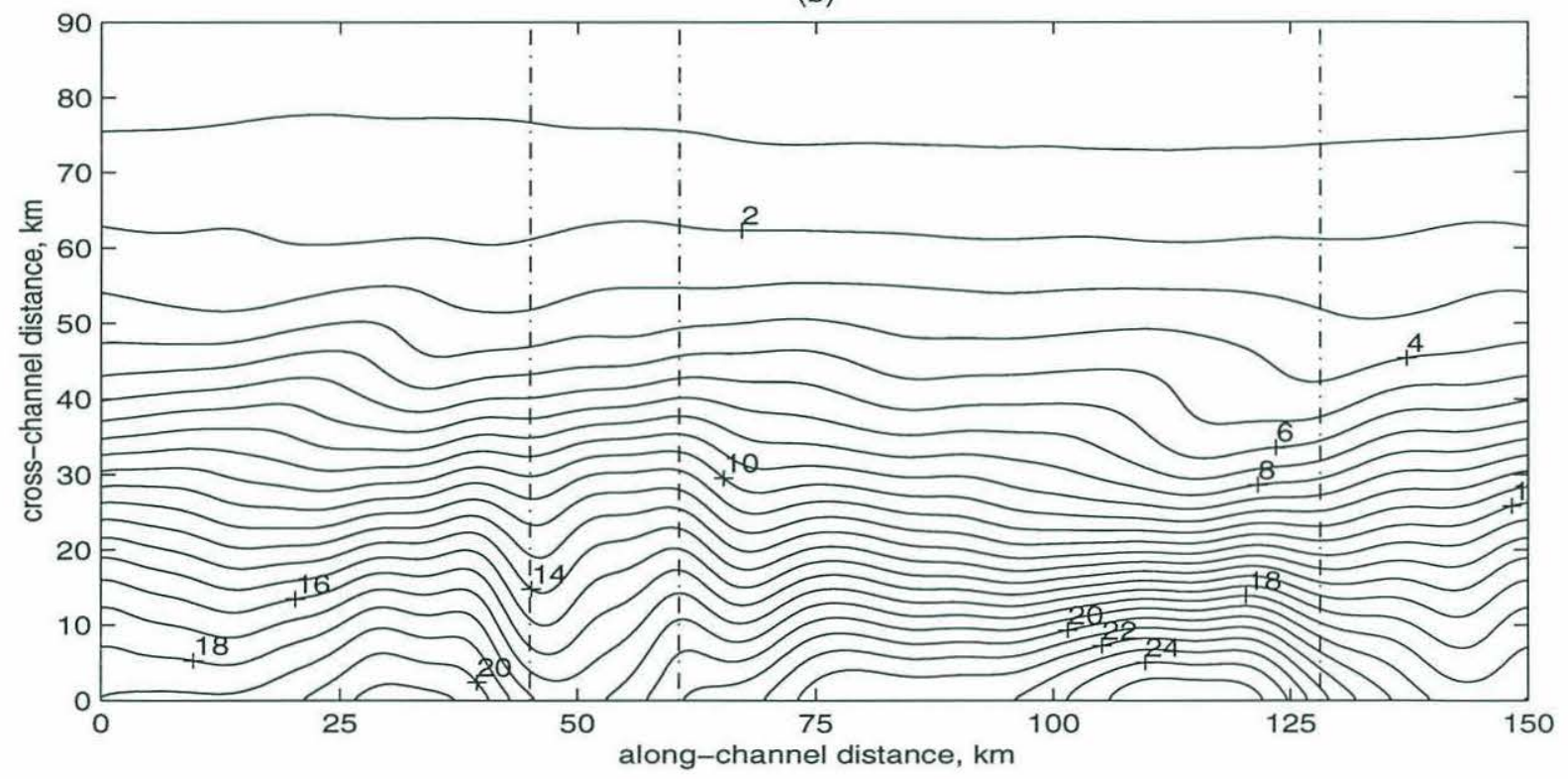

Figure 8: (a) Time-mean alongshore currents $(\mathrm{cm} / \mathrm{sec}$ ); (b) standard deviation of alongshore currents ( $\mathrm{cm} / \mathrm{sec}$ ). (Basic experiment.) 


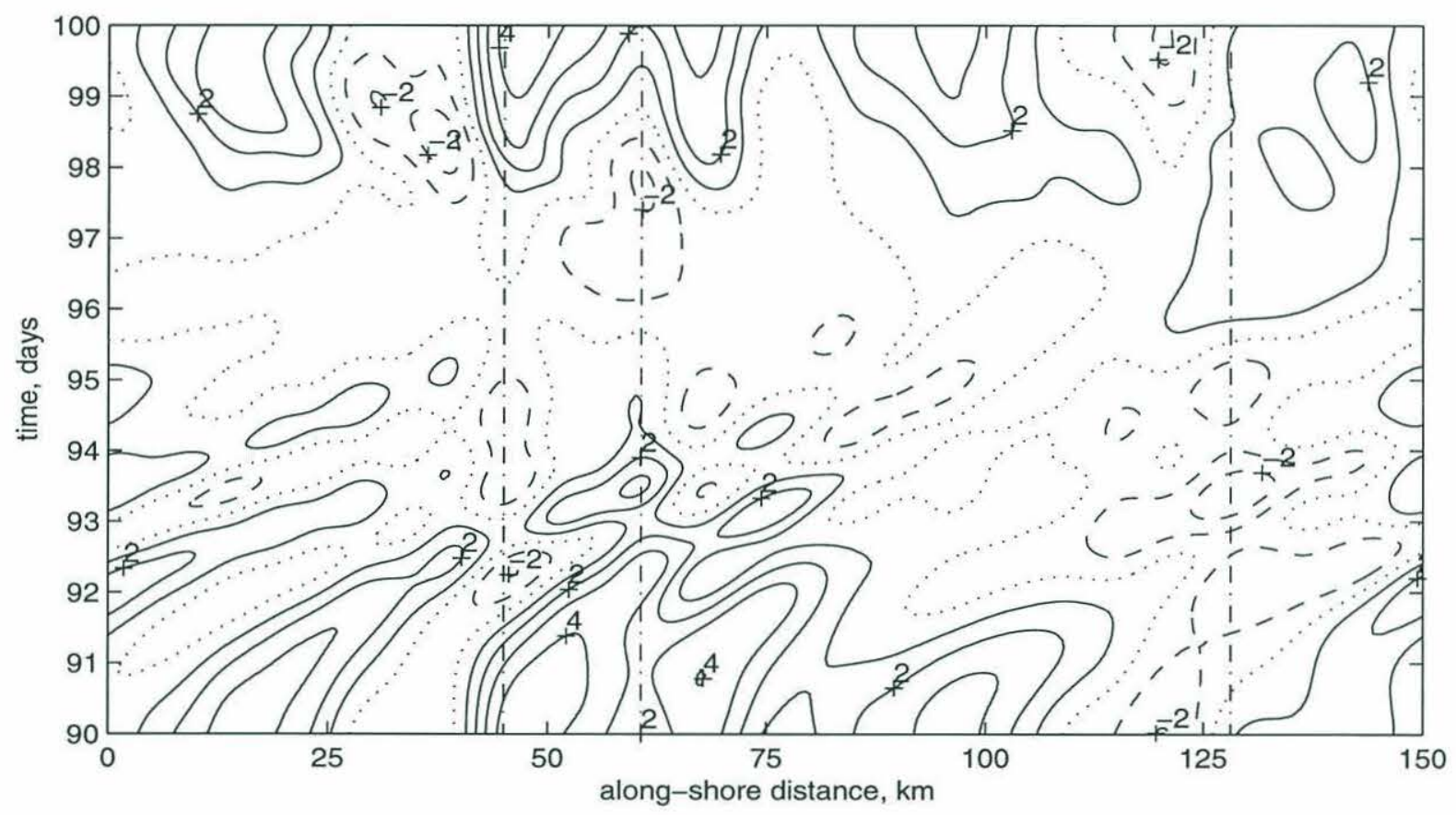

Figure 9: Difference between the total alongshore flow and the background current $(\mathrm{cm} / \mathrm{sec})$ in the middle of the shelf. (Basic experiment.) 
(a) Terms of the time and along-channel averaged $x-$ momentum balance.

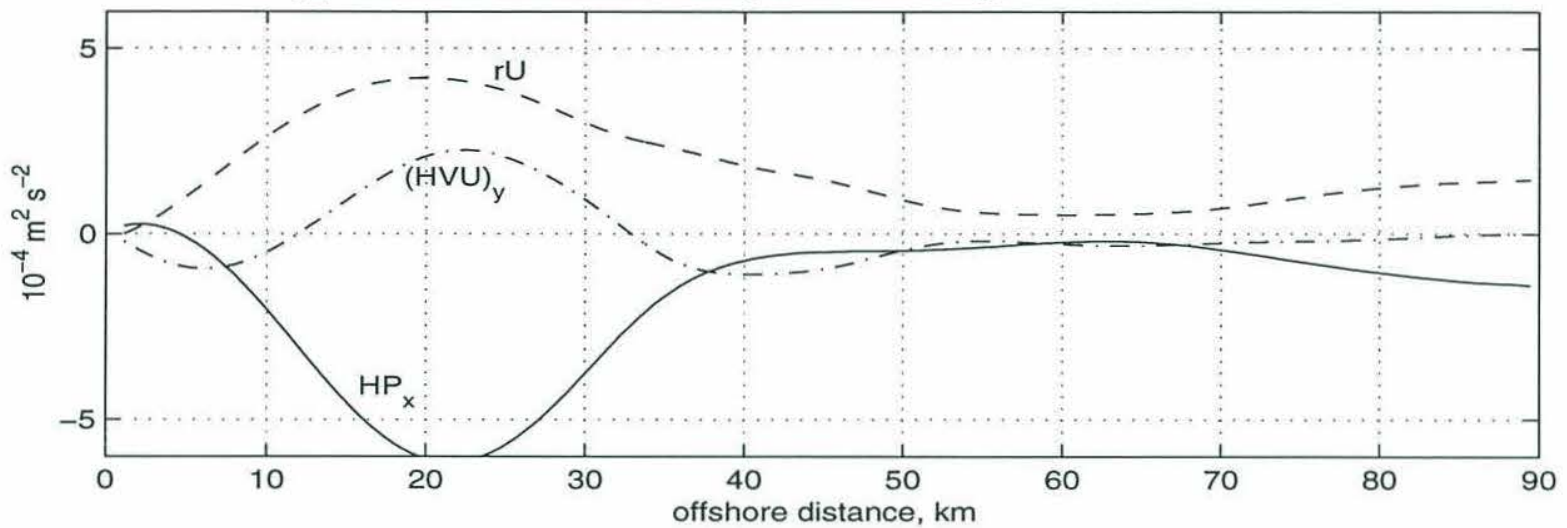

(b) $\mathrm{x}$-averaged $\mathrm{HP}_{\mathrm{x}}\left(10^{-3} \mathrm{~m}^{2} \mathrm{~s}^{-2}\right)$.

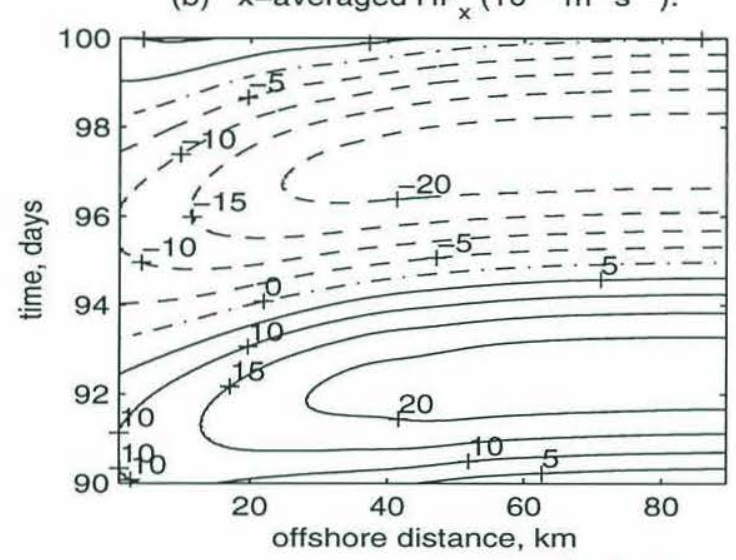

(c) $\mathrm{x}$-averaged $\mathrm{rU}\left(10^{-3} \mathrm{~m}^{2} \mathrm{~s}^{-2}\right)$.

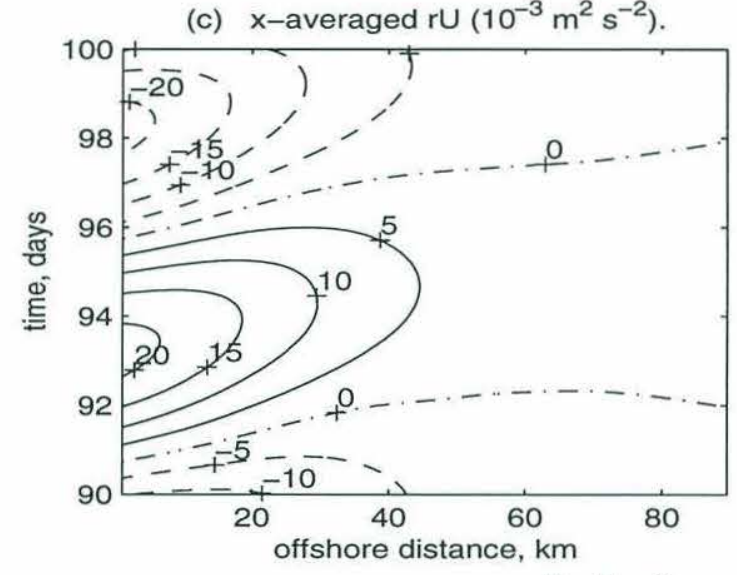

(d) $\mathrm{x}$-averaged $(\mathrm{HUV})_{\mathrm{y}}\left(10^{-3} \mathrm{~m}^{2} \mathrm{~s}^{-2}\right)$.

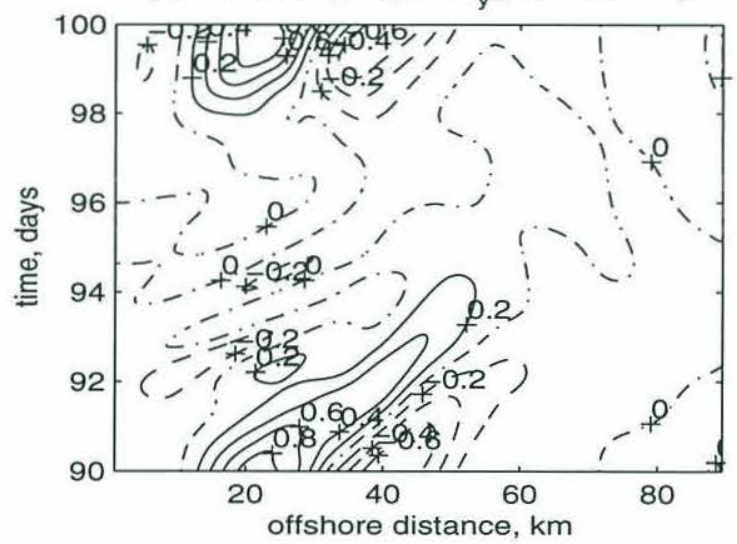

(e) $\mathrm{x}$-averaged $(\mathrm{HU})_{\mathrm{t}}\left(10^{-3} \mathrm{~m}^{2} \mathrm{~s}^{-2}\right)$.

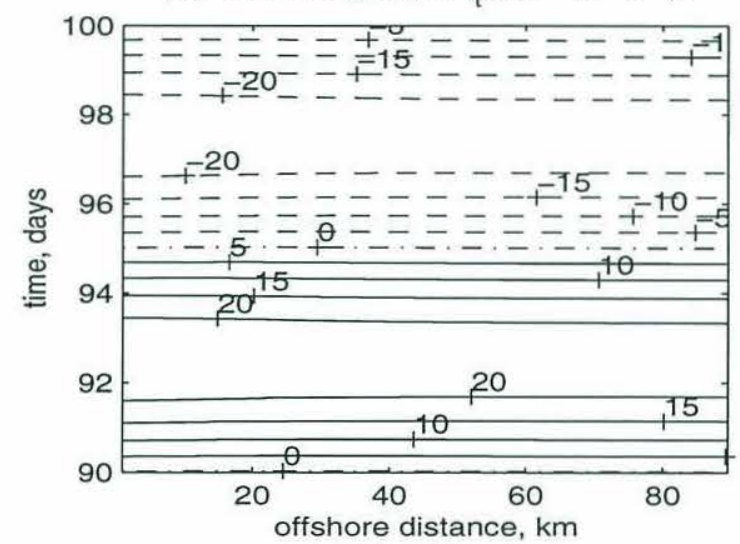

Figure 10: (a) Components of averaged (over a period and over the channel) $x$-momentum balance $\left(10^{-4} \mathrm{~m}^{2} / \mathrm{sec}^{2}\right)$. Terms of the averaged along the channel $x$-momentum balance $\left(10^{-3} \mathrm{~m}^{2} / \mathrm{sec}^{2}\right)$ : (b) ${\overline{H \phi_{x}}}^{x}$, (c) $r \bar{u}^{x}$, (d) $\frac{\partial}{\partial y} \overline{(H u v)}^{x}$, (e) ${\overline{(H u)_{t}}}^{x}$. (Basic experiment.) 
(a)

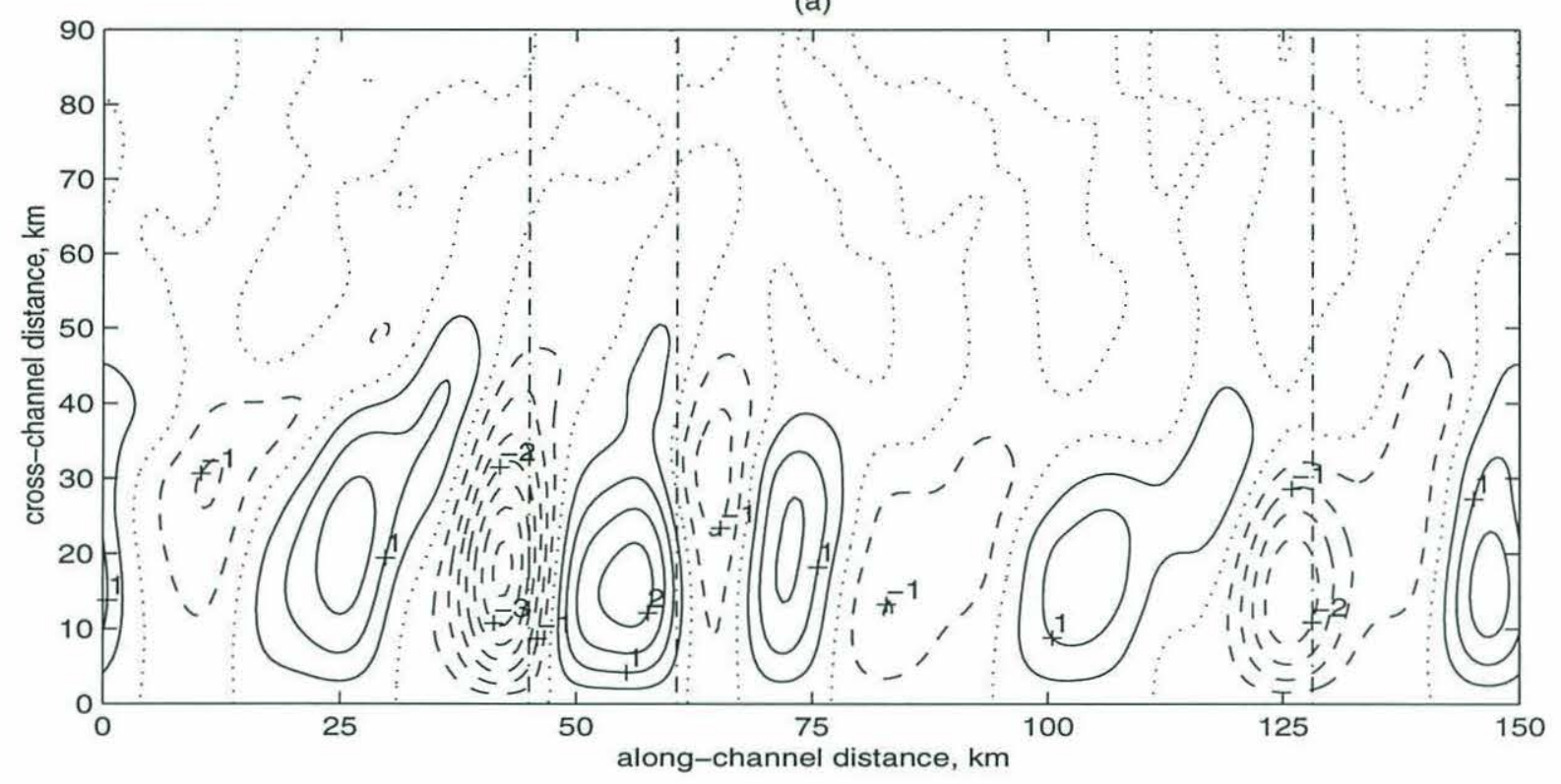

(b)

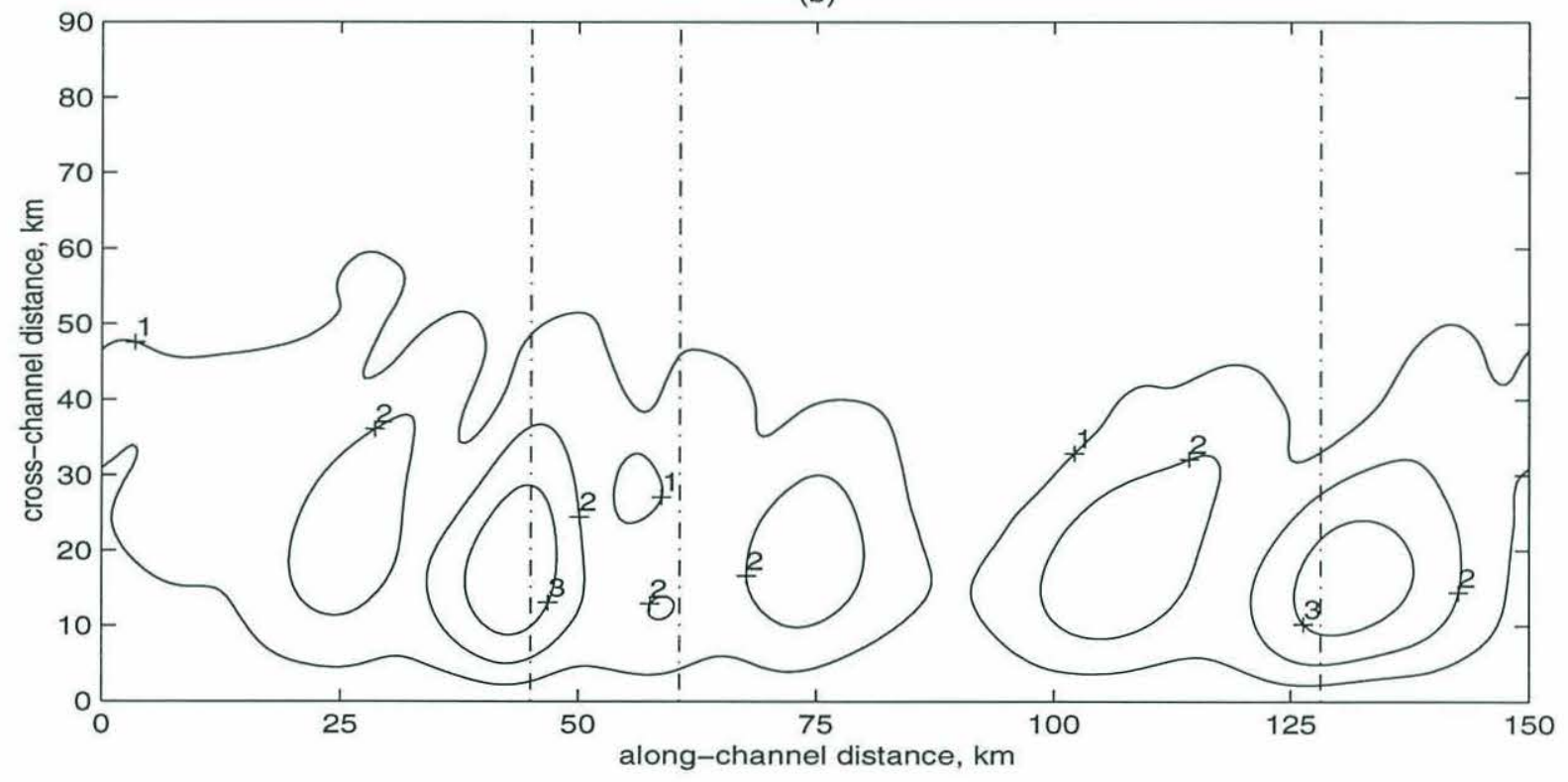

Figure 11: (a) Mean over a period offshore currents $(\mathrm{cm} / \mathrm{sec})$; (b) standard deviation of cross-channel currents $(\mathrm{cm} / \mathrm{sec})$. (Basic experiment.) 
(a)

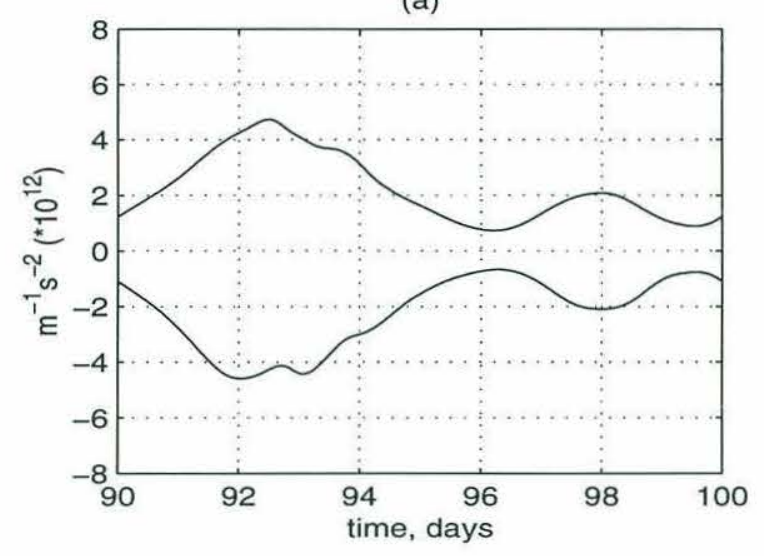

(c)

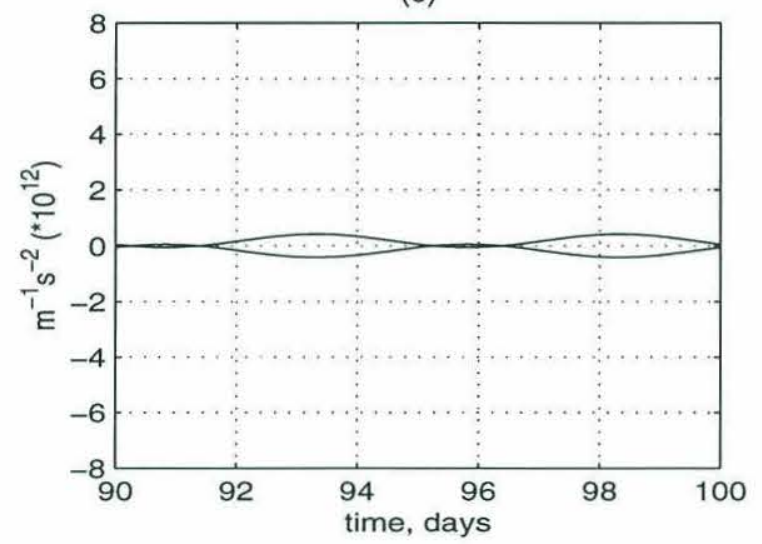

(e)

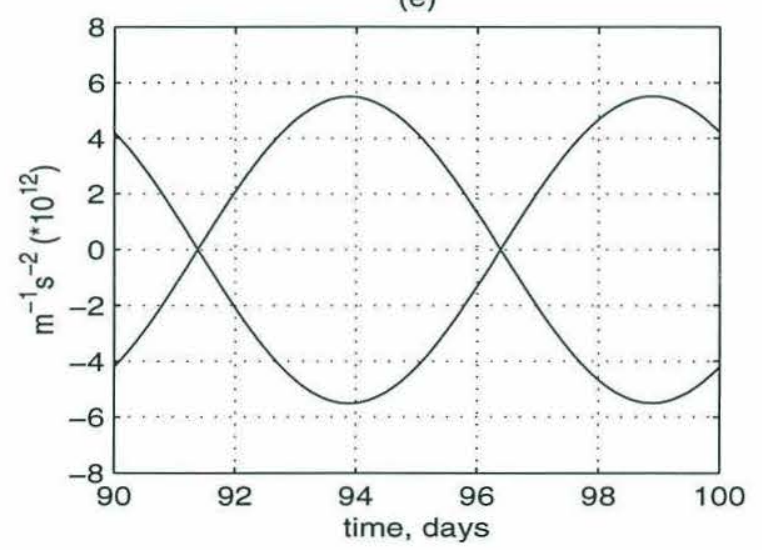

(b)

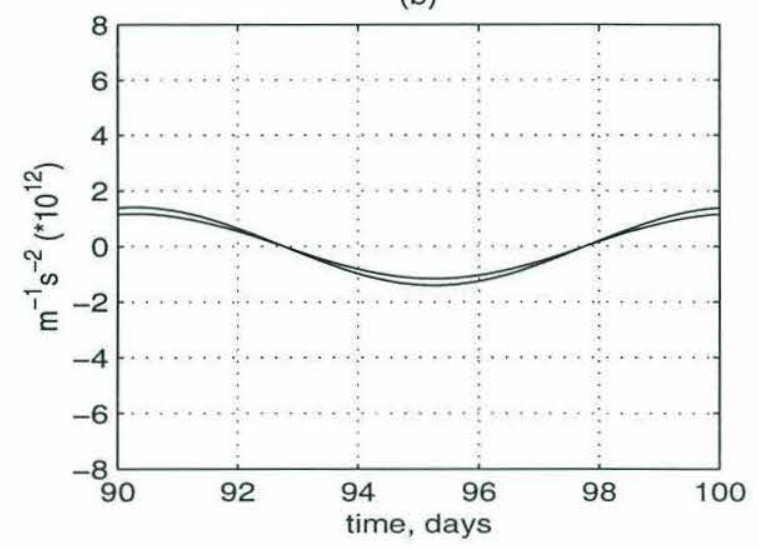

(d)

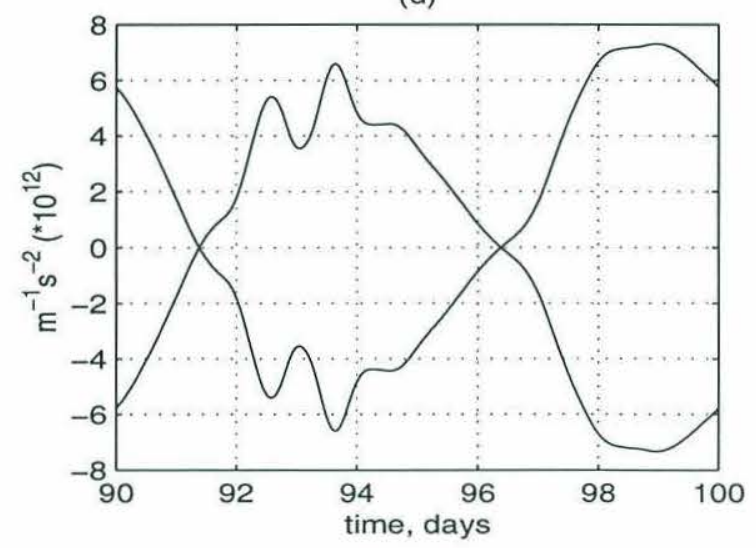

(f)

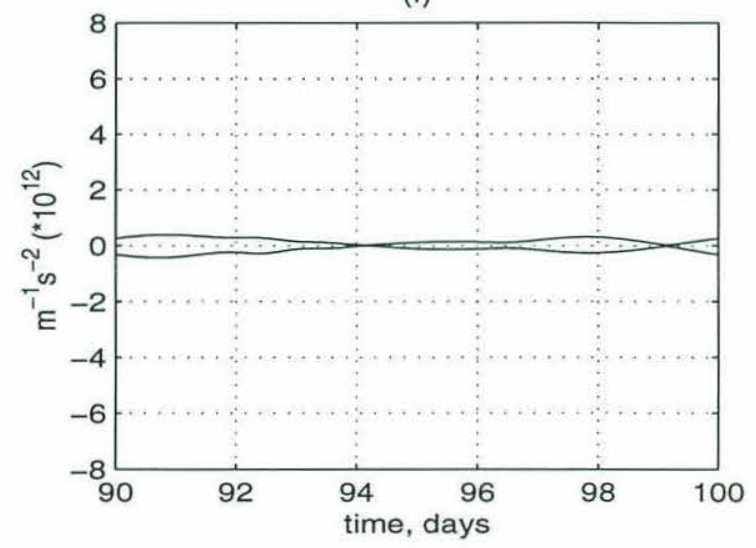

Figure 13: Mean (over the channel length) with a range given by \pm standard deviation terms in the vorticity balance (3.7) at the mid-shelf section for one period of forcing: (a) $\partial_{t}\left(\frac{\zeta^{\prime}}{H}\right),(\mathrm{b}) \partial_{t}\left(\frac{\zeta}{H}\right),(\mathrm{c}) U \partial_{x}\left(\frac{\zeta}{H}\right)$, (d) $U \partial_{x}\left(\frac{\zeta^{\prime}}{H}\right),\left(\right.$ e) $U \partial_{x}\left(\frac{f}{H}\right),($ f $)(\vec{u} \cdot \nabla) \frac{\zeta}{H}$. (Basic experiment.) 
(a)

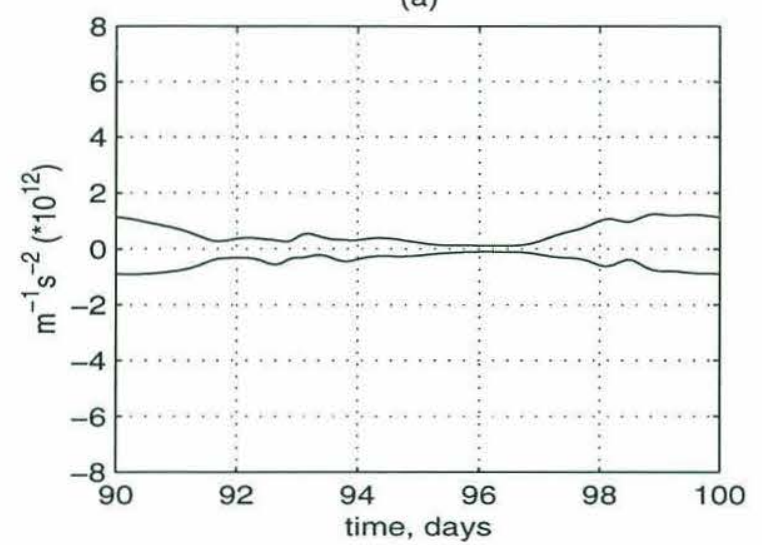

(c)

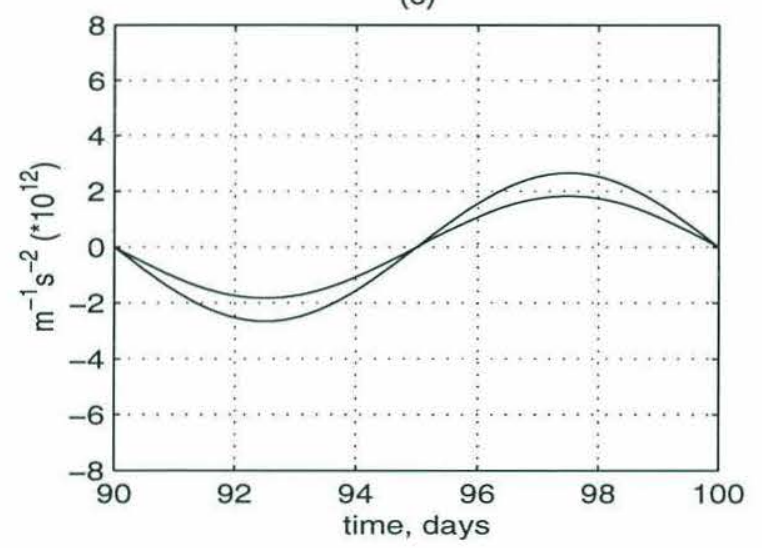

(e)

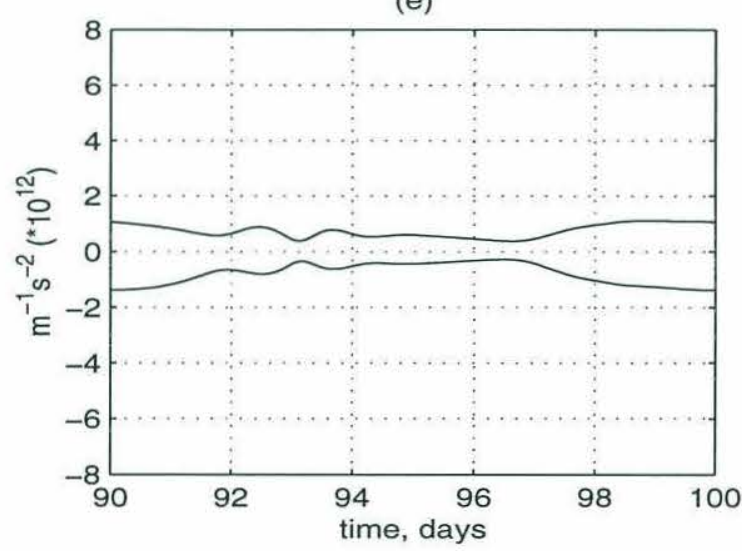

(b)

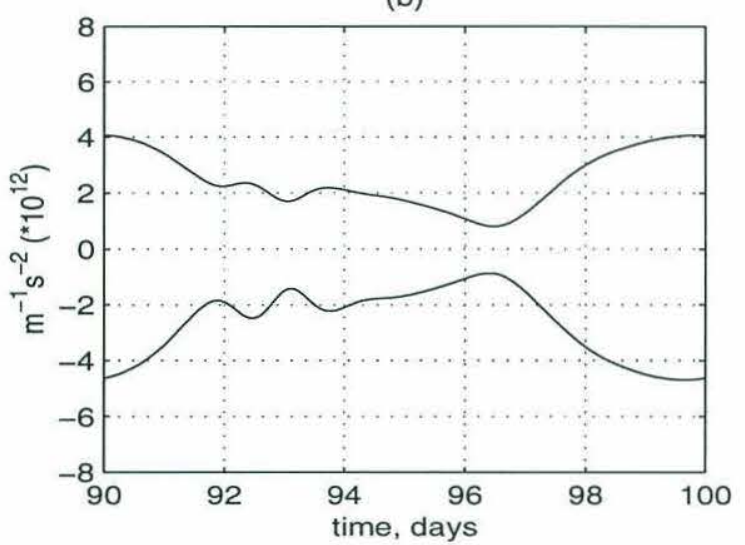

(d)

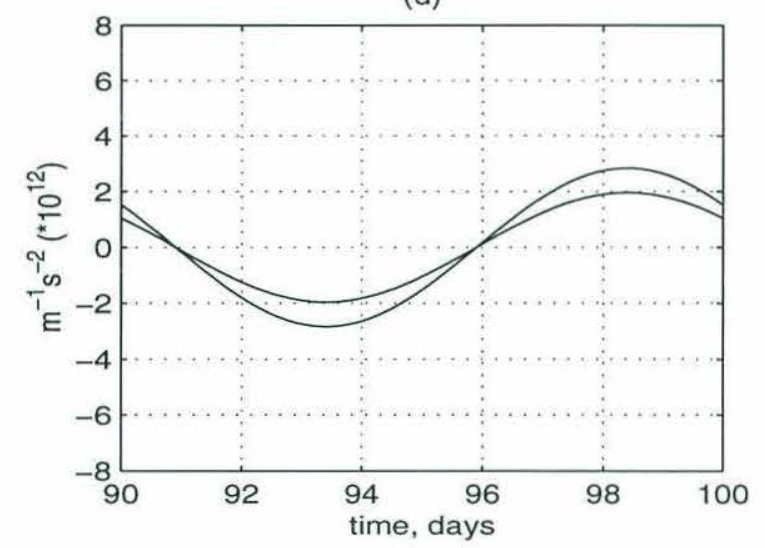

(f)

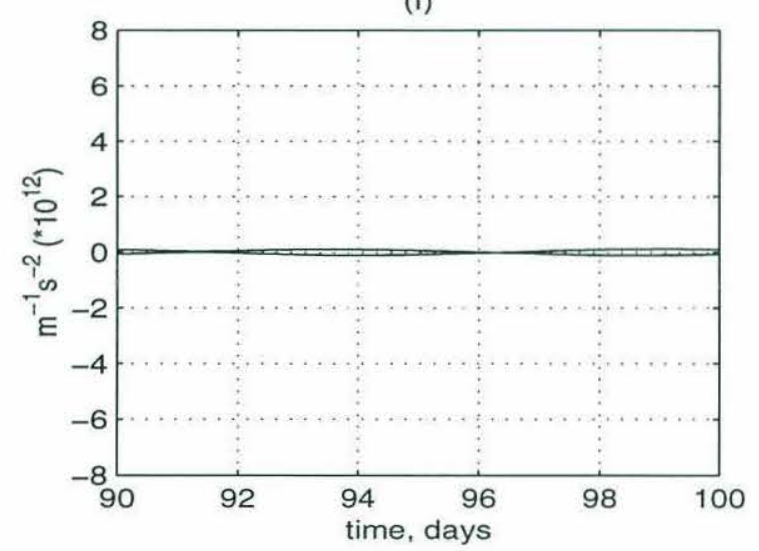

Figure 14: The same as in Figure 13 but for (a) $(\vec{u} \cdot \nabla) \frac{\zeta^{\prime}}{H}$, (b) $(\vec{u} \cdot \nabla) \frac{f}{H}$, (c) $\frac{\tau^{\alpha}}{H} \partial_{y}\left(\frac{1}{H}\right)$, (d) $\frac{r}{H} \partial_{y}\left(\frac{U}{H}\right),($ e $) \frac{r}{H}(\nabla \times$ $\left.\frac{\vec{u}}{H}\right)$, (f)imbalance. 
(a)

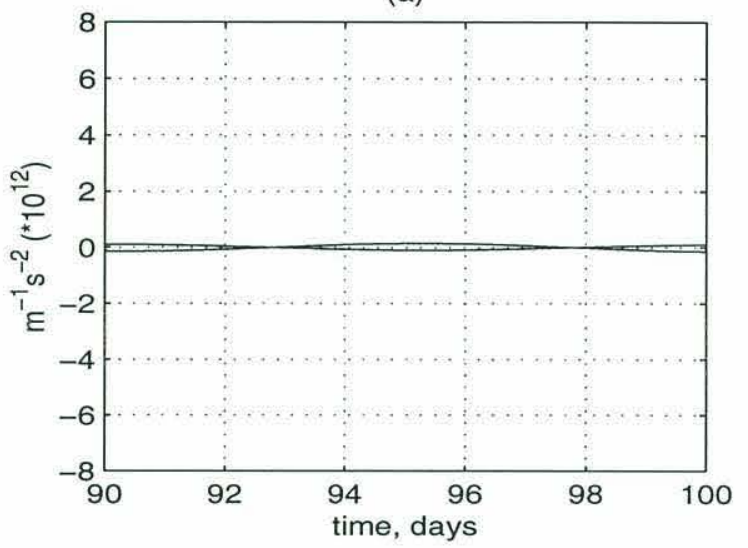

(b)

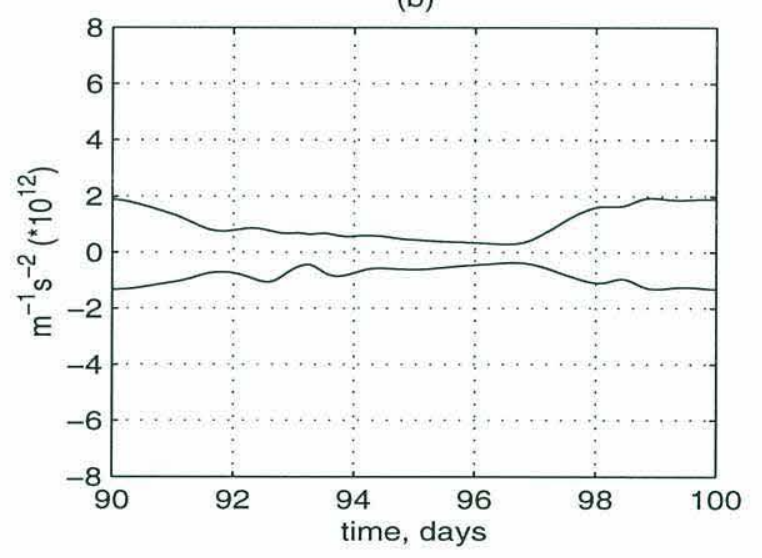

(c)

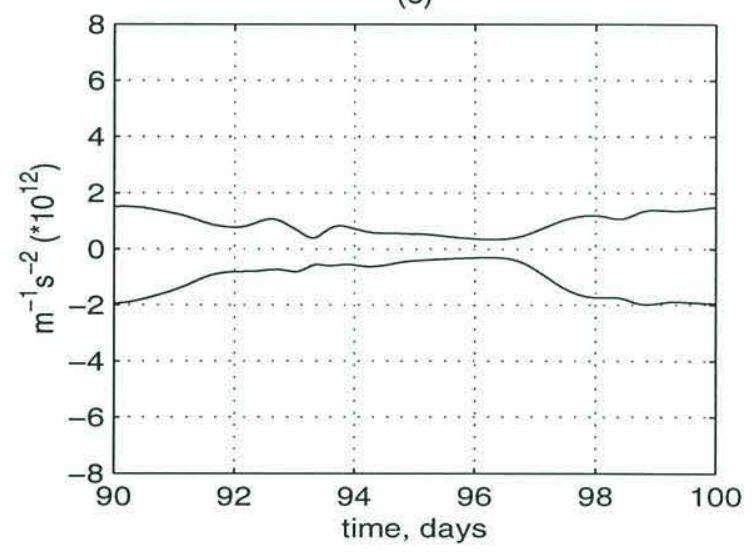

Figure 15: The same as in Figure 13 but for different physically meaningful combinations of terms: (a)the background current balance (3.10), (b)the balance associated with linear, non-viscous interaction of the background current with perturbation flow and topographic disturbances: $\left(\partial_{t}+U \partial_{x}\right)\left(\frac{\zeta^{\prime}}{H}\right)+(\vec{u} \cdot \nabla) \frac{f}{H}=$ $-U \partial_{x}\left(\frac{f}{H}\right),(\mathrm{c})$ sum of non-linear term and friction due to perturbation: $(\vec{u} \cdot \nabla) \frac{\zeta^{\prime}}{H}+\frac{r}{H}\left(\nabla \times \frac{\vec{u}}{H}\right)$. 
(a)

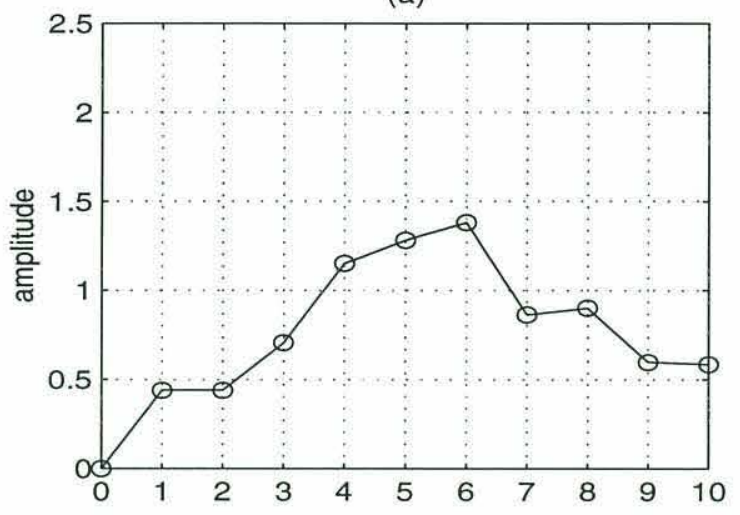

(c)

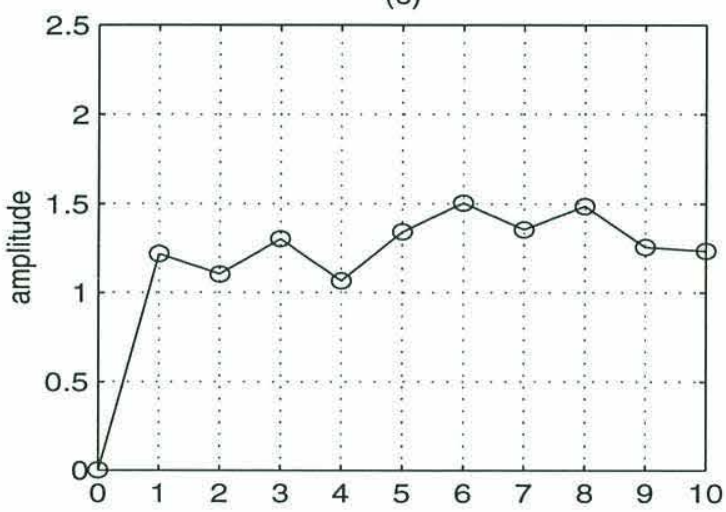

(e)

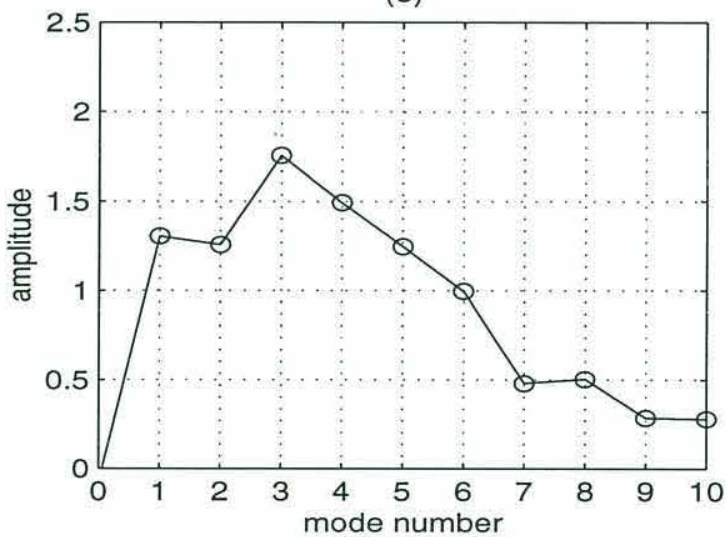

(b)

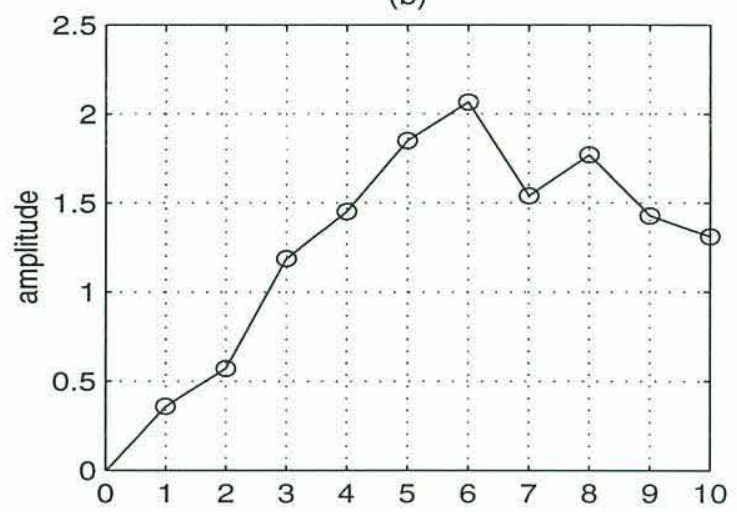

(d)

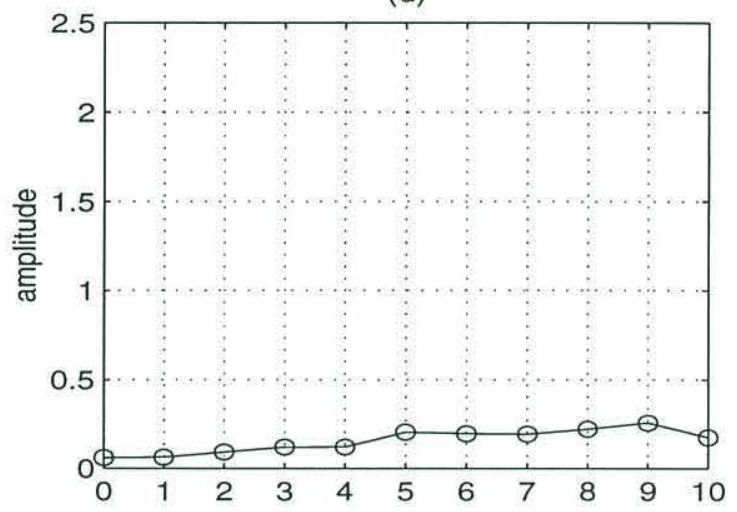

(f)

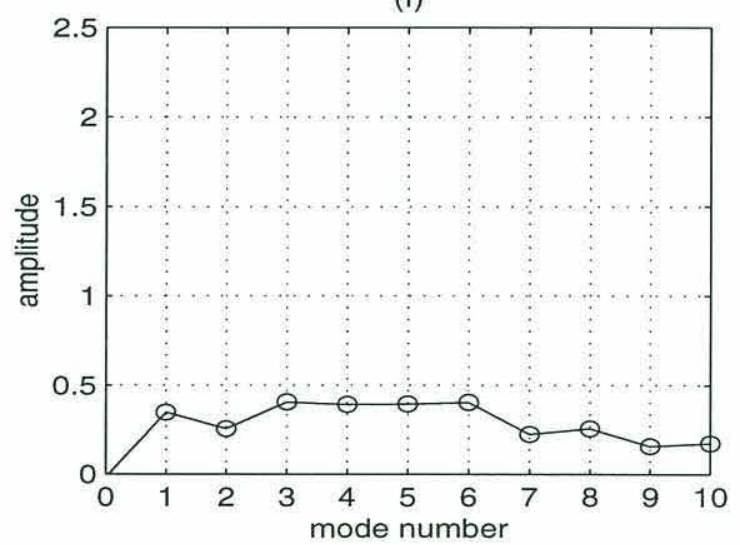

Figure 16: Time-mean amplitudes of alongshore Fourier components of terms in vorticity balance (3.11): (a) $\partial_{t}\left(\frac{\zeta^{\prime}}{H}\right),(\mathrm{b}) U \partial_{x}\left(\frac{\zeta^{\prime}}{H}\right),(\mathrm{c}) U \partial_{x}\left(\frac{f}{H}\right),(\mathrm{d})(\vec{u} \cdot \nabla) \frac{\zeta^{\prime}}{H},(\mathrm{e})(\vec{u} \cdot \nabla) \frac{f}{H},(\mathrm{f}) \frac{r}{H}\left(\nabla \times \frac{\vec{u}}{H}\right)$. 
(a)

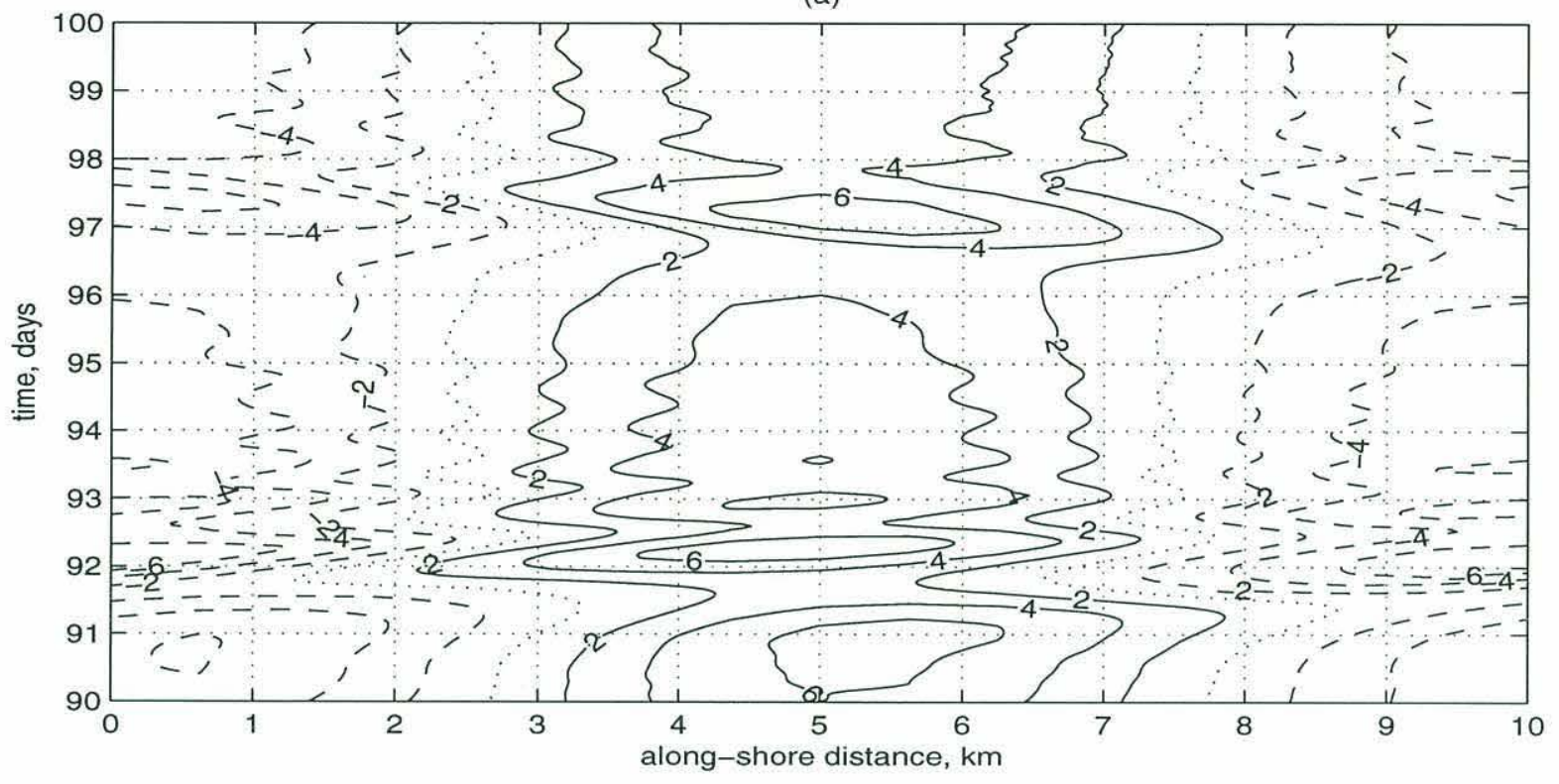

(b)

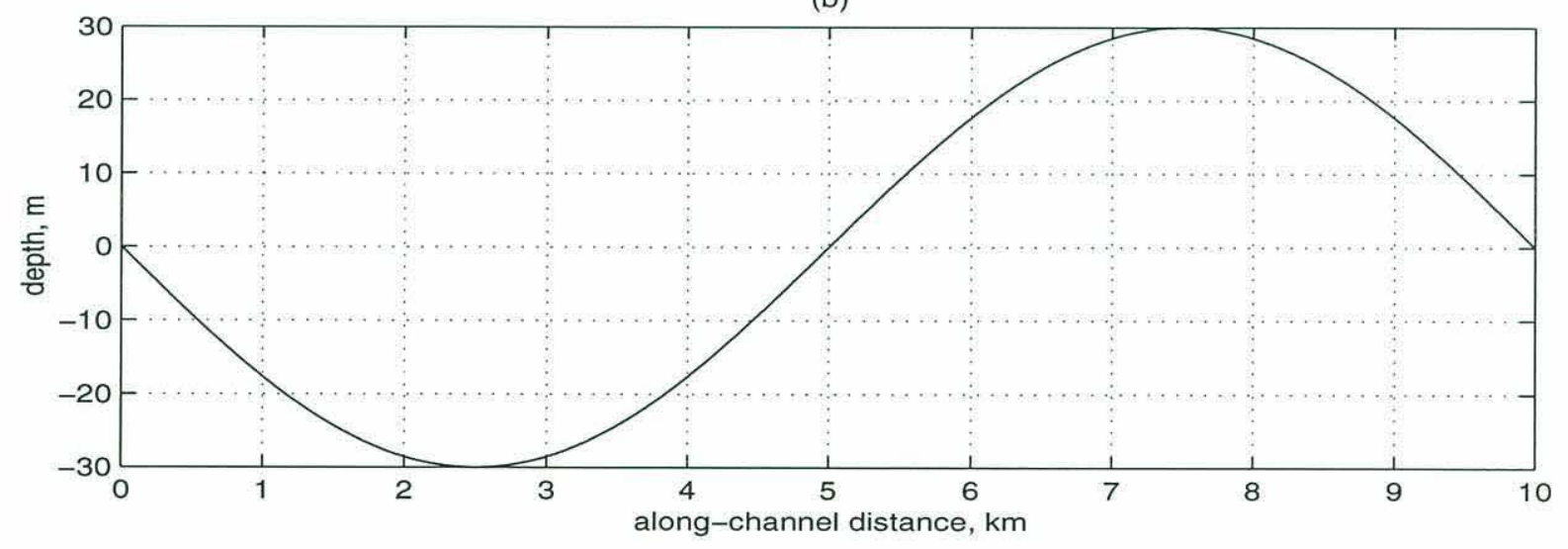

Figure 17: (a) The $t-x$ diagram of the cross-channel flow $(\mathrm{cm} / \mathrm{sec})$ from experiment 23 demonstrating short scale (relative vorticity) response; (b) a wavelength of topographic perturbation which is constructed from single sinusoid in this experiment. Both (a) and (b) taken from the mid-shelf section. 
(a)

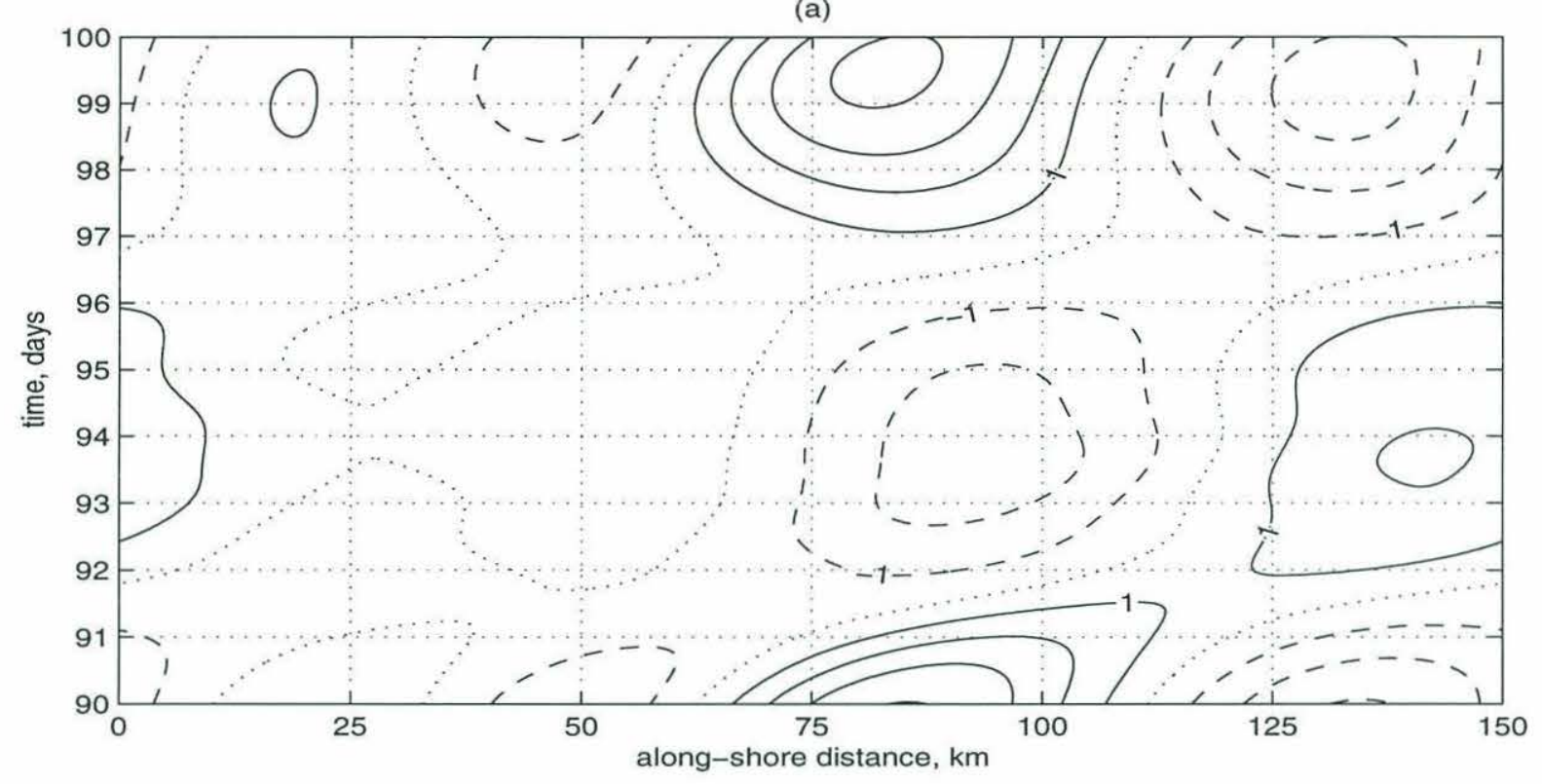

(b)

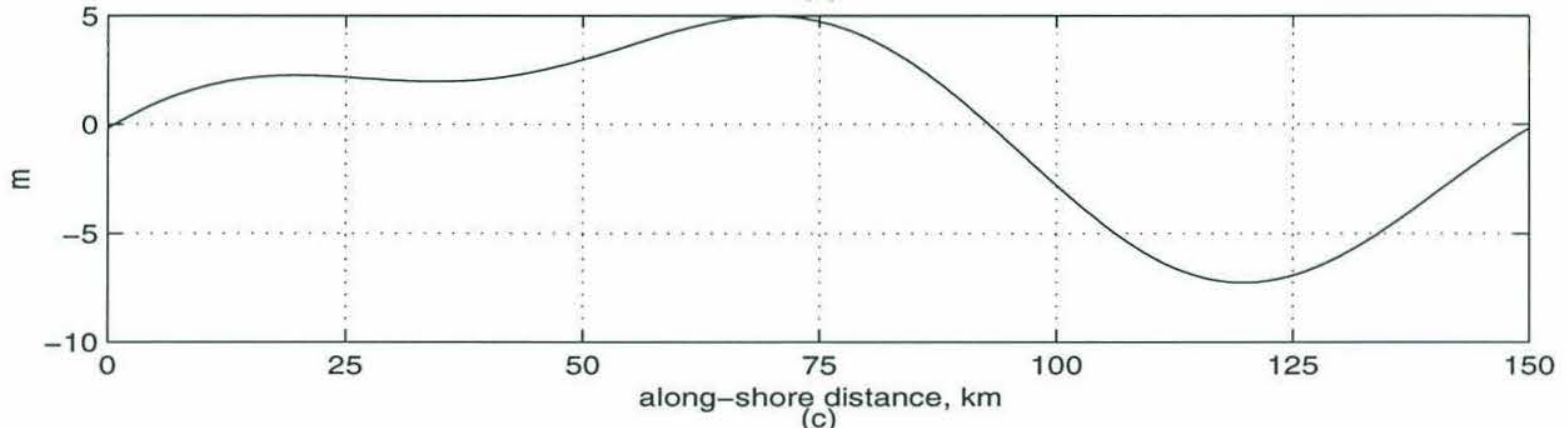

(c)

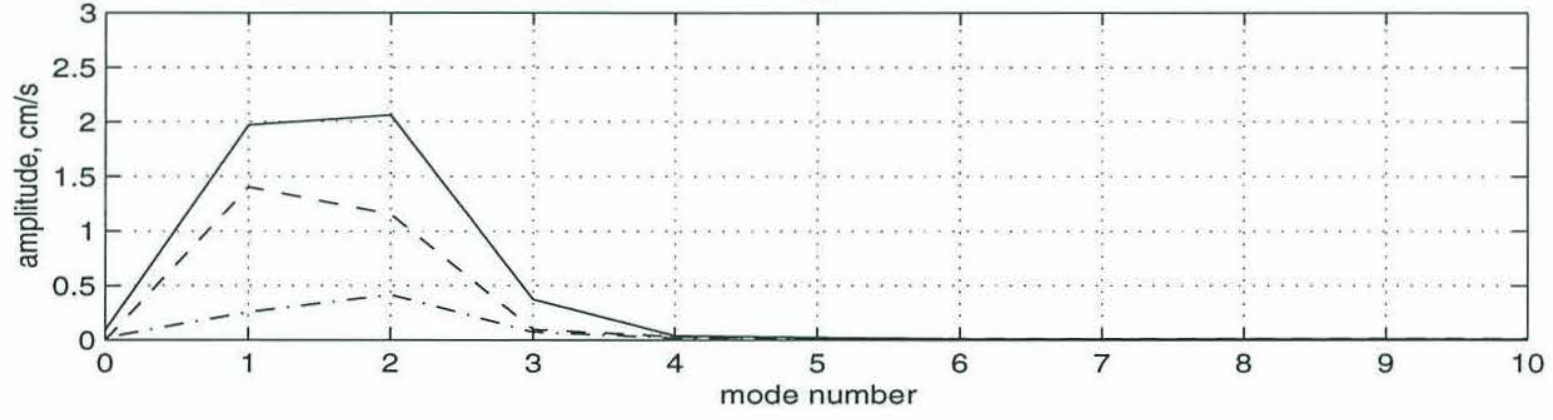

Figure 18: (a) the same as in Figure 17a but for experiment 21 (long wave response demonstration); (b) topographic perturbation due to a sum of mode 1 and 2; (c) amplitude of $v$ Fourier components averaged over $t=98-100$ days (lee wave phase) - solid line, $t=93-95$ days (no-lee wave phase) dashed line and over whole period - dash-dot line. 
(a)

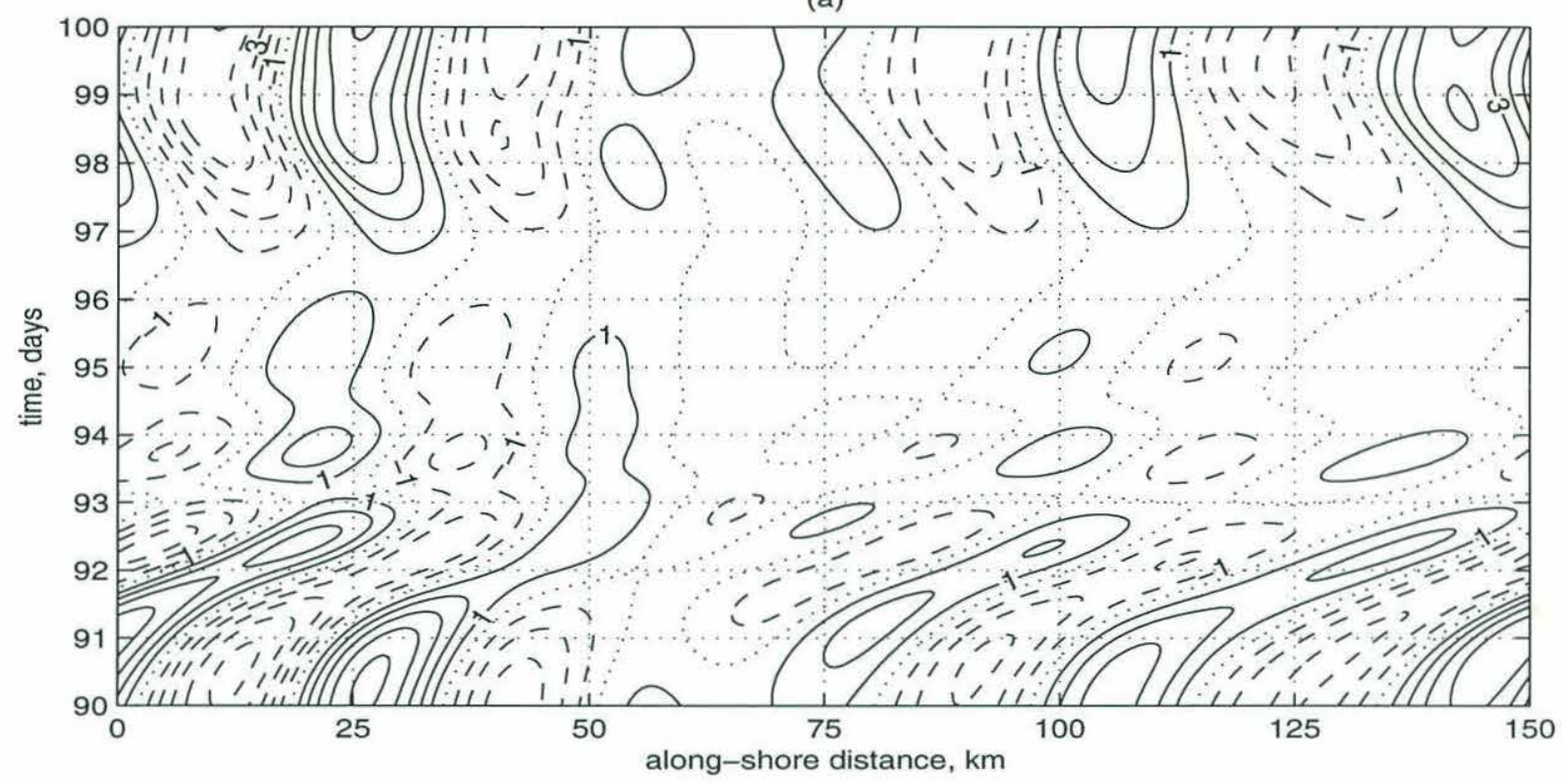

(b)

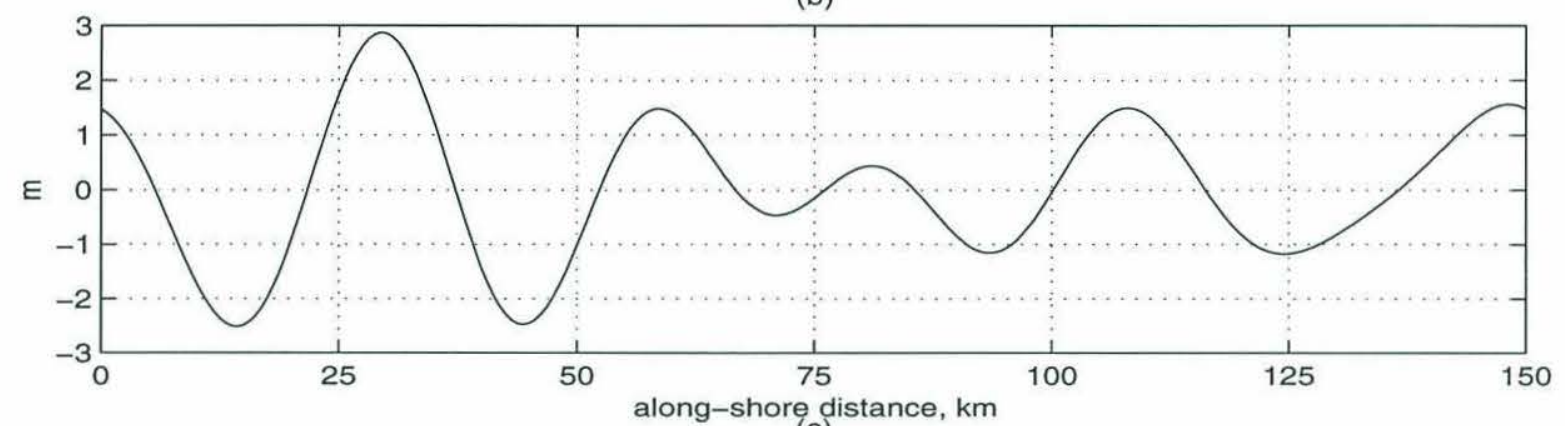

(c)

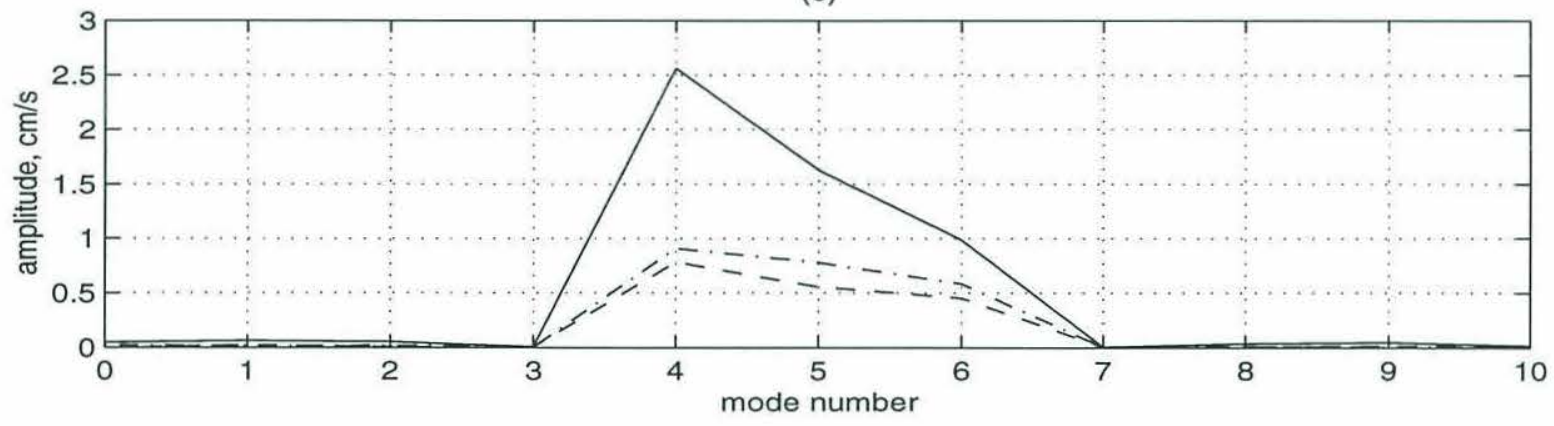

Figure 19: The same as in Figure 18 but for experiment 22 in which bottom bumps represented by the intermediate scales disturbances: modes 4,5 and 6 (alongshore wavelengths $37.5,30$ and $25 \mathrm{~km}$, respectively). 
(a)

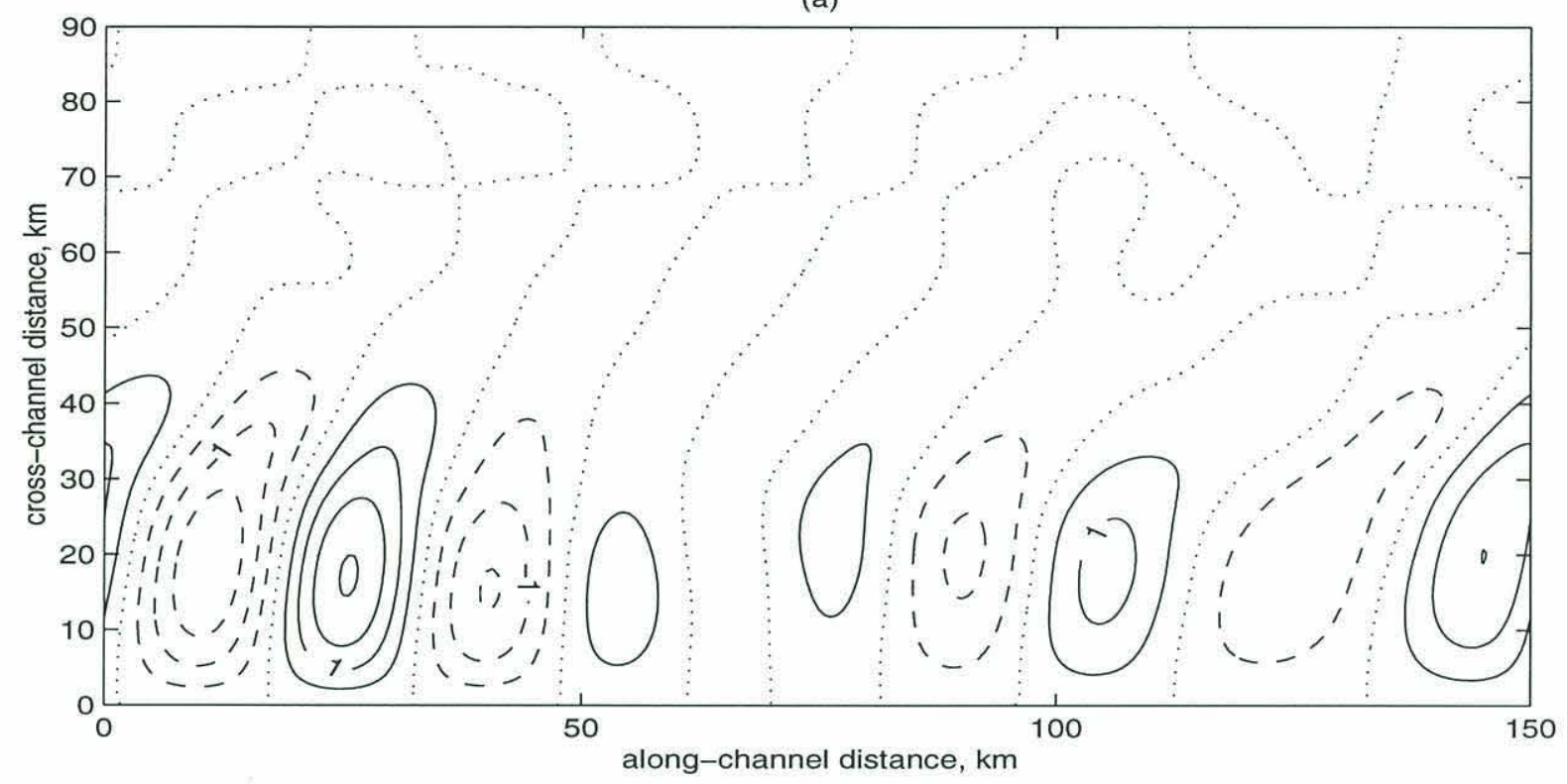

(b)

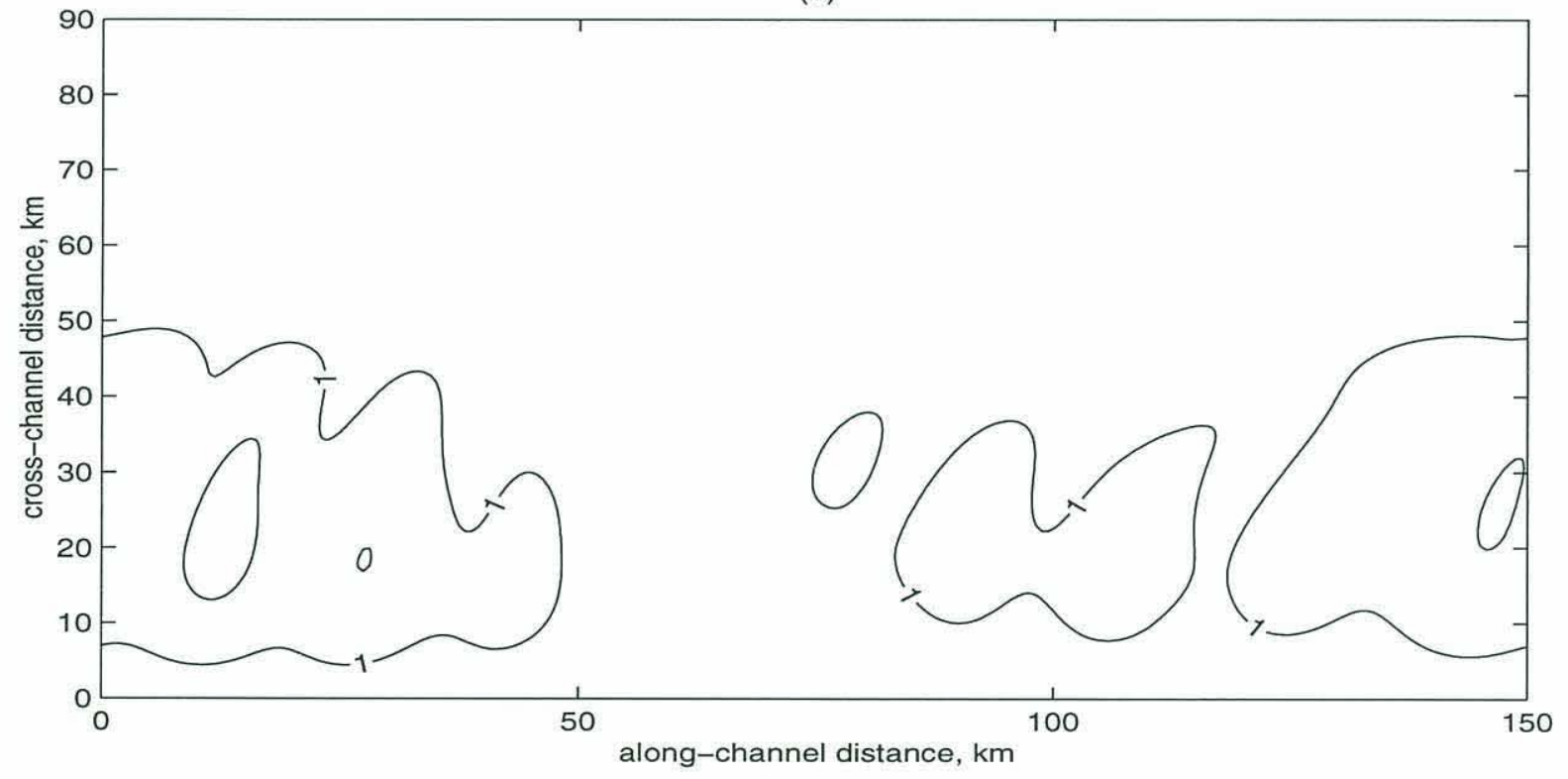

Figure 20: For experiment 22 (intermediate scale response): (a) time mean offshore currents $(\mathrm{cm} / \mathrm{sec}$ ); (b) standard deviation of cross-channel currents $(\mathrm{cm} / \mathrm{sec})$. 
(a)

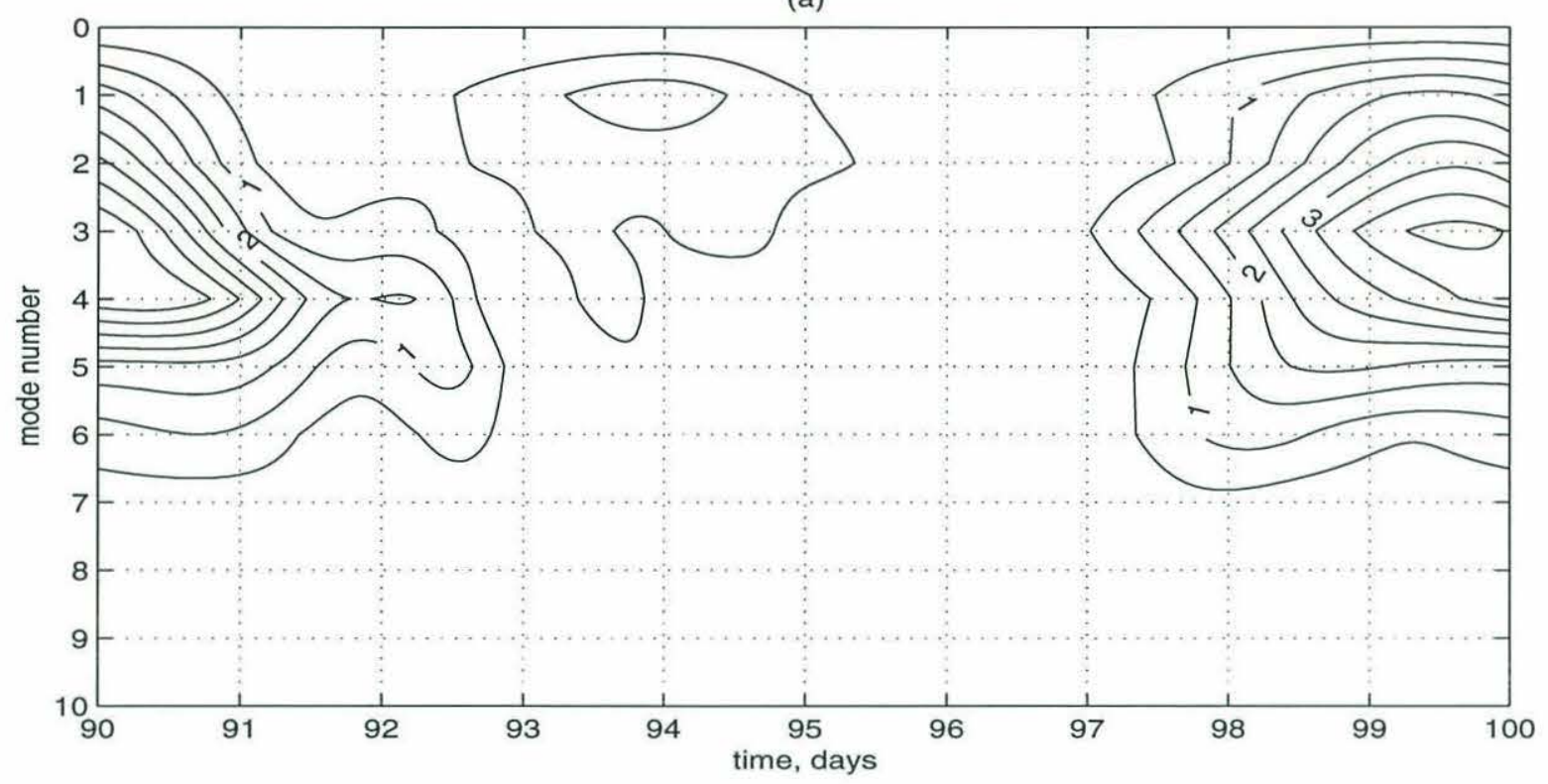

(b)
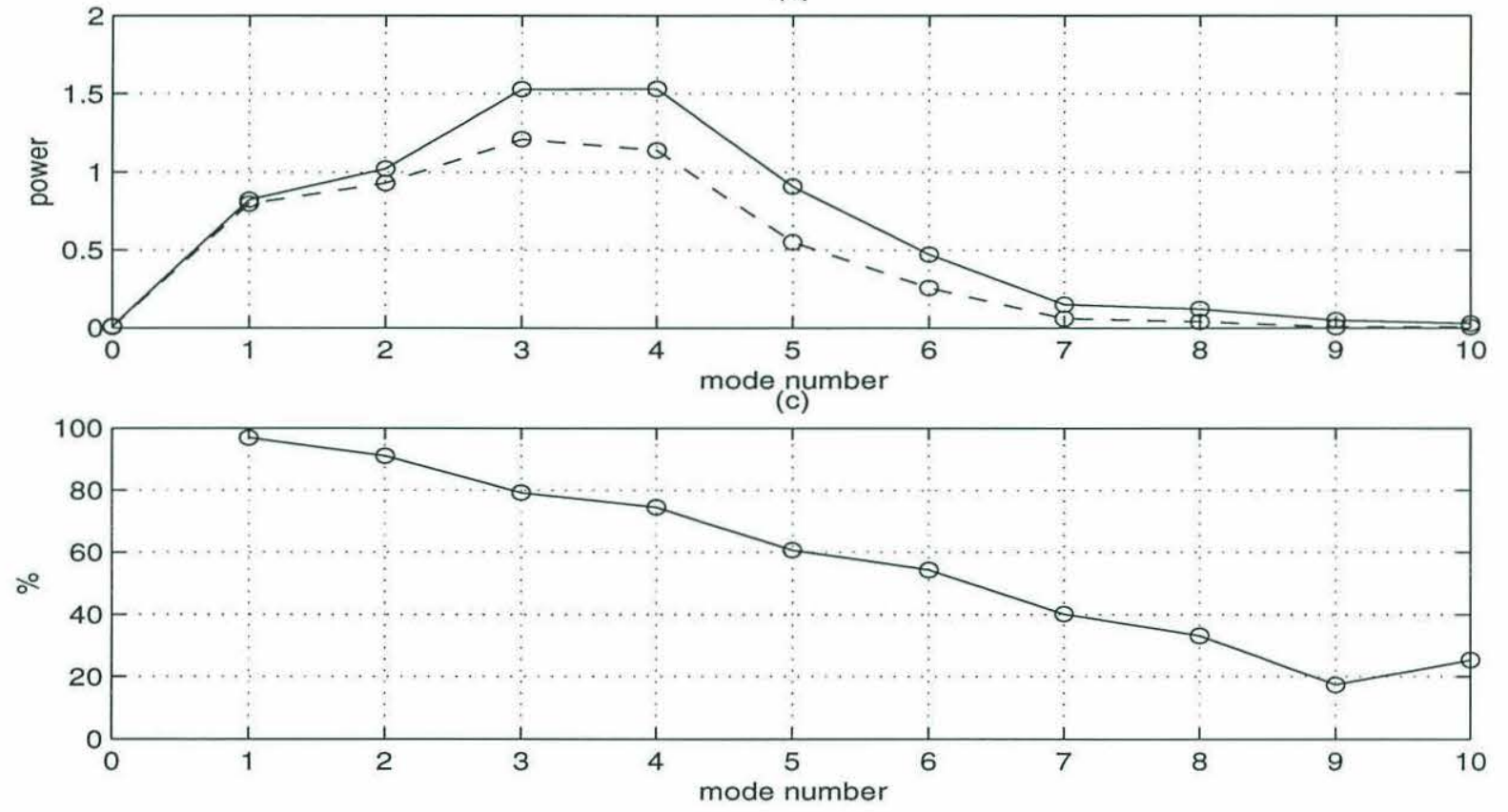

Figure 21: Basic experiment, mid-shelf section:(a) evolution of $v$ alongshore spectrum for a period; (b) mean (over a period) alongshore spectrum of the total cross-channel flow (solid line) and the fluctuating component (dashed line); (c) portion of the fluctuating energy component of total $v$ (mean over a period alongshore number spectrum). 


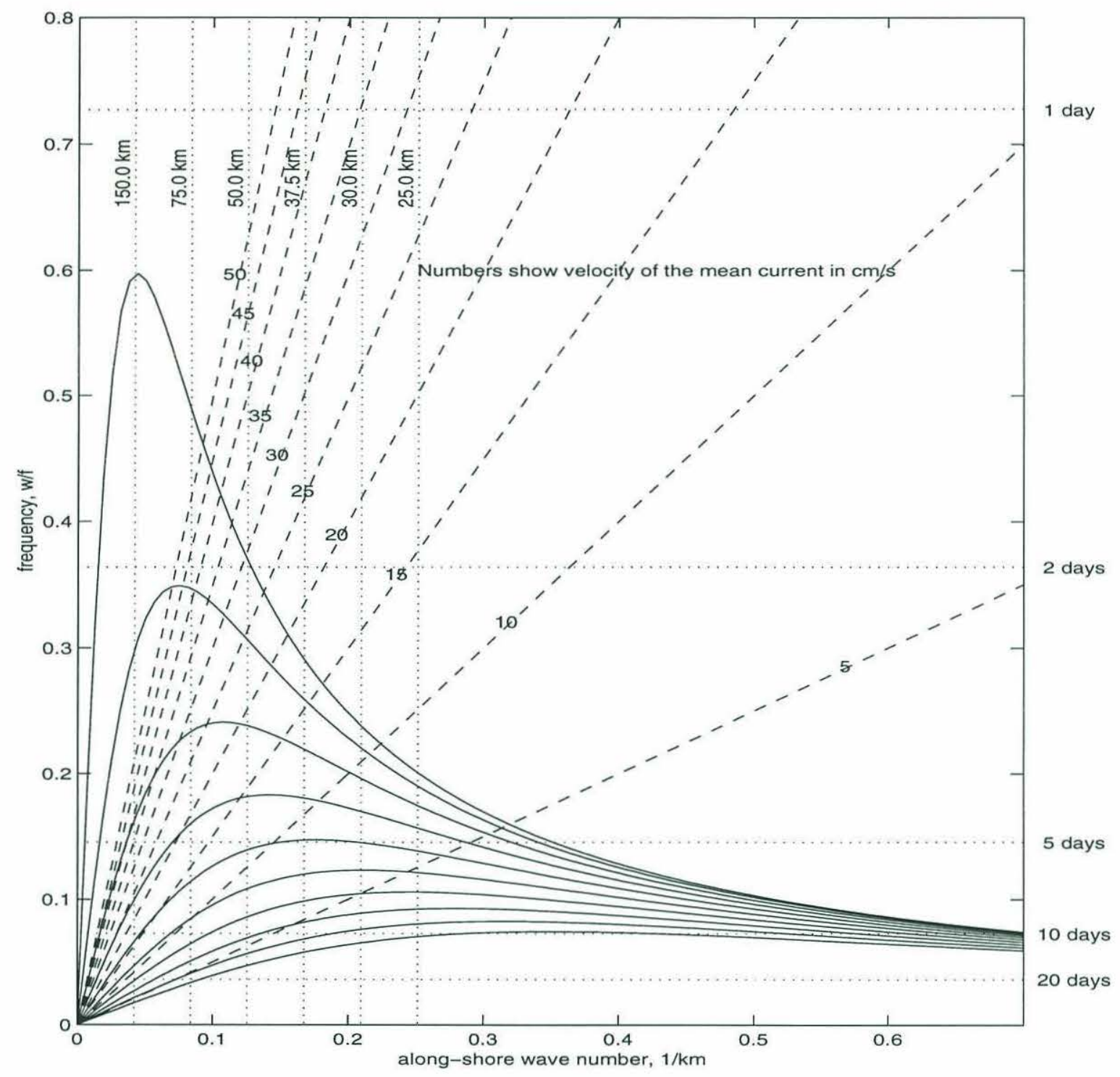

Figure 22: Barotropic Shelf Wave dispersion diagram for the background (exponential) topography. 
Figure 23. Dependence of the cross-channel flow characteristics on the wind amplitude (experiments 3,24 and 25): (a) standard deviation of the fluctuating component of $v$ as a function of crosschannel distance; (b) its ratio to the amplitude of the alongshore fluctuating component vs. $y$; (c) alongshore correlation distance ( $e$-folding scale of the $v^{t}$ autocorrelation function) at the mid-shelf section.

$\infty$

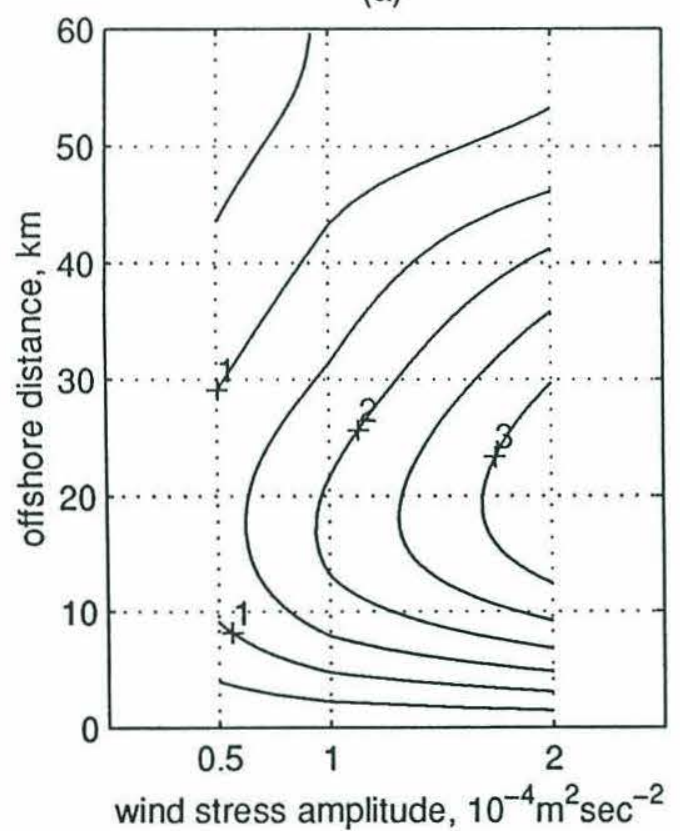

(b)

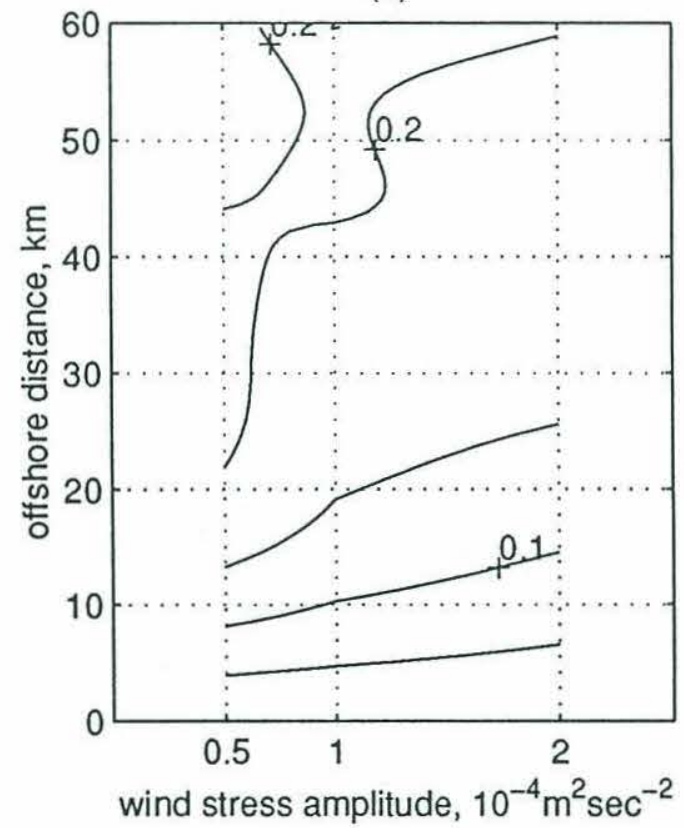

(c)

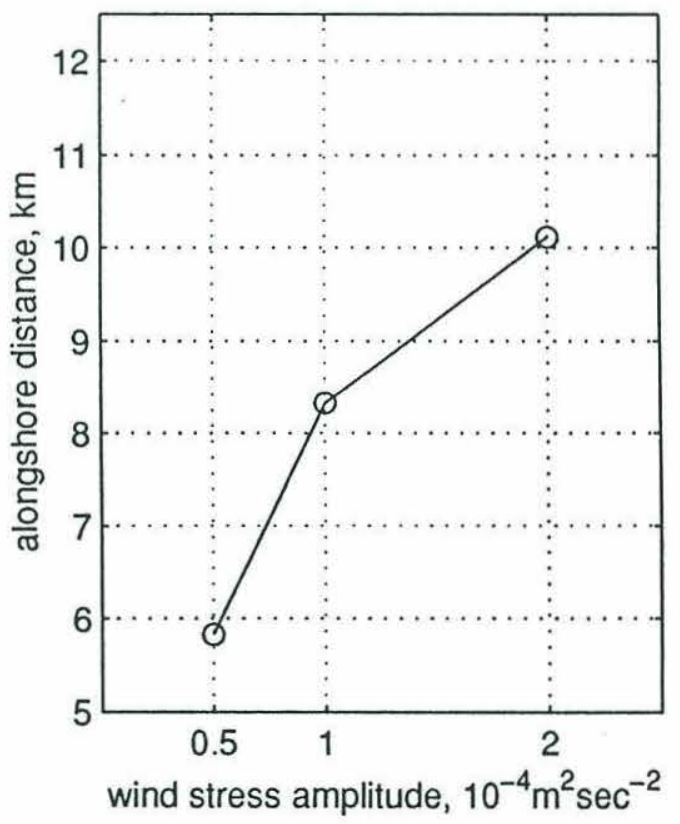


Figure 24. The same as in Figure 23 but for experiments 1-5: dependence on the wind period.

8

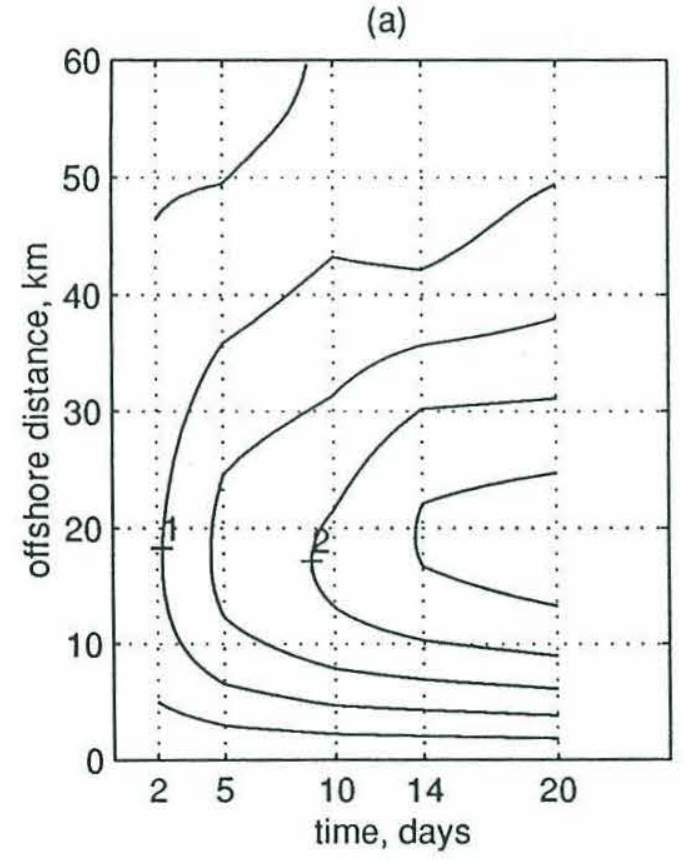

(b)

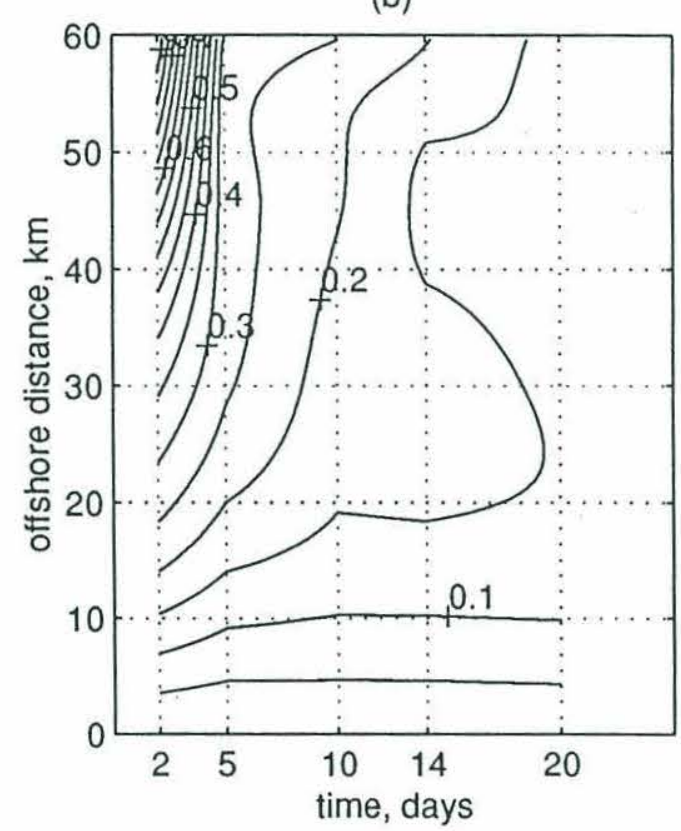

(c)

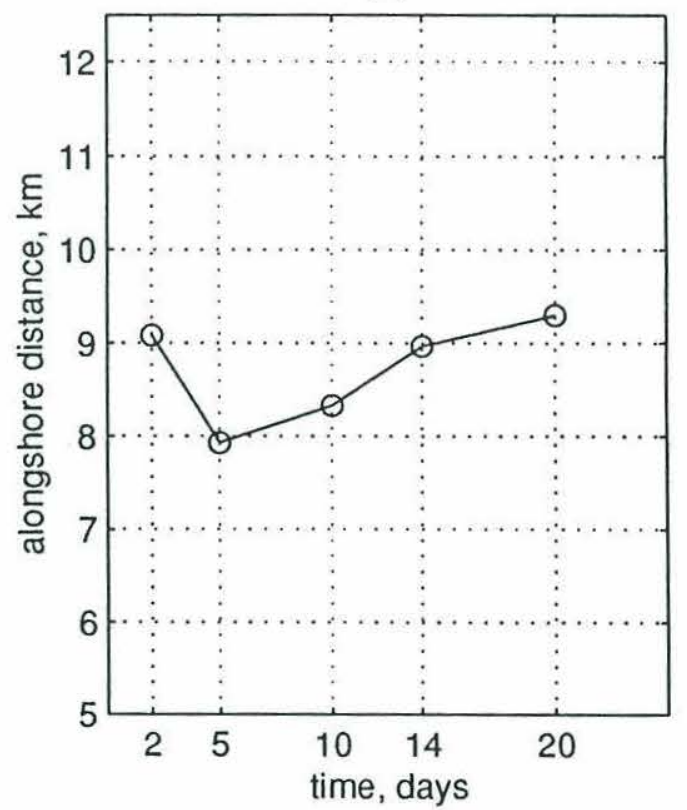


Figure 25. The same as in Figure 23 but for experiments 16-20: dependence on friction.

$\bullet$

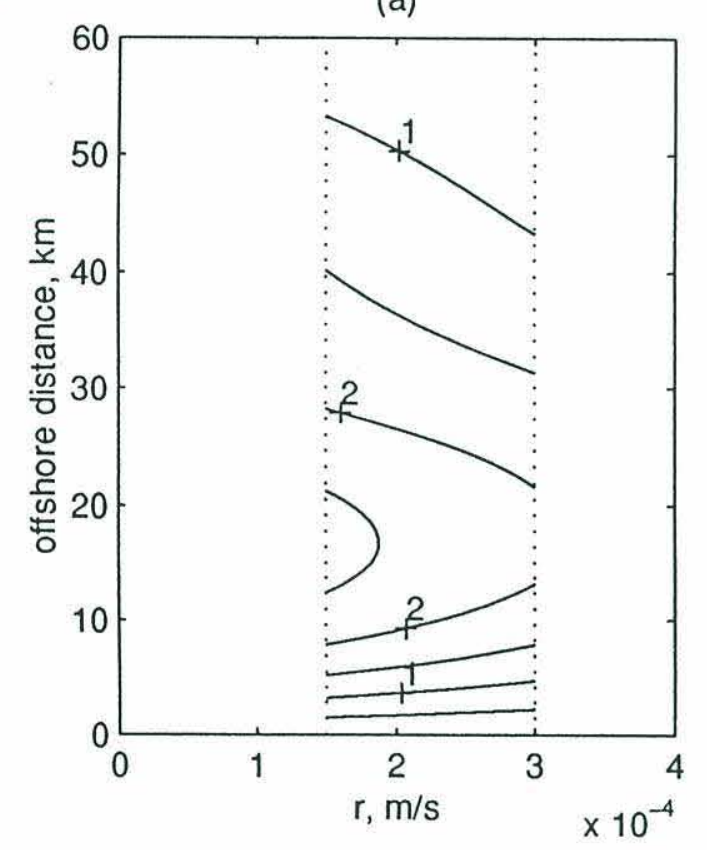

(b)

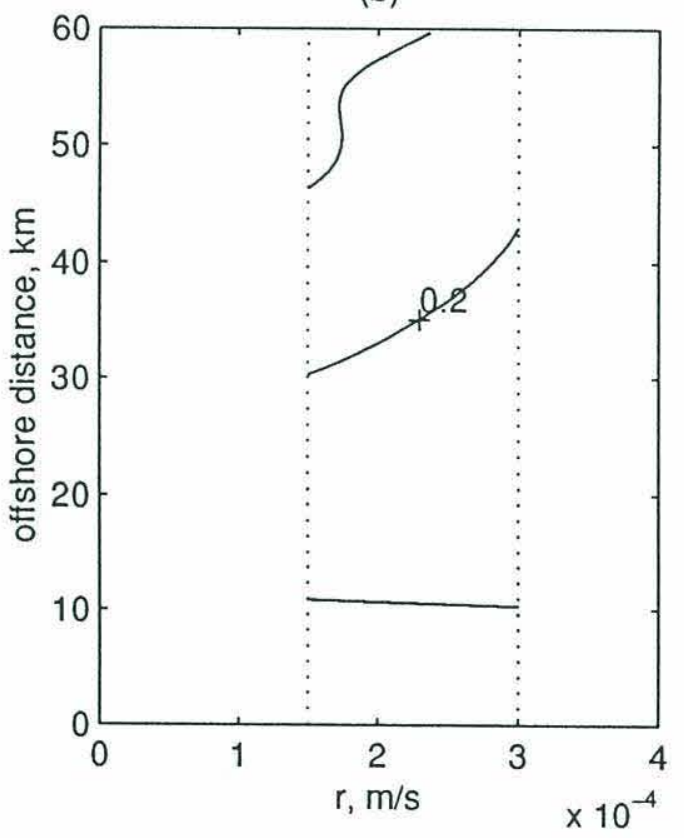

(c)

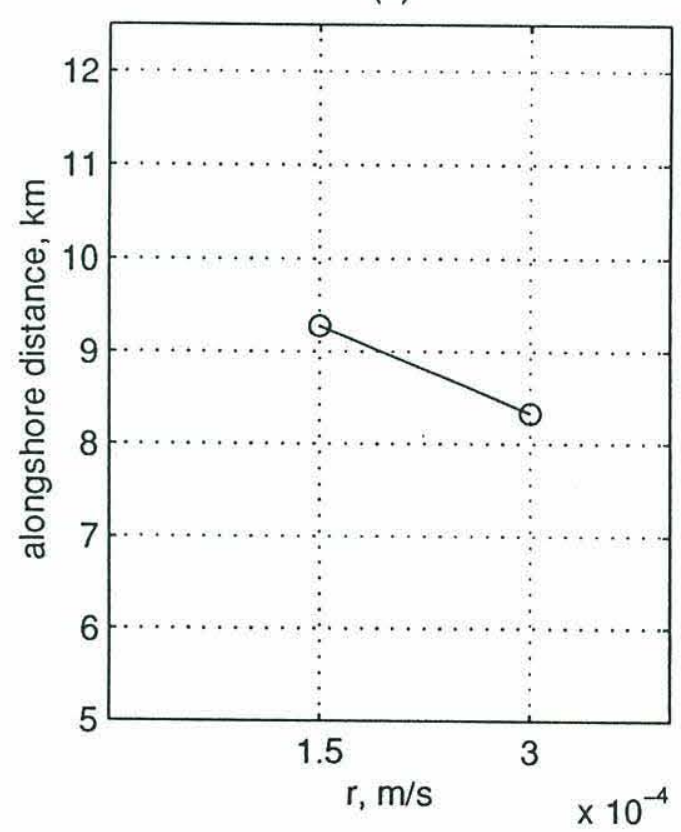


Figure 26. The same as in Figure 23 but for experiments 3,8 and 13: dependence on the bump amplitude.

$\stackrel{0}{\circ}$

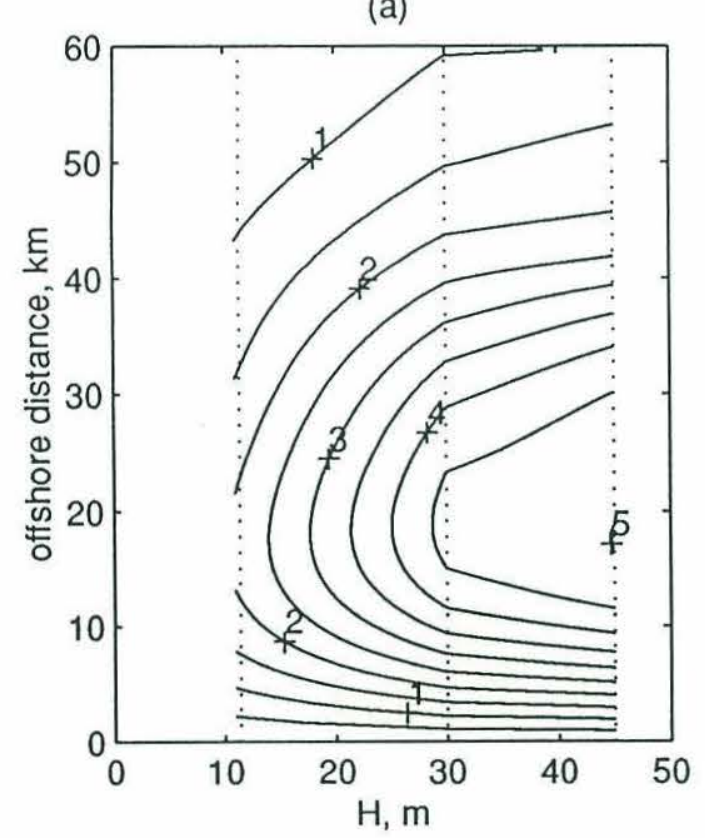

(b)

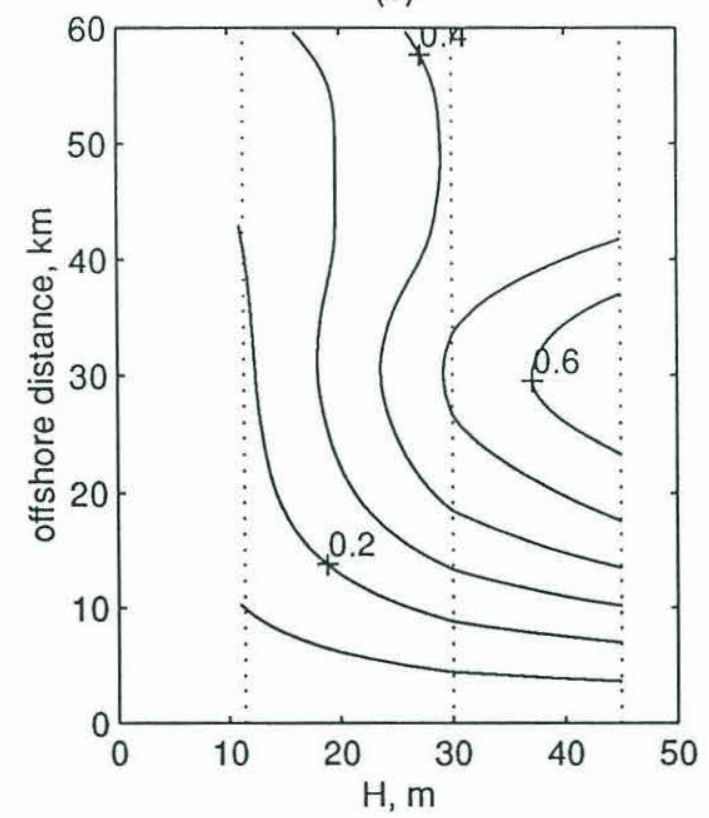

(c)

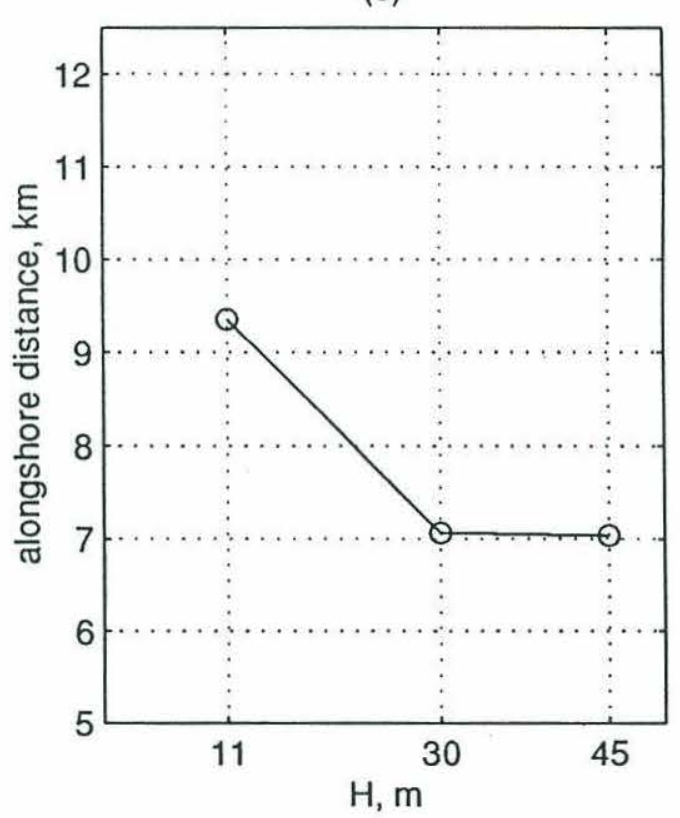


Figure 27. The same as in Figure 23 but for experiments 3 and 26: sensitivity to the bump spectral shape. On the $x$-axis 2 corresponds to topography with $k_{T}^{-2}$ spectrum, while 3 to $k_{T}^{-3}$ spectrum.

$\mathscr{\omega}$

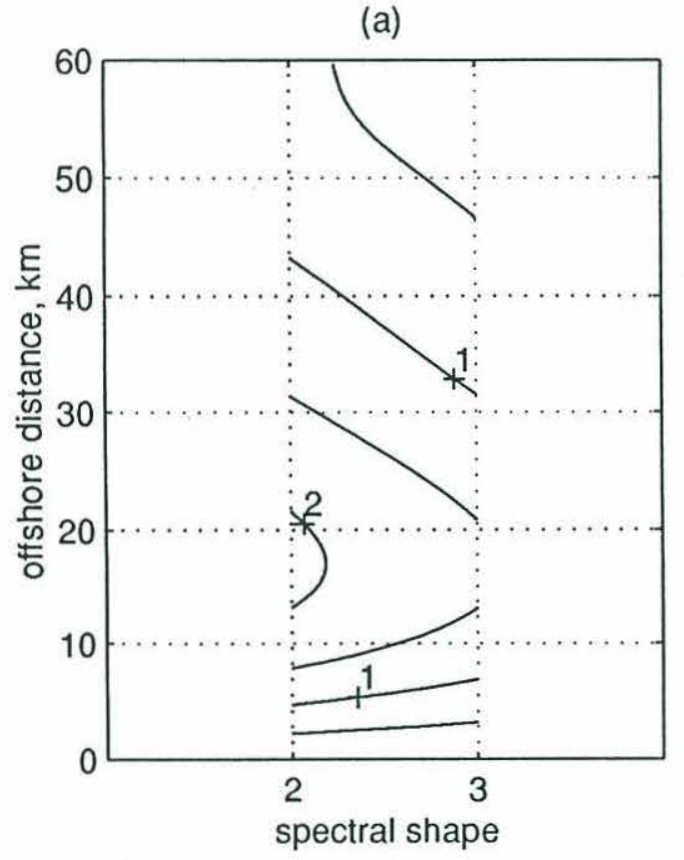

(b)

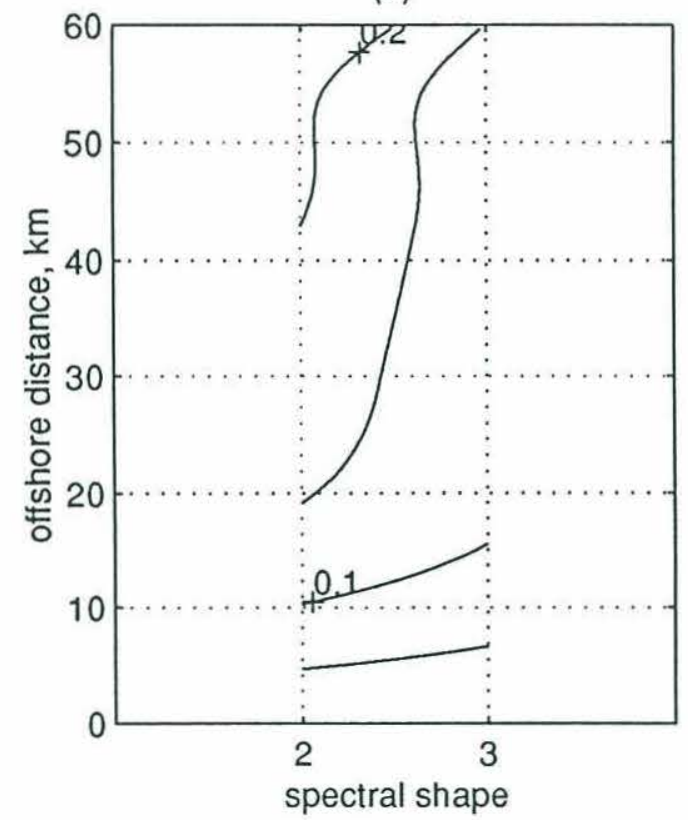

(c)

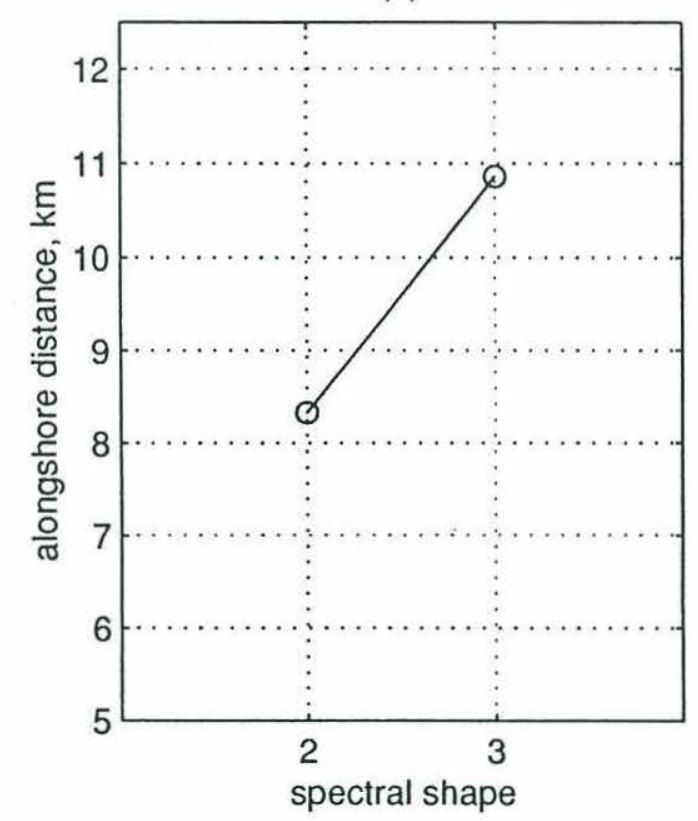


Figure 28. The same as in Figure 23 but for experiments 4 (14 days wind period) and 27 (two-frequency wind: 4 and 14 days period). On the $x$-axis 1 corresponds to experiment $4 ; 2$ - to experiment 27 .

$\stackrel{\wp}{\Perp}$

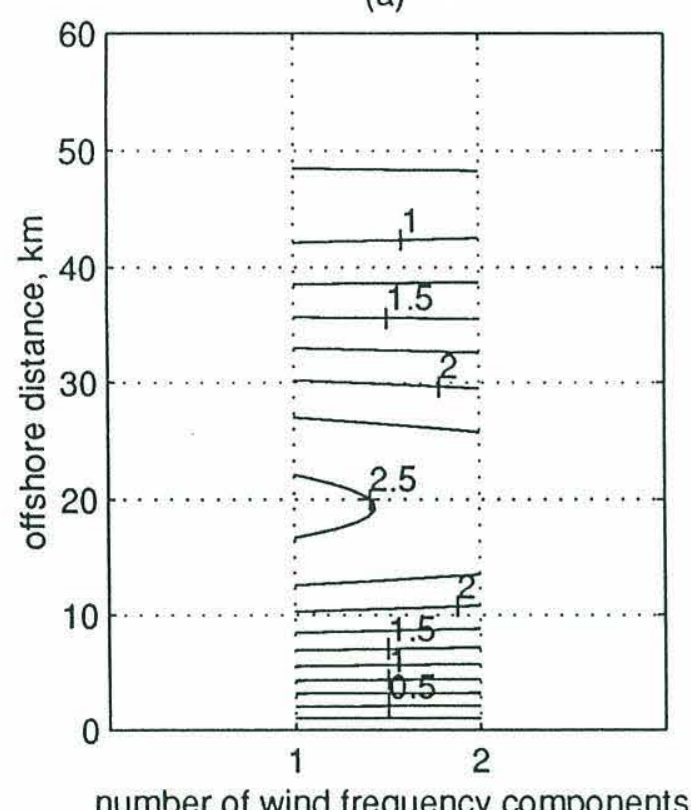

(b)

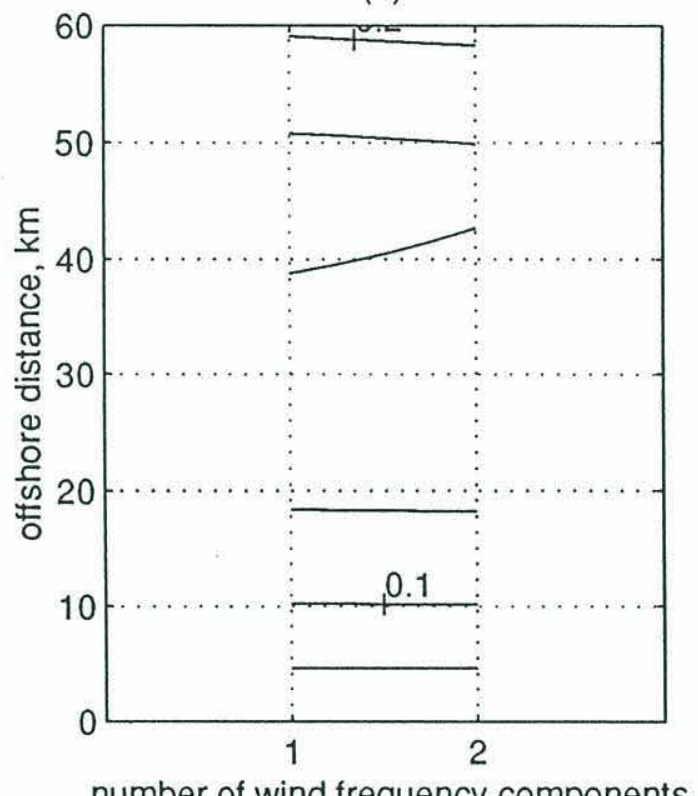

(c)

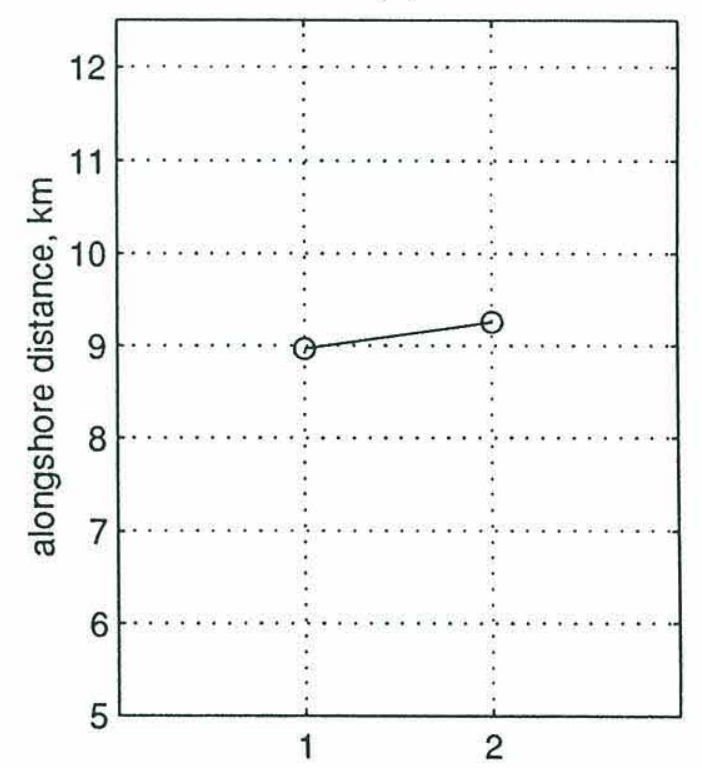

number of wind frequency components 
(a)

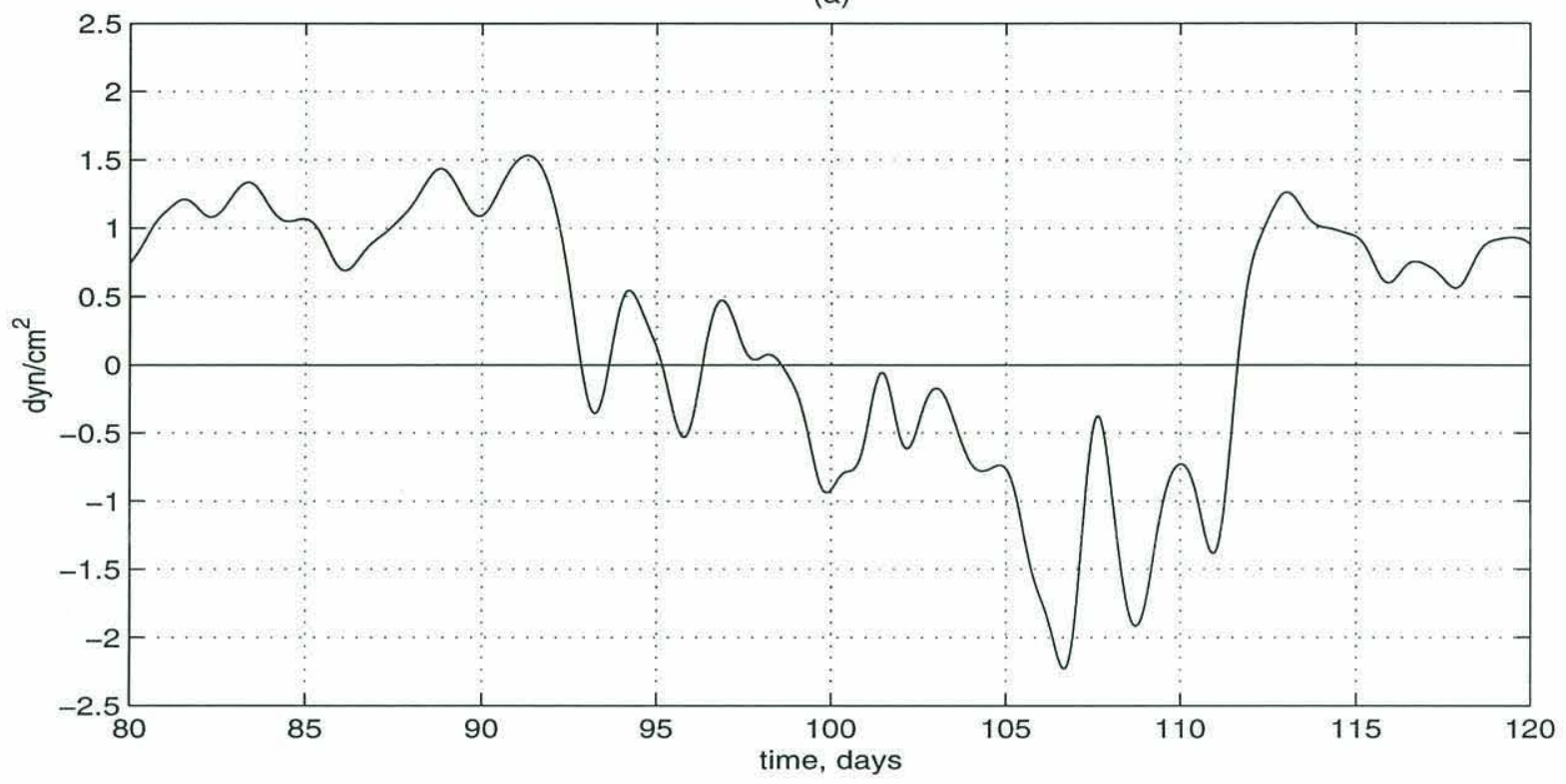

(b)

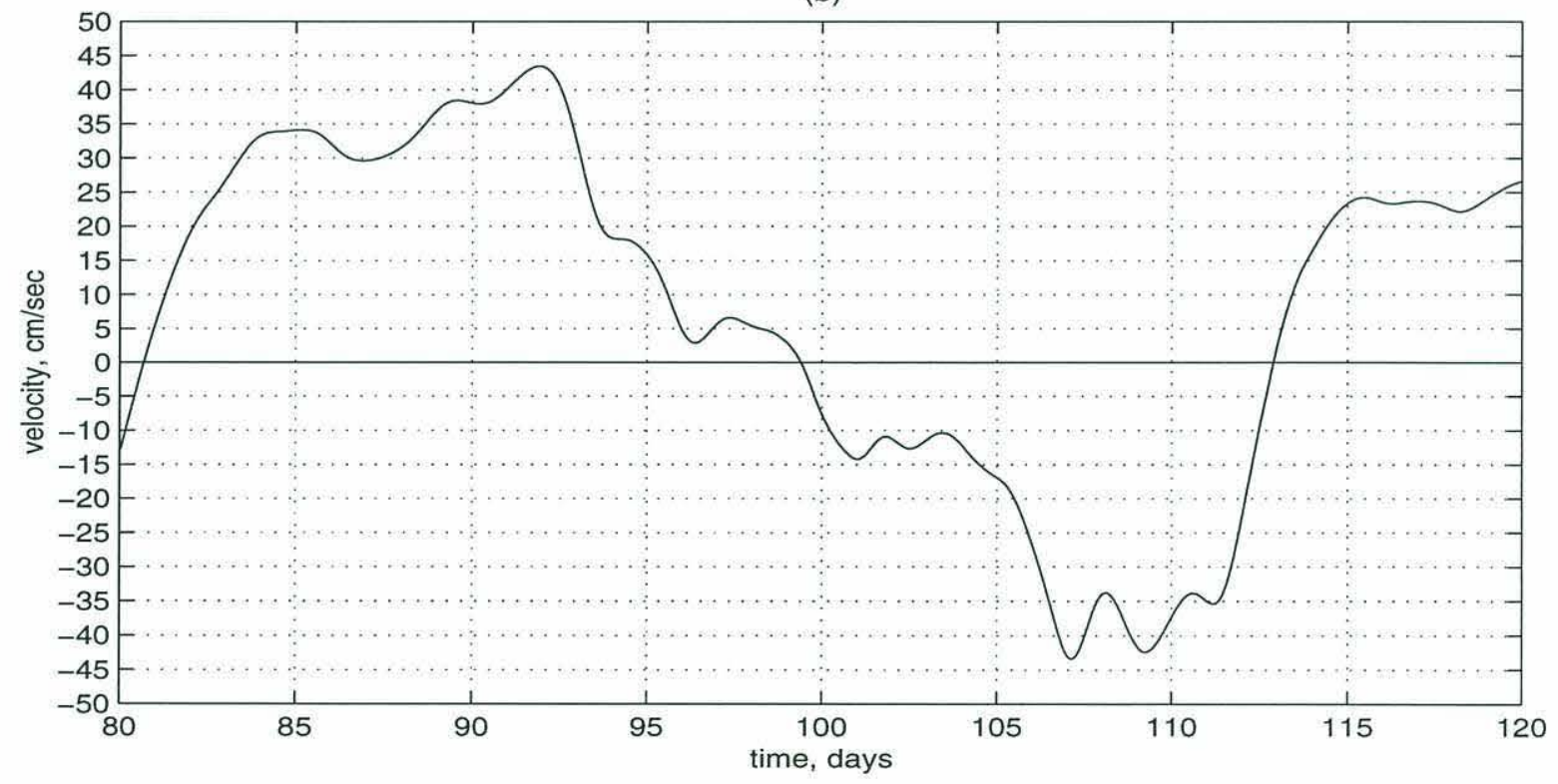

Figure 29: (a) last 40 days of CODE-2 low-frequency wind stress data used in experiment 28; (b) $x$-averaged alongshore flow resulted from the forcing by the CODE-2 wind. 
Figure 30. The same as in Figure 23 but for experiments 25 and 28 . On the $x$-axis 1 corresponds to experiment 25 (10 days wind period with $2 \cdot 10^{-4} \mathrm{~m}^{2} / \mathrm{sec}^{2}$ to experiment $4 ; 2$ - to experiment 28 (CODE-2 wind data).

8

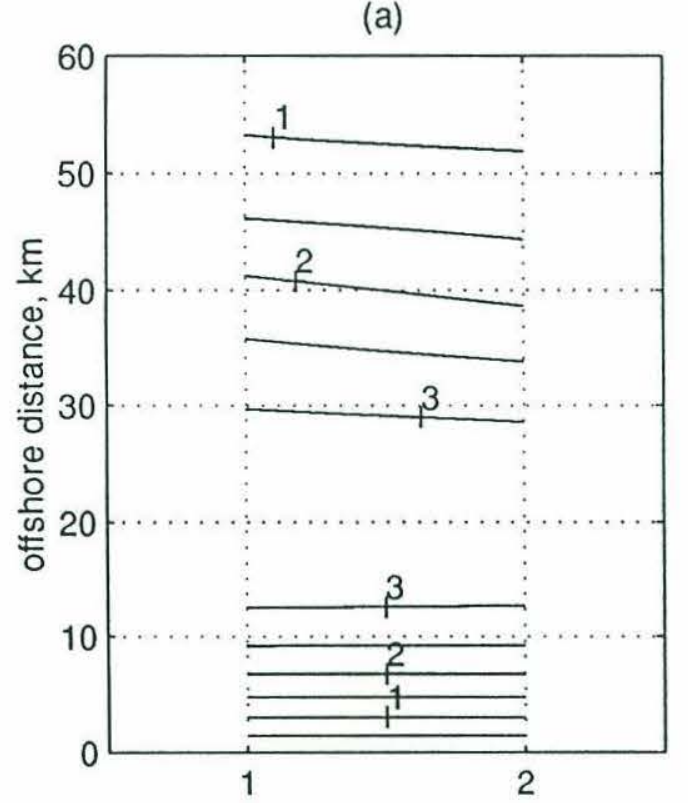

(b)

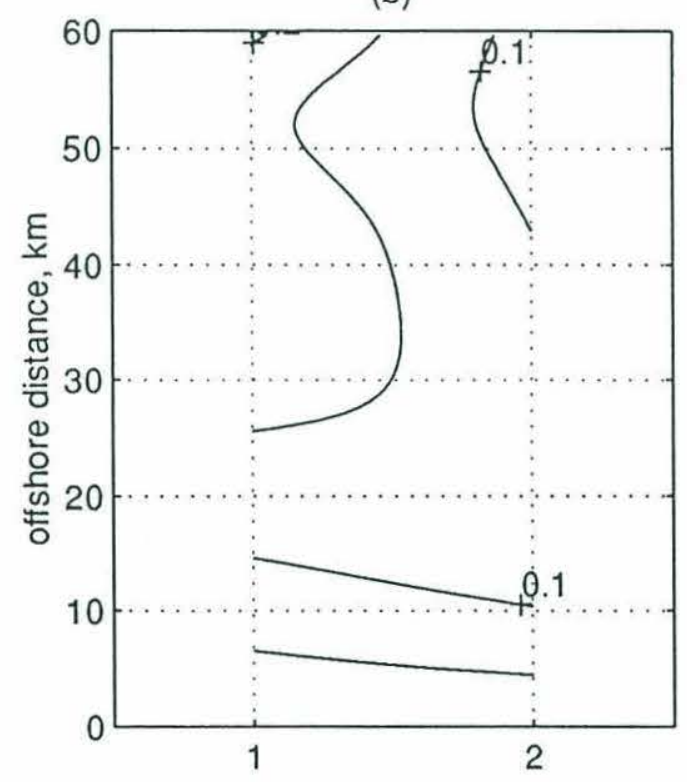

(c)

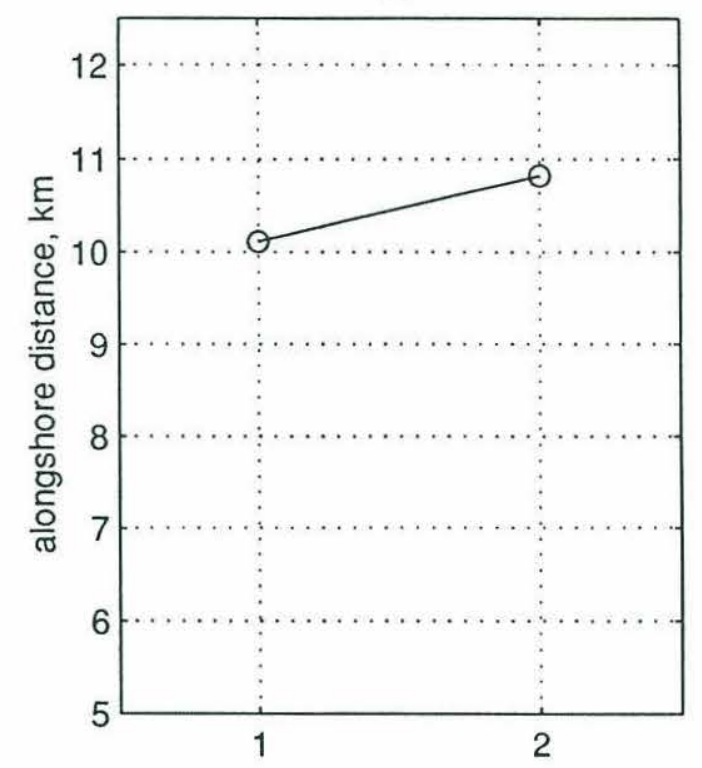


(a)

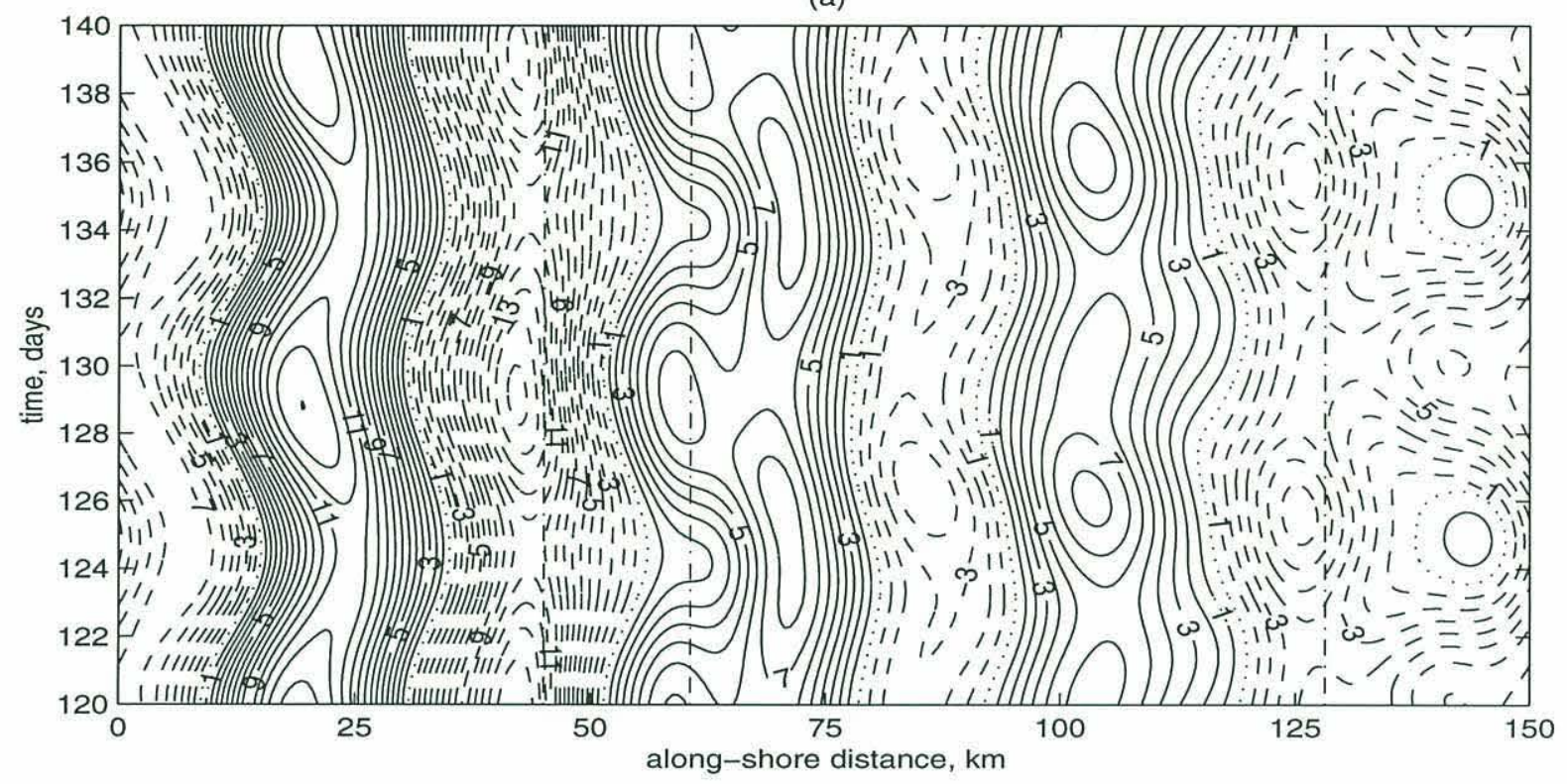

(b)

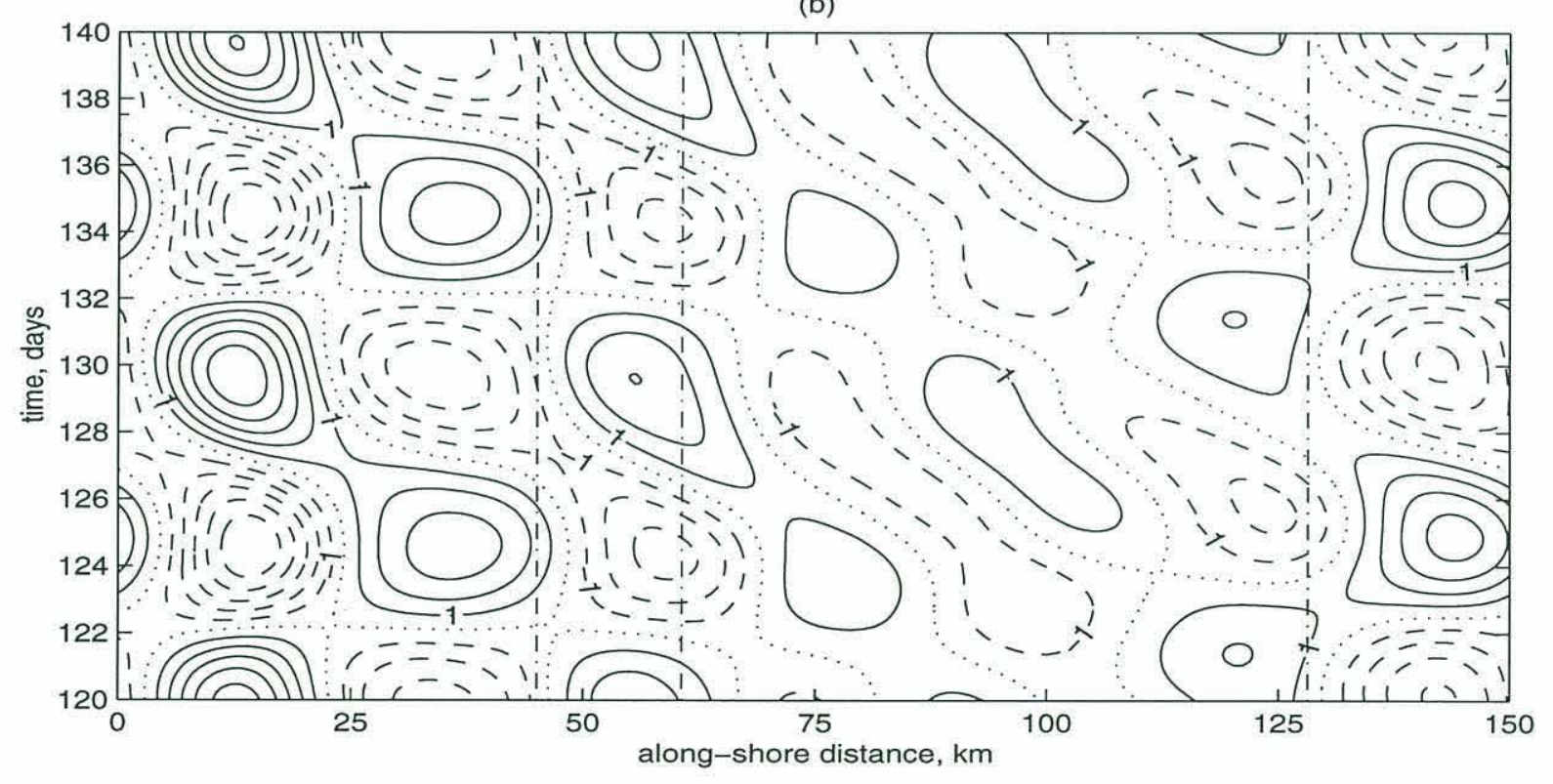

Figure 31: The $t-x$ diagram for (a) the total cross-shelf flow; (b) its fluctuating component. Experiment 29: $75 \%$ of wind energy is supplied by the negative mean wind stress, $25 \%$ - by the wind with 10 days period. 


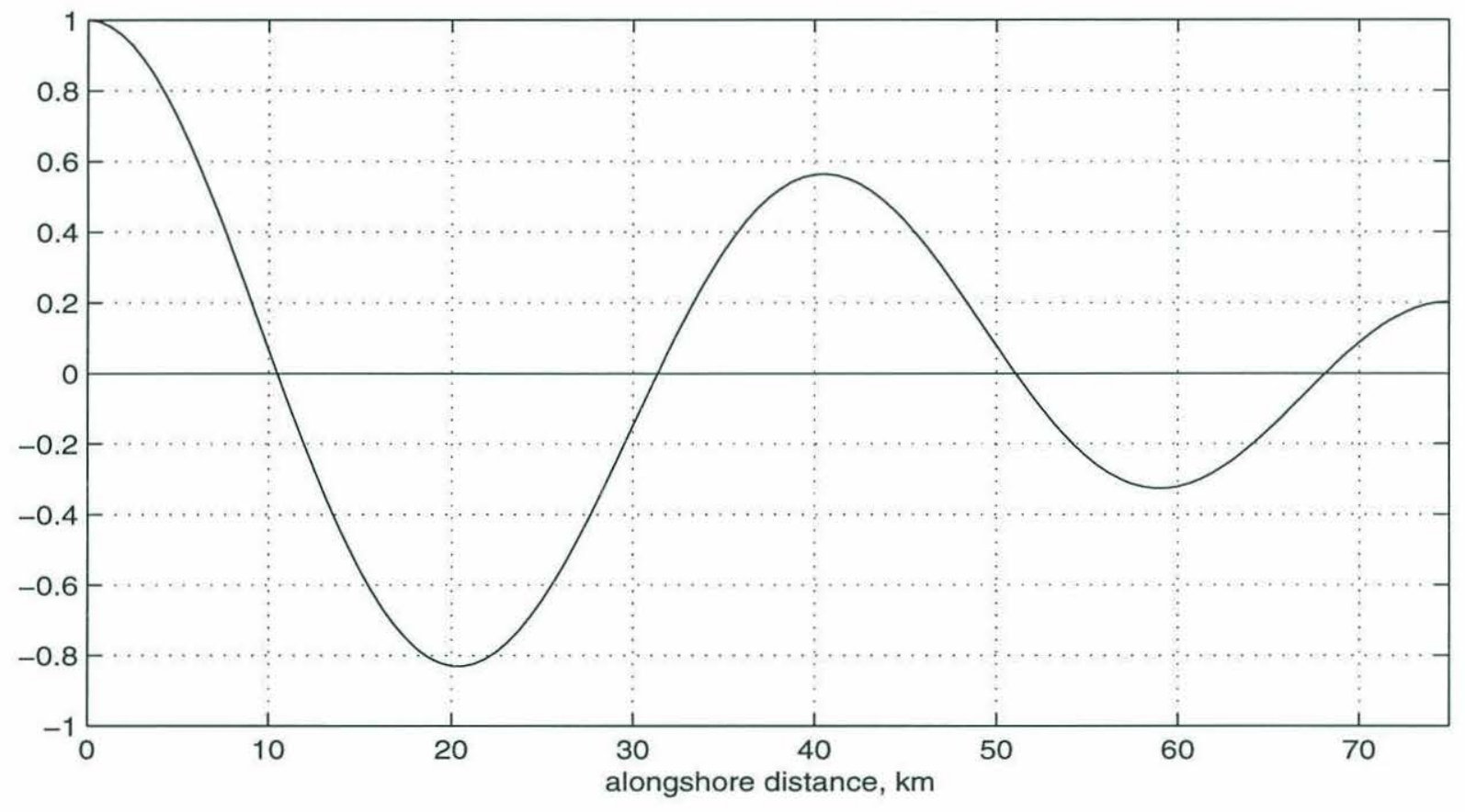

Figure 32: Autocorrelation function of the fluctuating component of $v$ for experiment 29. There is a well-determined maximum at $x \approx 40.6 \mathrm{~km}$ corresponding to the most energetic scale of $v$. 


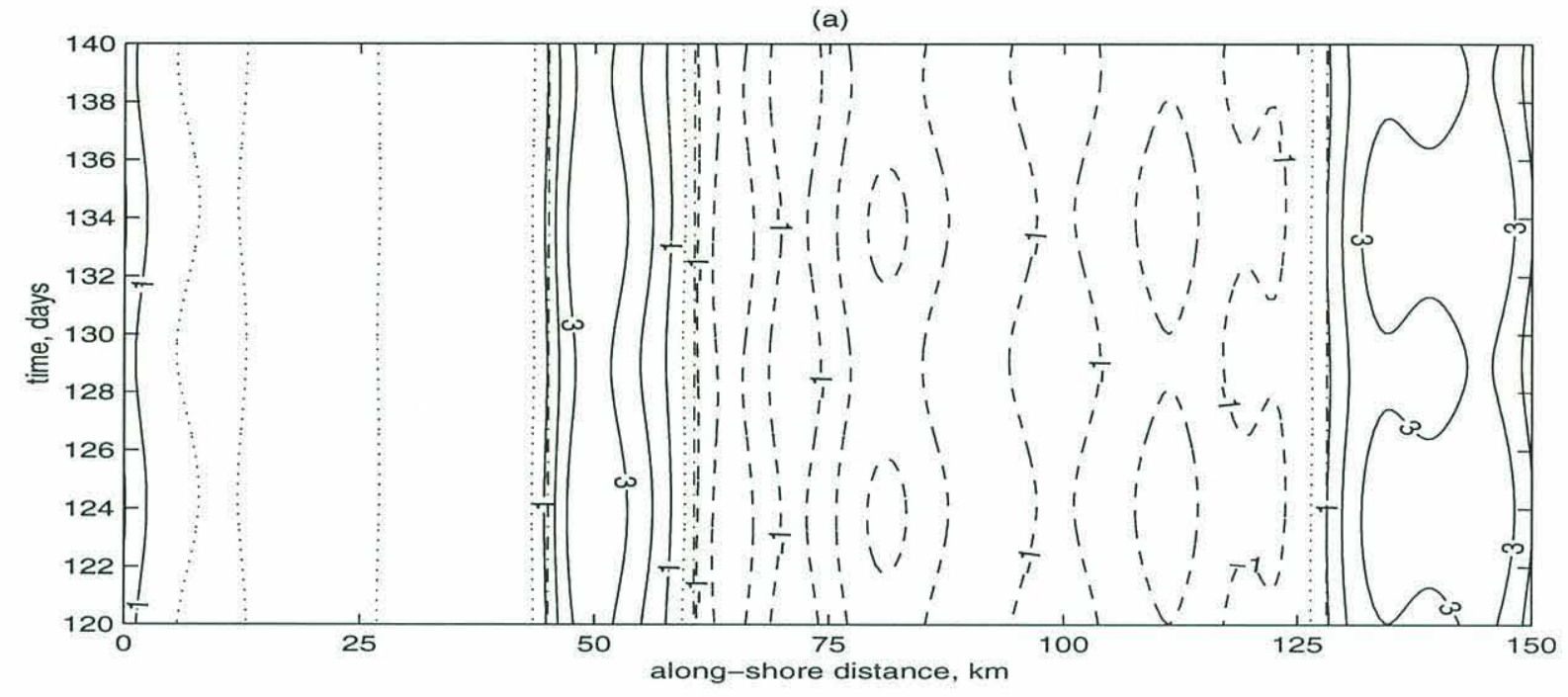

(b)

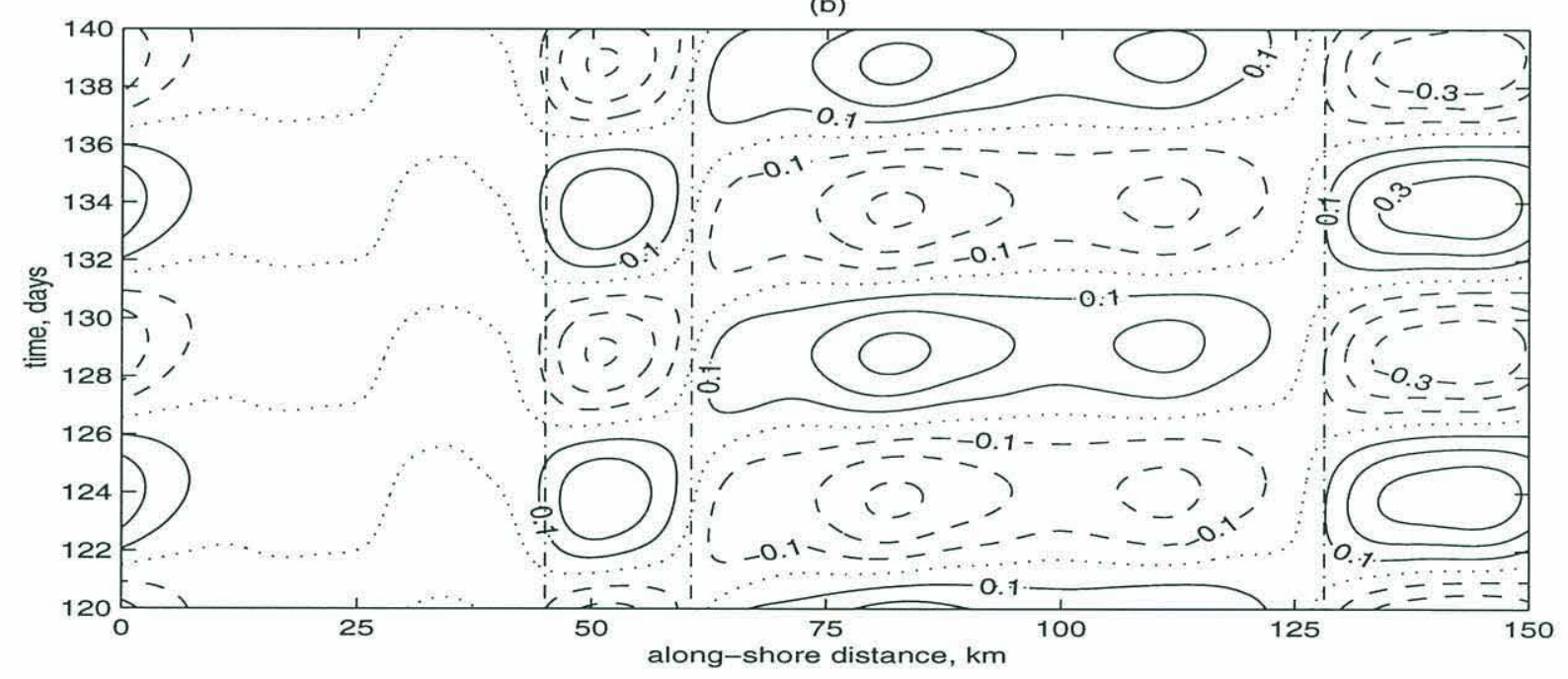

Figure 33: The same as in Figure 31 but for experiments 30 with positive mean wind. 


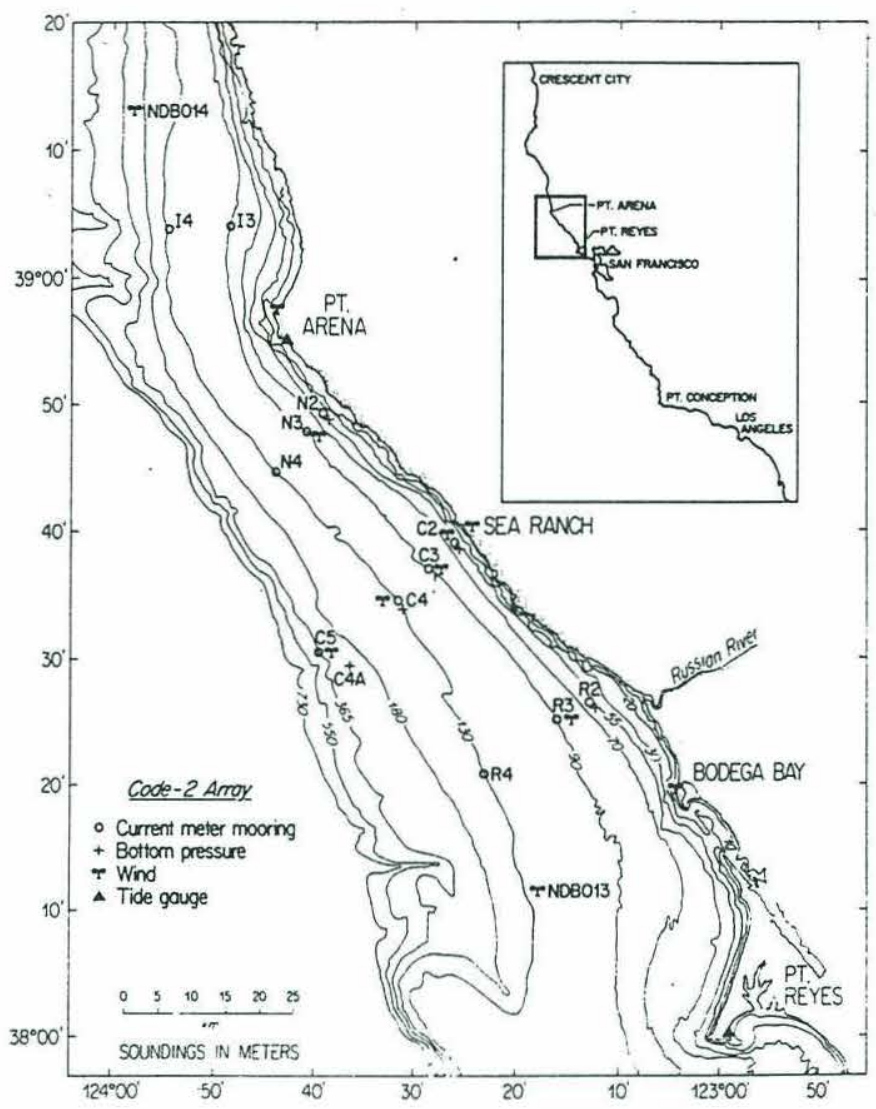

Figure 34. Map of the CODE region. (From Lentz, 1987.) 


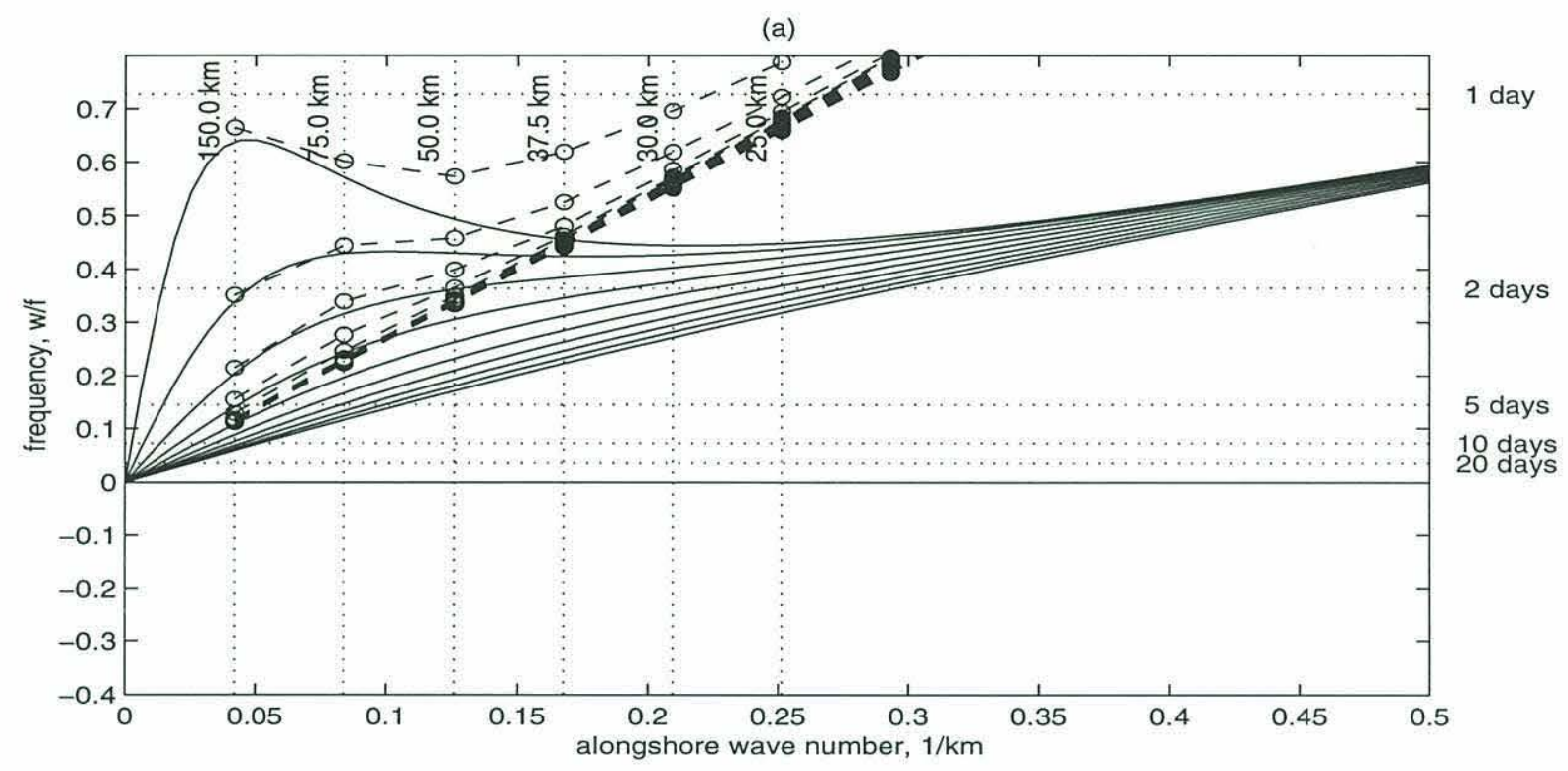

(b)

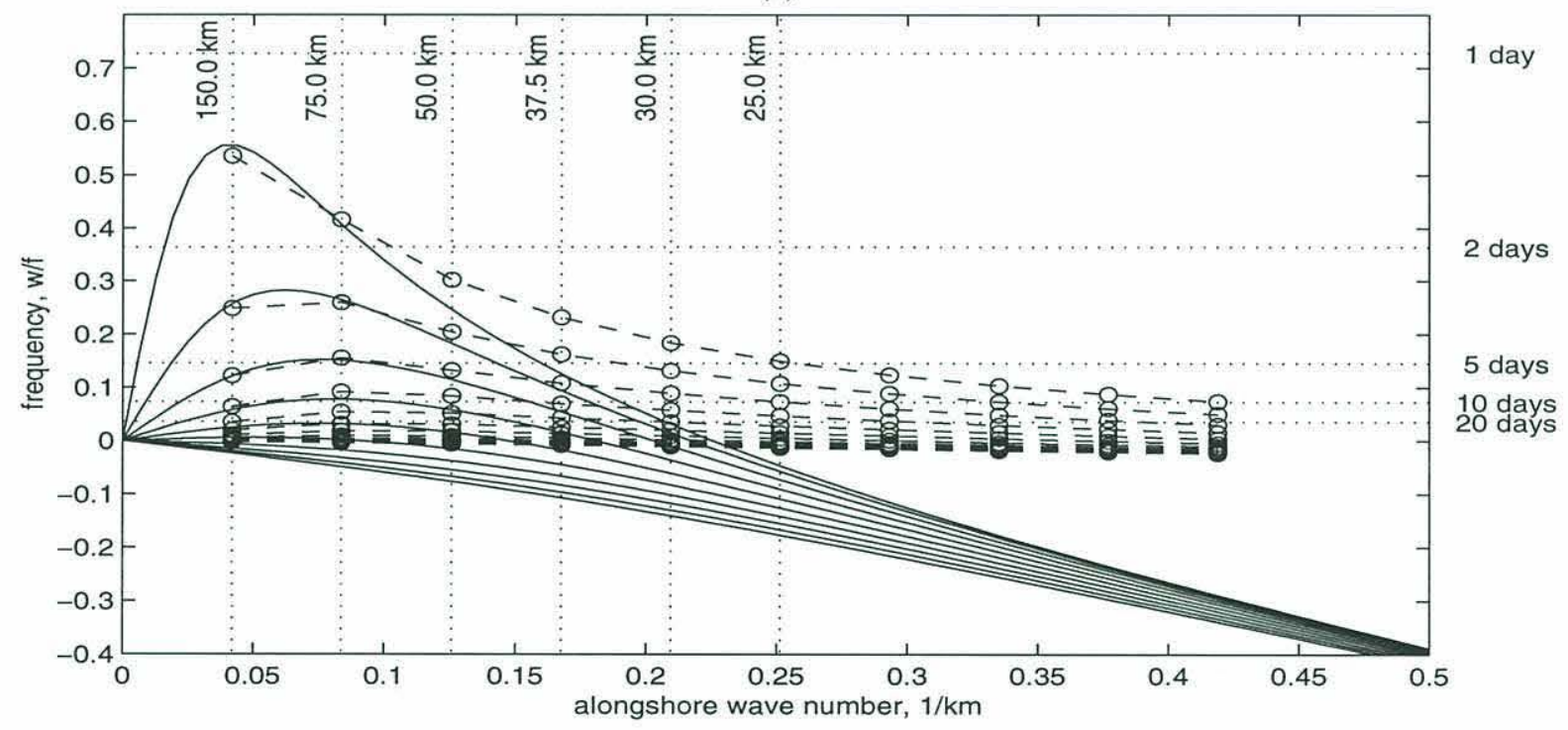

Figure A.1: BSW dispersion diagrams for the sheared background flow determined by equation (3.4), dashed lines with circles, and for the uniform (averaged in $y$ value of $U$ at some particular time) across the channel flow, solid lines: (a) the background flow profile and its $y$-average value are taken at $t=93.9$ days when $\bar{U}^{y}=9.8 \mathrm{~cm} / \mathrm{sec}$ (maximum, no-lee wave phase); (b) (a) the background flow profile and its $y$ average value are taken at $t=98.9$ days when $\bar{U}^{y}=-9.8 \mathrm{~cm} / \mathrm{sec}$ (minimum, lee wave phase). 